\title{
Role of Midline Thalamic Circuits in Limbic Seizures
}

\author{
David Michael Sloan \\ Charlottesville, Virginia
}

A.S. Pre-Medicine, Brigham Young University-Idaho

B.S. Molecular Biology, Brigham Young University

A Dissertation presented to the Graduate Faculty of the University of Virginia in Candidacy for the Degree of Doctor of Philosophy

Edward H. Bertram, Dissertation Advisor

Neuroscience Graduate Program

University of Virginia

Aug 12, 2010

Kevin Lee

Peter Brunjes

Ignacio Provencio

Manoj Patel

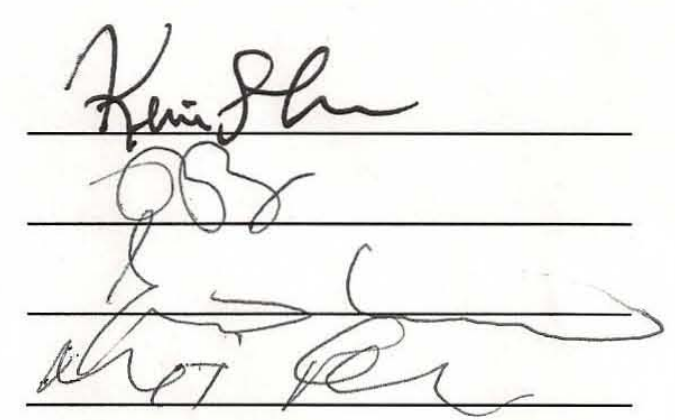




\section{Thesis Abstract}

The midline thalamus has been shown to have a critical role in limbic seizures. The presumed reason for its importance has been its widespread excitatory connectivity with the limbic network. However, the physiological relationship of the midline thalamus with respect to the network, and the ways in which that relationship contributes to seizure initiation and spread, are unclear. This thesis explores the relationship between the midline thalamus and the limbic network in animal models of limbic seizures. This includes: (1) an examination of signaling of the midline thalamus to the prefrontal cortex in rats with kindled or spontaneous limbic seizures, and how that signaling changes as the chronic model develops, (2) an examination of divergent-convergent circuits formed between the thalamus and two separate limbic pathways: the subiculum-prefrontal cortex and the entorhinal cortex-piriform cortex pathways, and how theses circuits may influence seizure spread, (3) an examination of how GABAergic modulation of thalamic neurons can have a widespread effect on limbic circuits, such as the subiculum-prefrontal cortex pathway. The conclusion of this thesis is that the role of the midline thalamus in limbic seizures can be derived, in part, from its extensive physiological influence on signaling within the limbic network. This work reinforces the evidence that the midline thalamus may make an effective clinical target for patients with mesial temporal lobe epilepsy. It also provides evidence that the role of the midline thalamus in the normal functional processes of the limbic network merit further exploration. 


\section{Table of Contents}

Chapter 1: Introduction

Part 1- Temporal Lobe Epilepsy: A Network Disorder..................................

Part 2- The Functional Anatomy of Limbic Seizures...................... 7

Part 3- The Midline Thalamus and Limbic Seizures........................10

Part 4- The Thesis.................................................. 17

Chapter 2: Changes in Midline Thalamic Recruiting Responses in the Prefrontal Cortex of the Rat During the Development of Chronic Limbic Seizures

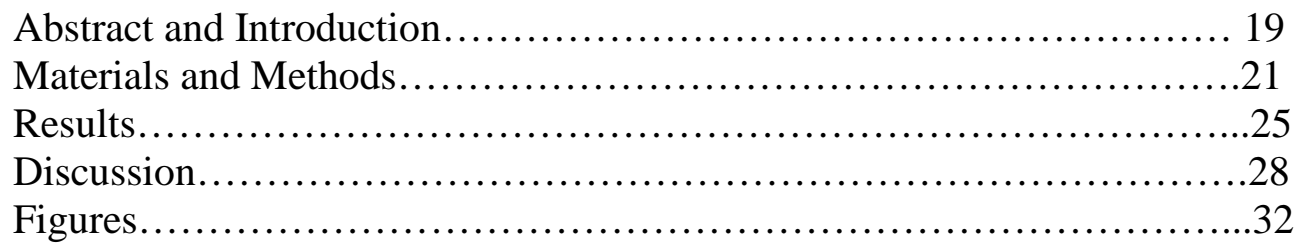

Chapter 3: Excitatory Amplification Through Divergent-Convergent Circuits: The Role of the Midline Thalamus in Limbic Seizures

Abstract and Introduction............................................37

Materials and Methods................................................

Results...........................................................

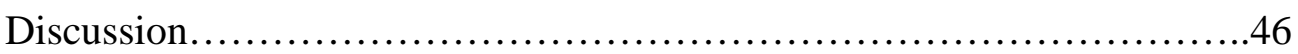

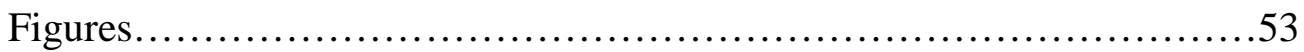

Chapter 4: Increased GABA-ergic Inhibition in the Midline Thalamus Affects Signaling and Seizure Spread in the Hippocampus-Prefrontal Cortex Pathway

Abstract and Introduction.................................................61

Materials and Methods...................................................63

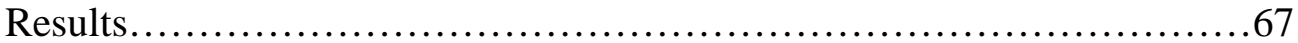

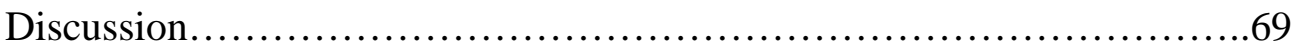

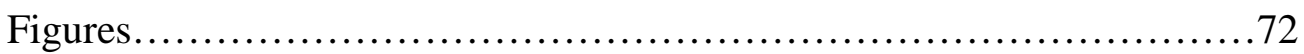

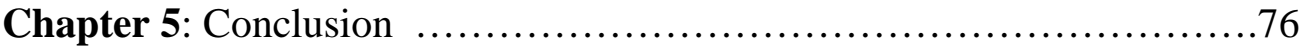

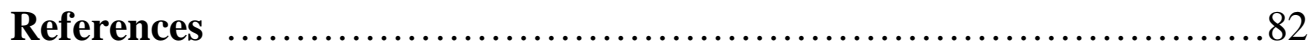




\title{
Introduction
}

\section{Part 1- Temporal Lobe Epilepsy: A Network Disorder}

\author{
Epilepsy
}

Epilepsy is a common neurological disorder that afflicts millions of people. A long history of clinical experience with epilepsy has generated an immense knowledge base of its symptoms, types, triggers and consequences for patients. As a result, many successful drugs and surgical treatments have been developed to combat the disorder. Nevertheless, an understanding of the neuronal mechanisms of epilepsy - its fundamental anatomical and physiological underpinnings - is still being researched, mostly because of the epilepsy patients for whom either drugs or surgery have not been sufficient to control their seizures. Any future improvements in clinical interventions for epilepsy patients depend on a more thorough grasp of the disorder's cellular, synaptic and circuit-based mechanics.

Seizures are the principal clinical symptom of epilepsy. A seizure is defined as a “clinical manifestation presumed to result from an abnormal and excessive discharge of a set of neurons in the brain... which may include alterations of consciousness, motor, sensory, autonomic, or psychic events, perceived by the patient or an observer.” Epilepsy is defined as the condition of having more than one spontaneous seizure, with no apparent provocation or reversible causes (Hopkins et al. 1995). According to the Epilepsy Foundation, epilepsy affects about 3 million Americans, with 200,000 new cases every year (epilepsyfoundation.org, 2010), making it one of the most significant neurology concerns in the country. The severity and treatability of an individual case depends greatly on the genetics, age, sex, and medical history of the patient, as well as the specific changes in the brain that allow seizures to occur. All these factors combine with the limited ability of the adult brain to heal to make epilepsy a very difficult disorder to suppress, much less cure. 
Epilepsy types are defined first by their apparent site of seizure onset. Focal, or partial, epilepsies have a broad region of onset that, based on EEG recordings, is consistent from seizure to seizure. Generalized, or absence, seizures have a less visible site of initiation, (although an initiation site may still exist) (Meeren et al. 2002) and seem to incorporate the whole cortex. Counterintuitively, focal epilepsies are more difficult to treat because generalized seizures are often caused by a single genetic or cellular anomaly (like a channelopathy) that can be targeted by systemic drug application. Focal seizures often include fundamental, permanent changes in multiple structures not just at the cellular level, but at the synaptic and circuit levels as well. Foremost in prevalence and notoriety among focal epilepsies is mesial temporal lobe epilepsy.

\section{Mesial Temporal Lobe Epilepsy}

Temporal lobe epilepsy (TLE) seizures often include aura, epigastric sensations, staring, restlessness and oroalimentary automatisms in early seizure stages, with more severe semeiology occurring later. In some cases, the seizure can spread far beyond the temporal lobe into other circuits (motor, sensory) in a process called secondary generalization. The particular manifestations of the seizures depend on the severity of the seizure, age of the patient, the proximity of the focus to subcortical limbic structures (like the hippocampus) and lateralization (Elger 2000). TLE, as would be expected with a physiological disruption of certain temporal lobe regions, is commonly associated with memory loss and emotional shifts, along with other forms of psychiatric comorbidity (van Elst et al. 2003). TLE is typically intractable and may require surgical removal of the focus to control seizure activity (Janszky et al. 2005). Currently, the success for temporal lobectomies performed to control mTLE seizures is between $60-80 \%$, leaving a significant portion of patients with seizures (Weibe et al. 2001, Bell et al. 2009).

The specific diagnosis of mTLE is given by the presence of visible sclerotic tissue in the hippocampus, first noted in post-mortem brain tissue in mTLE patients and now visualized with MRI. Since its discovery, evidence of cell death in a variety of other related structures has been found to be correlated with hippocampal sclerosis, giving the 
first indications that mTLE may be much more a disorder of a network rather than on a single region.

\section{Animal Models of mTLE}

To understand better how and why mTLE seizures occur, various animal models have been created. As these models have evolved in sophistication, the precision and malleability these models have increased, leading to findings that are more translational for clinical practice. The results of work with these models in clarifying the functional circuitry of limbic seizures will be discussed throughout this thesis.

The earliest animal models were created with systemic administration of a convulsant such as penicillin, bicuculline, flurothyl, pilocarpine and kainic acid. Administration of these drugs interparietally, or directly onto the cortex, cause generalized seizures. This yielded some important discoveries about the nature of convulsants and anticonvulsants, as well as some of the basic circuitry of generalized seizures, like the importance of thalamocortical loops in generalized seizure synchrony (Avoli and Gloor, 1982). Still, the imprecise nature of these seizures had experimental drawbacks.

In 1969, Goddard et al. furthered seizure research significantly by introducing the electric kindling model. They showed that an electrode could be placed in a normal, nonconvuslive animal, in a given brain area, and regular electrical stimulations at high frequency could be given. In this way, animals would develop increasingly severe seizures with each stimulation. This technique allowed for seizures to be introduced with greater consistency and exactness, and allowed for drug injections or interventions to be timed precisely with seizure onset. Kindled seizures can also be induced while the animal is under anesthesia, although in this case there is no behavioral seizure manifestation, and the anesthesia may make it more difficult for the seizure to be generated.

The great strength of the kindling model can also be a weakness. The lack of spontaneity of the seizure activity is useful in a laboratory setting, but there is little clinical correlation to the spontaneous seizures that occur in most epilepsy patients. 
Kindling models also don't have the cell death seen in mTLE, including hippocampal sclerosis, although there are clearly physiological changes that take place (Bertram et al. 2001, Racine et al. 2002).

A modified protocol yields a more applicable model. A prolonged electrical stimulation, or a focused dose of convulsant in a cannula, can be applied directly to a brain region, inducing a long bout of status epilepticus (prolonged, uncontrolled seizing). The uncontrolled seizure causes damage to a particular set of networked regions. After a period of weeks or months, this damage will induce a rewiring of the local circuitry in each of those regions (Ben-Ari et al. 1981, Cavalheiro et al. 1982, Brandt et al. 2003, Dinocourt et al. 2003). The animals will begin to experience spontaneous seizures chronically (Lothman et al 1990). When these seizures are induced in a region like the hippocampus, it is the model that most closely mirrors human mTLE. The important factor for the development of a useful mTLE model is one that replicates the pattern of cell death seen in human patients (at least in chronic seizure models).

The pattern of cell death in mTLE aligns with a set of cortical and subcortical connections known as the limbic system, or limbic network. Therefore, models of mTLE are referred to as having chronic limbic epilepsy. The terms mTLE and limbic epilepsy will be used interchangeably hereafter.

\section{Part 2: The Functional Anatomy of Limbic Seizures}

Because limbic network connectivity seems to be at the heart of mTLE, an understanding of what the network is and how it operates is critical for understanding the changes it undergoes in the development of epilepsy.

\section{The Limbic Network}

The idea of a limbic network or system has undergone many revisions since the early days of Broca and Papez. Originally defined anatomically and then clinically, its borders have grown, its functions are better understood and its significance is constantly reexamined. The overall function of the limbic network is to subserve emotional 
processing, learning, memory and contextual awareness. The hippocampus and amygdala are the most well-known and intensely studied representatives of the network, although each structure has an important, related function. Obviously, any disorders or lesions affecting limbic network function can result in serious behavioral abnormalities. Disfunctions of the network can lead to depression, apathy, amnesia, mania, obsession/compulsion, anxiety, memory loss and psychosis, among others (Mega et al. 1997). The tight connectivity and plasticity of the network also makes it particularly vulnerable to seizures.

In primates, much of the essential limbic network regions are located in the temporal lobes and medial midbrain (Isaacson 2002, Mega et al. 1997). In rats, all the same regions exist, albeit with differences in geography. For the purposes of this thesis, the limbic network incorporates a set of functionally interconnected structures* that include the amygdala, hippocampus and parahippocampal region, entorhinal and piriform cortices and the midline/anterior thalamus. By its definition, the limbic network could be expanded to include parts of the basal ganglia, forebrain, brainstem and frontal cortex, but expanding the definition too much can quickly become unwieldy. The regions mentioned are of note because of their observed involvement in limbic seizures.

\section{Circuitry of Limbic Seizures}

In 1966, Margerison and Corsellis, correlating EEG, case history and postmortem studies, found that the most common brain regions damaged in TLE patients were, in order: the hippocampus, cerebellum, amygdala, and thalamus (Margerison and Corsellis 1966). This gave an indication that, while temporal lobe structures were certainly affected by seizures physically, this effect was not limited to the temporal lobe; several parts of the brain could become involved.

In the early 1980's a number of studies using radioactive 2-deoxyglucose absorption were preformed to examine what structures were activated during seizures that were kindled in the amygdala (Engel et al. 1978, Blackwood et al. 1981, Lothman et al. 1985). The results of these studies were slightly mixed, but there were structures that consistently stood out: the hippocampus and amygdala (even when they were not the 
kindled target), substantia nigra pars reticulata, portions of the basal ganglia, and the thalamus. The study by Lothman et al. included physiological recordings of seizures throughout many of those regions, confirming their involvement. This work began to confirm that there was, as the Lothman group called it, a "functional anatomy" of limbic seizures that needed to be better understood.

Research into the specific changes induced by limbic seizures that occurred in these relevant structures led to a huge outpouring of data. The summation of these data is that the onset of seizures was accompanied by dramatic anatomical and physiological changes in each structure. The nature of the changes was different according to the type and severity of the seizures induced, but the finding was that, in each affected structure, the changes provided an environment of net hyperexcitability. The logical progression of that finding was that the relationship between these structures was altered as well, and that the net environment of the network of limbic structures was hyperexcitable, thus allowing for seizures to initiate and spread.

\section{Hippocampus-EC and Seizure Circuitry}

The most studied alterations in regional circuitry due to limbic seizures are those found in the circuit that links the hippocampus with the entorhinal cortex. Chronic seizure models cause massive changes at almost every step of this pathway. Signals pass through this pathway in this general order: dentate gyrus $(\mathrm{DG}) \rightarrow \mathrm{CA} 3 \rightarrow \mathrm{CA} 1 \rightarrow$ subiculum (SB) $\rightarrow$ deep layers of the entorhinal cortex (EC) $\rightarrow$ superficial EC $\rightarrow$ DG. That is an oversimplification of the relationship of each subregion, but it is the general order based on anatomical proximity.

In chronic seizure models, the changes that result from the initial insult and the subsequent recovery are staggering. Neurons die throughout the circuit, including interneurons, and layers shrink in volume. Neurons in the DG sprout dendrites and form a mesh of local microcircuits to compensate for the cell loss. CA3 cells sprout as well, sending projections as far as the subiculum and EC. Changes in synaptic responsiveness, receptor distribution and other factors combine with the gross physical changes to 
encourage inappropriate neuronal excitability in the entire pathway (Mangan 1997, Saddiqui 2005, Knopp 2005, Cavazos 2006, Sun et al. 2007).

For this thesis, the notable thing about the alterations in the hippocampal-EC pathway in epilepsy is the distribution of potentially epileptiogenic zones. A seizure could theoretically start at any point in the circuit and spread then quickly spread throughout the circuit. The fundamental change in the connectivity of the hippocampus and EC encapsulates a functional anatomy of seizure spread. In the same chronic seizure models, the same could be said of the connectivity between the EC and amygdala, the amygdala and the piriform cortex, and so on throughout the limbic network. In fact, the idea of a "focus" in such a network is very fluid, and may not truly exist. Models of epilepsy have demonstrated that limbic seizures rarely initiate in the same place or spread through the same pathways with every occurrence (So 1991, Spencer and Spencer 1994, Bertram 1997).

When considering this functional anatomy, it should become clear that some structures may be more important than others in initiating and spreading seizures in the network. Such importance would be determined by the range and influence of its afferents. Identifying such structures is important because it they would represent more powerful targets for clinical interventions on limbic seizures. Several structures seem to be important in this way, but one in particular stands out, and it is the main subject of this thesis.

\section{Part 3: The Midline Thalamus and Limbic Seizures}

As mentioned throughout this introduction, one structure that is often correlated with symptoms of mTLE and limbic seizures is the thalamus. Cell death in the thalamus was first correlated with hippocampal sclerosis in the 1960's (Margerison and Corsellis 1966). This correlated cell loss, connected with a loss of glucose metabolism, has been confirmed in multiple studies (Juhasz et al. 1999, Gong et al. 2008, Mueller et al. 2009, Kim et al. 2010), and has been confirmed in animal models of chronic seizures (Bertram and Scott 2000, Bertram et al. 2001). It was activated in the 2-deoxyglucose studies of 
kindled limbic seizures (Lothman et al. 1985, Cassidy and Gale 1998). From this evidence, the thalamus would seem to be a clear part of the functional anatomy of limbic seizures. Closer examination of the connectivity of this structure reveals more intriguing possibilities for a potentially powerful role in limbic seizures (Figure 1).

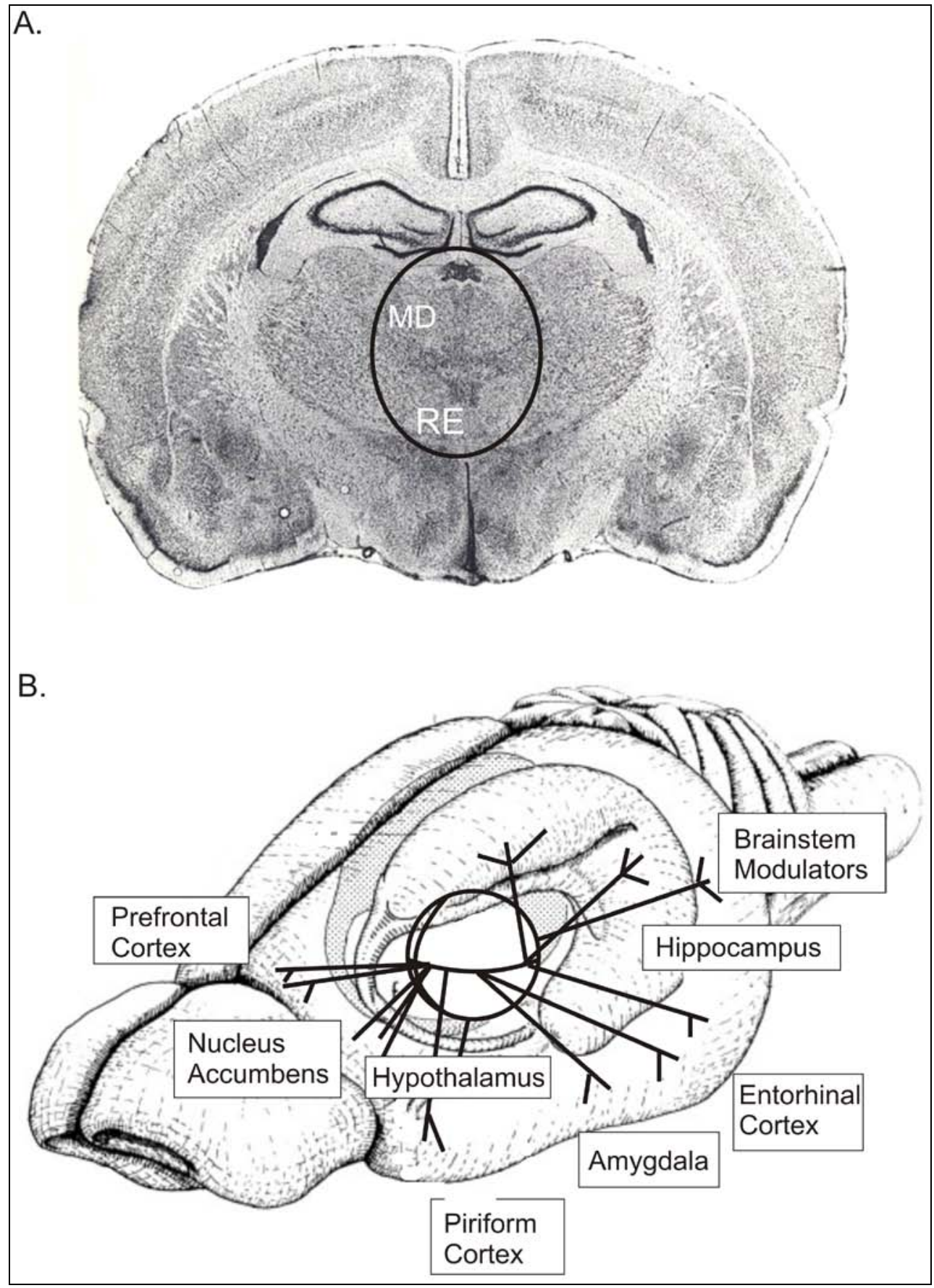

Introduction Figure 1: The Midline Thalamus 
(A) Coronal section of the Rat Brain (Paxionos and Watson 1996) with the region of the midline thalamus circled. The Mediodorsal and Reuniens nuclei are noted. (B) Representation of the rat brain with the midline thalamus at center. Dark lines represent connections (efferent, afferent or reciprocal) to multiple regions of the brain. Note that the entire frontal and temporal regions are connected in to the midline thalamus. (Brain outline from www.biomedcentral.com, 2010).

\section{Anatomy of the Midline Thalamus}

The portion of the thalamus that is most closely associated with the limbic network is the midline thalamus. In the rat, the midline thalamus is defined as the most medial region of the thalamus, consisting of several nuclei that sit between the ventrolateral thalamic areas, beneath the dorsal hippocampus, and above the third ventricle. It consists of multiple nuclei, which can be broadly subgrouped into anterior, mediodorsal and ventral divisions. The mediodorsal nuclei include the central median, paraventricular and the mediodorsal nucleus itself. The ventral include the rhomboid and reuniens. The purpose of dividing them up into subgroups is more practical than functional, as many subgroups overlap in both afferent and efferent connections with each other (although each nucleus has a distinct projection milieu, and there is a clear gradient of thalamic connectivity in many cortical regions (Ray et. al. 1992, Van der Werf et al. 2002, Berendse and Greoenewegen 1991, Hoover and Vertes 2007). The midline thalamus in primates is larger, more developed and contains more nuclei (Morel et al. 1997, Behrens 2003).

Collectively, the midline thalamic nuclei connect to every region of the limbic network (Van der Werf et al. 2002, Berendse and Greoenewegen 1991, Hoover and Vertes 2007). Targets also include the prefrontal cortex, basal ganglia and ventral forebrain. Of note is that there is no structure implicated in the functional anatomy of limbic seizures that does not have a connection with the midline thalamus. Although thalamic afferents are glutamatergic, they often innervate both excitatory inhibitory cells, as shown in both cortex (Cruikshank et al. 2010) and hippocampus (Dolleman-Van der Weel et al. 1997). Furthermore, inputs to the thalamus can be subdivided functionally by the cortical layer they come from (Reichova and Sherman 2004).

Multiple modulatory influences feed into the midline thalamus. GABAergic interneurons feed in from the reticular nucleus, which encapsulates the thalamus and receives feedback signals from many thalamic cortical targets (Nagaeva 2006). Some 
structures of the ventral forebrain, such as the ventral pallidum and globus pallidus also send GABAergic projections in (Churchill 1996), as does the substantia nigra pars reticulata (Tanibuchi 2009). The midline thalamus also receives serotonergic (Vertes 2010), dopaminergic (Garcia-Cabezas et al. 2009) and adrenergic (Vogt et al. 2008) inputs as well, indicating that midline thalamic output is regulated in a complicated manner.

Functionally, studies have linked the midline thalamus to processes associated with the limbic network. It has been particularly implicated in learning and memory functions (Powell and Churchwell 2002, Van der Werf et al. 2003, Mitchell et al. 2007, Lopez et al. 2009).

In summary, midline thalamic connectivity is extensive, ubiquitous, and functionally relevant to limbic network processes. Anatomically, the midline thalamis is ideally placed to participate in limbic seizures to a high degree, and it could perform any number of roles. Once the implications of the anatomy were understood, the next step was to test the criticality and activity of the midline thalamus in limbic seizures.

\section{The Epileptic Midline Thalamus}

Early studies of generalized seizures were the first to identify a role for thalamocortical circuitry in seizure activity. For example, manipulation of thalamocortical links in cat models demonstrated that, although the thalamus wasn't the initiating site of the seizures, its circuitry was critical for the synchronization of seizure activity across the cortex (Pellegrini et al. 1979, Avoli and Gloor 1982). Subsequent research showed that thalamic circuitry was a key driver of cortical seizure activity in genetic absence models (Meeren et al. 2002) and in the organization of synchronous cortical EEG phenomenon like sleep spindles (Steriade 1987, Meeren 2009).

In 1988, researchers at the Meldrum lab in London published a study in which they suppressed acute pilocarpine seizures by injecting the selective NMDA antagonist APV or the GABA agonist muscimol into the mediodorsal nucleus. They found that, in both cases, seizures were reduced or suppressed in a dose-dependent manner (Patel et al. 1988). Miller and Ferrendelli confirmed that work independently by injecting a variety 
of GABA agonists into the central median nucleus (which sits between the mediodorsal nuclei), and found a dose-responsive modulation of behavioral seizure activity caused by systemic bicuculline. In their conclusions, they postulated that the central media nucleus was not "a site of seizure origination or spread but rather acts to modify the excitability of other structures more directly involved in this process” (Miller and Ferendelli 1990).

Nearly a decade later, the Gale lab at Georgetown published a similar study in which they induced limbic seizures by injecting bicuculline into a specific subregion of the piriform cortex, in order to study the role of the thalamus within the context of a more controlled limbic seizure circuitry. Autoradiography of slices using radioactive 2deoxyglucose injected 15 minutes prior to seizure induction showed a strong involvement of the midline thalamus, in conjunction with temporal cortical involvement. They went on to show that injection of the AMPA agonist NBQX and muscimol into the midline thalamus just prior to seizure induction suppressed behavioral seizures (Figure 2- Cassidy and Gale 1998). Around the same time, a study of neural death in the pilocarpine model showed bilateral damage in the midline thalamus, implying that there was a pathological result of seizures in the thalamus instead of merely a physiological involvement (Turski et al 1986).

Around the same time as the Gale study, Bertram and colleagues began to examine the role of the thalamus in electrically-induced chronic seizures. In 1998, they published a study that showed electrographic thalamic involvement of the midline thalamus in status epilepticus that was synchronous with other limbic structures. They also showed that responses to thalamic stimulation throughout the network were more excitable in rats with post-status spontaneous seizures, leading them to suggest that the thalamus “is well suited to play the role of seizure activity synchronizer” (Bertram et al. 1998). Later, they confirmed electrographic involvement of the midline thalamus in both chronic kindled and spontaneously-seizing animals. Thalamic cells in slices from epileptic rats showed bursting behavior, and lidocaine was added to the list of drugs that could be injected into the thalamus to block seizure activity (Figure 2- Bertram et al. 2001). Further studies during this time sought to characterize the normal interactions 
between the thalamus and limbic structures in order to better understand their role in seizures (Bertram and Zhang 1999, Zhang and Bertram 2002). This work helped to form the foundation for the studies described in this thesis.

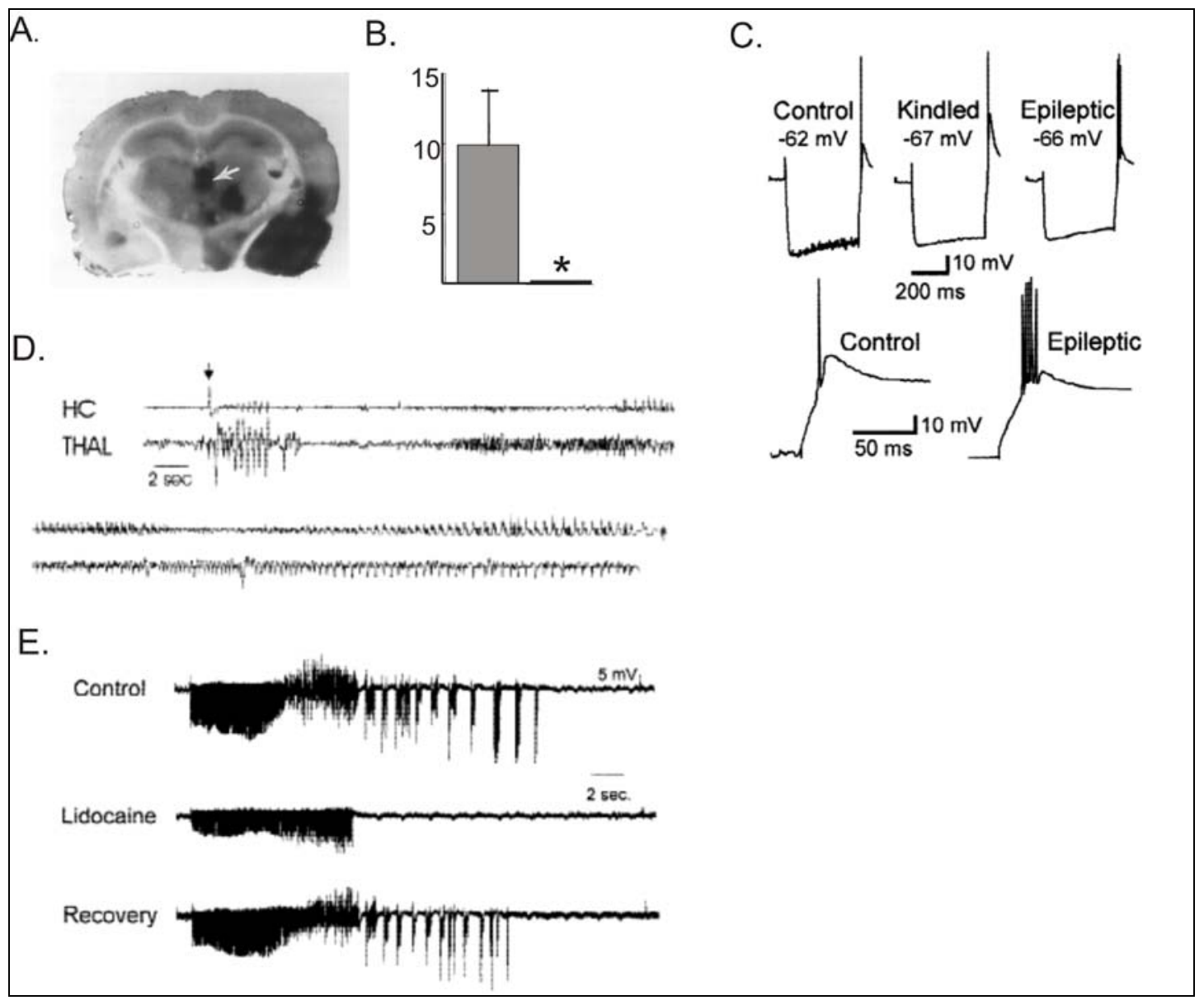

\section{Introduction Figure 2: Evidence for the Role of the Midline Thalamus in Limbic Seizures}

Representative figures from papers examining the role of the midline thalamus in limbic seizures. (A) and (B) from Cassidy and Gale 1998. (A) Radioactive 2-Deoxyglucose marks areas of increased excitation in a piriform kindled rat. Note that, in addition to temporal cortex, the midline thalamus is marked. (B) Injection of glutamate antagonist NBQX injected into the midline thalamus stops behavioral seizures in kindled rats. (C-E) from Bertram et al. 2001. (C) Voltage clamp experiment showing results of hyperpolarization on thalamic cells in normal, epileptic and kindled animals. Cells from epileptic animals are prone to bursting behavior. (D) Spontaneous electrographic seizure recorded in the midline thalamus occurring simultaneously with hippocampus. (E) Injection of sodium channel blocker lidocaine in the midline thalamus stops kindled seizure activity. 


\section{Clinical Future of the Midline Thalamus}

The evidence to date regarding the importance of the midline thalamus to mTLE has not escaped attention in the medical community. Some preliminary work has been done to use deep brain stimulation techniques on the thalamus, specifically the anterior nuclei, as a treatment for temporal lobe epilepsy. The majority of these studies have been attempted in the last decade, although some initial forays were made in the 1980's.

Most recently, a national study group, SANTE (Stimulation of Anterior Nucleus of the Thalamus in Epilepsy), reported that regular bilateral stimulation of the thalamus in patients with partial seizures significantly decreased seizure frequency over a two year period. The relative benefit of the stimulation was higher for those with temporal lobe seizures. Interestingly, the authors of that study wrote in their conclusions that "[the reasons] why electrical stimulation of the thalamus reduces seizures remote from the stimulation site is presently unknown.” (Fisher et al. 2010).

The study was conducted targeting the anterior nuclei of the thalamus, which, although close to the midline nuclei, has a slightly different input/output milieu. It's possible that the cases in the study where stimulation did not work could be attributed, in part, to being too far from that more critical set of nuclei.

\section{The Case for the Midline Thalamus}

To summarize from the previous sections, the following is known about limbic seizures and the midline thalamus.

(1) Limbic seizures involve much of the limbic network.

(2) The midline thalamus connects to the entire limbic network

(3) The midline thalamus participates in limbic seizures. 
(4) The midline thalamus is damaged and altered in limbic seizures- both in humans and animal models.

(5) Midline thalamic efferents are excitatory.

(6) Thalamocortical processes are critical in other forms of epilepsy.

(7) Lesion or modulation of the midline thalamus affects limbic seizures.

(8) Medical interventions targeting the thalamus are reporting preliminary success.

With these facts to build on, it can be hypothesized that the thalamus plays any number of roles in limbic epilepsy.

(1) Initiator: As a structure that sends excitatory signals to limbic circuits that, in the course of developing epilepsy, have become hyperexcitable, it's possible that the thalamus assists in sparking seizure activity.

(2) Distributor: The expansive connectivity of the thalamus make it ideal as a router of seizure signaling throughout the network.

(3) Facilitator: Its physiological relationship with limbic structures may be key to recruiting and synchronizing limbic circuitry into the seizure.

Clearly, the midline thalamus is a major subject of interest in limbic seizure research. However, many questions remain regarding the details of its role. This thesis attempts to answer some of those questions.

\section{Part 4: The Thesis}

The purpose of the studies in this thesis is to provide a better understanding of the role of the midline thalamus in the initiation and spread of seizures. The overriding hypothesis is that the important characteristic of the midline thalamus in seizures is its 
widespread influence on signaling within the limbic network. In epilepsy, the normal relationship between the thalamus and limbic circuits is altered, such that its normal functional connectivity is used to facilitate seizure activity throughout the network.

To the end of investigating that idea, we had three specific goals:

(1) Identify how signaling between the midline thalamus and a cortical target is altered in limbic seizure models.

(2) Identify the role of midline thalamic signaling on distinct limbic circuits with regards to seizure activity.

(3) Identify the effect of modulation of the thalamus on distinct limbic circuits.

These questions are addressed in the three main sections of the thesis.

In Chapter II of this thesis, entitled “Changes in Midline Thalamic Recruiting Response in the Prefrontal Cortex of the Rat During the Development of Chronic Limbic Seizures" the interaction between the midline thalamus and the medial prefrontal cortex is explored. The object of this study is to demonstrate that direct signaling between the midline thalamus and its afferent target is affected in a model of chronic limbic seizures. We use a unique physiological marker of thalomocortical signaling, the recruiting response, to explore how this signaling changes as the chronic seizure model develops.

In Chapter III, entitled "Excitatory Amplification Through Divergent-Convergent Circuits: The Role of the Midline Thalamus in Limbic Seizures,” the indirect influence of the thalamus on signaling in two different limbic circuits is explored.

In Chapter IV, entitled "Effect of GABAergic Modulation of the Midline Thalamus on Subicular Signaling and Seizure Spread to the Medial Prefrontal Cortex”, the work in section II is expanded to examine the effect of GABAergic modulation of the midline thalamus on one of those circuits.

*Note, pg 8: There is a question about the definition of the definition of the prefrontal cortex in the rat, compared to humans, and what qualifies as "prefrontal” and "cingulate” cortex. This is a question of anatomical definition that is beyond the scope of this work. For this thesis, the rat prefrontal cortex 
consists of the Anterior Cingulate and Prelimbic cortices, and are considered limbic system-associated targets.

\section{Chapter II}

\section{Changes in Midline Thalamic Recruiting Responses in the Prefrontal Cortex of the Rat During the Development of Chronic Limbic Seizures}

Summary

Purpose: Mesial Temporal Lobe Epilepsy (MTLE) is a common form of epilepsy that affects the limbic system and is associated with decreases in memory and cognitive performance. The medial prefrontal cortex (PFC) in rats, which has a role in memory, is associated with and linked anatomically to the limbic system, but it is unknown if and how MTLE affects the PC.

Methods: We evoked responses in vivo in the PC by electrical stimulation of the mediodorsal (MD) and reuniens (RE) nuclei of the thalamus at several time points following status epilepticus, before and after onset of spontaneous seizures. Kindled animals were used as additional controls for the effect of seizures that were independent of epilepsy.

Results: Epileptic animals had decreased response amplitudes and significantly reduced recruiting compared to controls, whereas kindled animals showed an increase in both measures. These changes were not associated with neuronal loss in the PC, although there was significant loss in both the MD and RE in the epileptic animals. 
Conclusions: There is a significant reduction in the thalamically-induced evoked responses in the PCs of epileptic animals. This finding suggests that physiological dysfunction in MTLE extends beyond primary limbic circuits into areas without overt neuronal injury.

\section{Introduction}

Mesial temporal lobe epilepsy (mTLE) is a neurological disorder involving chronic, spontaneous seizures originating in the limbic structures of the temporal lobe (Engel 1996). MTLE in humans and animal models is associated with cell damage and circuitry change (Margerison and Corsellis 1967, Yilmazer-Hanke et al. 2000, Morimoto et al. 2004). Damage to limbic circuits is thought to be the basis for observed memory disruption in mTLE patients, as the limbic system is crucial for learning and memory processes (Glowinski 1973, Viskontas et al. 2000, Voltzenlogel et al. 2006).

The prefrontal cortex (PFC) is associated with memory functions, as well as higher-level functions such as executive processing and decision-making (Deacon et al. 2003, Price 2005, Macrae et al. 2004). The PFC is reciprocally connected to limbic structures known to be involved in and affected by mTLE (Vertes 2004, Hoover and Vertes 2007, Margerison and Corsellis 1967). Despite these links, the degree to which the PC is affected in MTLE is unknown. PET studies have shown signs of glucose hypometabolism in the PFCs of mTLE patients (Jokeit et al. 1997, Takaya et al. 2006). However, the basis for these changes is unclear.

In this study, we sought to identify potential changes in the input to the medial PC from the midline thalamus in a rat model of mTLE, focusing on two distinct nuclei with overlapping prefrontal projections: the Mediodorsal (MD) and Reuniens (RE). These thalamic nuclei were chosen because they are closely connected to the limbic system (Van der Werf et al. 2002, Giguere et al. 1988, Berendse et al. 1991, Gabbott et al. 2005), 
and are thought to participate in the initiation and spread of limbic seizures (Bertram et al. 2001, 2008, Cassidy and Gale 1998).

To study the thalamic connection to the PFC in epilepsy, we used trains of stimuli to induce the thalamic recruiting response, which is a progressive augmentation of the amplitude of the response wave which plateaus by the $4^{\text {th }}$ stimulation in the train (Figure 1). This response was first described in the early 1940's (Dempsey and Morison 1942, Morison and Dempsey 1943), and is strongly linked to midline thalamic stimulation and cortical excitation (Verzuano et al. 1953, Herkenham 1986, Bazhenov et al. 1998). Since the PFC shares connections with a variety of structures that are also networked to the midline thalamus, it was important to examine a cortical response that was strongly linked to thalamic input. The recruiting response is also a useful tool for examining the PFC-midline thalamic connection because the maximal amplitude of the response in the recruiting rhythm is much larger than the response to single stimuli.

\section{Materials and Methods}

All animals were used under a protocol approved by the Animal Care and Use Committee (ACUC) of the University of Virginia.

\section{Groups for Study}

We grouped our animals using predetermined time points, based on a known progression of alterations in physiology (Mangan and Bertram 1998). We used six groups in this study:

(1.) Naïve, unstimulated controls ( $\mathrm{n}=12)$.

(2.) Groups experiencing status epilepticus (SE), taken at time points prior to evolving spontaneous seizures:

(a) 2 days ( $\mathrm{n}=5)$, for an acute measure following SE.

(b) 7 days ( $n=6)$, at which point cell death is expected to be maximally apparent (Dinocourt et al. 2003). 
(c) 14 days $(\mathrm{n}=6)$ for a measure during the late latent period, before onset of spontaneous seizures (Bertram and Cornett 1994, Mangan and Bertram 1998).

(3.) Epileptic ( $n=6)$, with multiple spontaneous seizures at least 2 months after SE.

(4.) Kindled ( $n=6)$, to determine whether observed changes in the epileptic animals were secondary effects related to seizure activity in the absence of the pathology seen in epileptic animals (Bertram et al. 2001, Mangan et al. 2000, Racine et al. 2002).

Surgery

The method for creating chronic seizure models by electrical stimulation of the hippocampus, for both epileptic and kindling models, has been described previously (Lothman et al. 1990). Briefly, adult Sprague-Dawley rats (250-300 grams) were implanted with a single, twisted pair, stainless steel bipolar electrode while under ketamine/xylazine anesthesia (50 mg/kg ket.,10 mg/kg xyl.). During all surgeries, the bite bar was set at $-3.3 \mathrm{~mm}$. The electrode was placed in CA1 of the mid-ventral hippocampus,(from Bregma, -5.6 mm AP, +4.9 ML, -5 mm DV) and secured with dental acrylic and skull screws (Paxinos and Watson 1986).

\section{Status Epilepticus Animals}

Five days after the surgery, the animals were stimulated with a train (50 Hz peak to peak, $400 \mu \mathrm{A}, 1 \mathrm{~ms}$ biphasic square-wave for 10 seconds, delivered every 11 seconds), after which the rats went into a period of status epilepticus for 10-14 hours. They were then allowed to recover. Headsets were removed under anesthesia prior to the physiology experiments. In epileptic rats, spontaneous seizures were confirmed using 24-hour video surveillance simultaneously with EEG recording. Rats having a minimum of 2 spontaneous seizures were considered epileptic. Data were recorded using Stellate HARMONIE-E version 6 software. Identification of seizure activity has been described (Bertram 1997). 


\section{Kindled Animals}

Kindled rats received stimulations ( $50 \mathrm{~Hz}$ peak to peak, $400 \mu \mathrm{A}, 1 \mathrm{~ms}$ biphasic square-wave, lasting 10 seconds) every hour, 6 times daily, every other day. Animals were considered fully kindled when they demonstrated rearing and falling behaviors (Racine Class V seizures). This protocol has been outlined in detail previously (Lothman and Williamson 1993). Kindling until the first motor seizure required an average of $15 \pm$ 2 stimulations, after which they received an average of $20 \pm 3$ additional stimulations to ensure that all animals were equally and adequately kindled. Animals were used no sooner than 48 hours after their most recent kindling stimulation.

\section{Electrophysiology Experiments}

Rats were put under urethane anesthesia (1.2g/kg, i.p.) and placed in a stereotactic frame in a Faraday cage. A twisted-pair bipolar steel electrode with a diameter of .56 $\mathrm{mm}$, with the tips separated by $.5 \mathrm{~mm}$ at an angle, was placed in the midline thalamus on a trajectory aimed to pass through the MD and RE thalamic nuclei (from Bregma: $-2 \mathrm{~mm}$ AP, 0.5 mm ML, 4.5-7.5 mm DV, $5^{\circ}$ angle). A glass recording pipette of approximately $1 \mathrm{M} \Omega$ resistance was filled with $0.9 \%$ saline solution and Fast-Green dye and connected to the amplifier through a silver-silver chloride wire. It was placed in the medial PFC (from Bregma: $3.5 \mathrm{~mm}$ AP, $0.4 \mathrm{~mm} \mathrm{ML}, 1.5-4 \mathrm{~mm} \mathrm{DV}, 2^{\circ}$ angle from vertical). This trajectory includes the Anterior Cingulate areas (Paxinos and Watson 1986).

Stimulations were delivered using a Winston pulse train timer, which drove a separate constant-voltage SIU. Stimulation was started at $15 \mathrm{~V}$ and then increased by 10 $\mathrm{V}$ until the response amplitudes plateaued, as defined by increased stimulation intensity without increased response amplitude (measured resistance across stimulating electrodes $30 \mathrm{k} \Omega$, maximum calculated maximum current $2.3 \mathrm{~mA}$ ). Only maximal responses were used for analysis. Recordings were obtained using an Axon Smartprobe, which was connected to a Cyberamp 380 amplifier and digitized with an Axon Digidata 1200. Recordings were viewed and measured using AxoScope 8.2 software. Filtering during recordings was set to $.5 \mathrm{kHz}$ lowpass and $2 \mathrm{~Hz}$ highpass, with a digitizer gain of 2 . 
The recruiting rhythm stimulation protocol consisted of trains of stimuli of five $0.2 \mathrm{~ms}$ pulses with an inter-stimulus interval of $120 \mathrm{~ms}$. Responses to ten trains (given at 10 second intervals) were averaged for each stimulation position. The response amplitudes and latencies were measured for the $1^{\text {st }}$ and $4^{\text {th }}$ stimulation responses, as measured from the baseline at stimulus onset. Responses were measured from the baseline value occurring immediately before the stimulation to the highest-amplitude positive peak following stimulation (Figure 1C).

We used two stimulation sites: the MD (5-5.5 mm below surface) and the RE (6.5-7 mm below surface). For each stimulation site, we dropped the recording electrode in increments of $.5 \mathrm{~mm}$ in a range of 1-4 $\mathrm{mm}$ below the cortical surface into the medial PFC. Responses of maximal amplitude, as measured at the $4^{\text {th }}$ response wave in the train, were obtained for each stimulation site for each animal and used for our analysis, and the depths at which those responses were obtained were noted.

Following the experiment, the rats were perfused with $4 \%$ paraformaldehyde. Their brains were removed and placed in fixitive with 20\% glucose for at least 48 hours. They were then frozen, sectioned, and examined to confirm the position of the electrode tracts. In cases where one or more electrodes were found to be outside the regions of interest, the data from those animals were not included in the analysis.

\section{Seizure Recording}

Because the presence or absence of seizure activity in the recording site could influence the responses and cause differences between groups, we determined if there was a difference in seizure involvement in the PFCs of kindled $(n=4)$ and epileptic ( $n=2)$ animals. To record seizures, twisted, bipolar stainless steel electrodes were placed in the standard position in CA1 of the hippocampus, as well as the medial PFC (from Bregma: +3.5 mm AP, $+0.5 \mathrm{~mm} \mathrm{ML,} \mathrm{2-4.0} \mathrm{mm} \mathrm{DV,} 2^{\circ}$ angle). Animals were allowed to recover for 5 days before recording. Recordings were made from the start of the kindling process in order to observe the evolution and progression of seizures in the PFC with repeated stimulations. A minimum of two seizures were obtained for each epileptic animal, while a minimum of 20 were obtained for each kindled animal. The principal purpose of this 
data collection was to determine if PFC was involved in seizure activity, and therefore we did not further quantify aspects of the seizures that we recorded.

The method for EEG data acquisition has been described earlier (Bertram et al. 1997). EEG recording was accompanied by simultaneous video recording. Epileptic rats were monitored continuously $24 \mathrm{hrs} /$ day. Data from kindled animals were recorded during the times in which they were stimulated, with recordings ending 10 minutes after the stimulation. After recordings were obtained, brains were fixed and sectioned to confirm the correct placement of the electrodes.

\section{Silver Staining}

To determine if cell death in the PFC following SE may have a role in the changes found in our experiments, we performed a silver-staining procedure on a group of 7 Day post-SE rats ( $\mathrm{n}=5)$ and controls $(\mathrm{n}=4)$. Silver stain is believed to stain dying or injured neurons preferentially. Animals used for silver staining were not involved in electrophysiological experiments. 7 days following SE, their brains were perfused, stored, and sectioned as described. All silver staining was done using the Neurosilver Kit by FD Neurotechnologies (Catonsville, MD, USA), following their protocol. Sections were kept from light during and after the process. Stained slides were dehydrated and coverslipped for examination.

Slides were examined qualitatively with a standard light microscope. At low magnification, regions of positive-staining neurons were identified as grayish-black areas against a golden-brown background. At higher magnification, individual neurons were identified as being positively stained if they were blackish in color, as opposed to brown. Previous studies have found no evidence for neuronal damage in our kindled animals (Bertram et al. 2001, Racine et al. 2002); therefore we did not stain kindled brains for comparison.

\section{Results}

Response Characteristics 
Recruiting responses were elicited in the PFC by stimulation of both the MD and RE nuclei. The qualitative nature of the response waveforms varied with the recording depth but there was a consistent anatomic region in which the responses of maximal amplitudes were obtained (Table 1), which corresponded to layers III-V of the anterior cingulate gyrus, also known as cingulate areas 1 and 3 (Paxinos and Watson 1989). These maximal electrode placements remained consistent among the groups that were subsequently studied.

Typical responses included a positive wave that increased with each subsequent stimulation until the $4^{\text {th }}$ stimulation, at which point response waves plateaued at an amplitude that was, on average, 3-4 times greater than the amplitude after the $1^{\text {st }}$ stimulation. The latency to the onset of this wave, as well as the latency to its peak, was consistent (Table 1). The recruiting wave was usually followed by a prolonged, loweramplitude negative wave (see Figure 1). Outside of our selected regional boundaries, waves of the same latency as the recruiting wave analyzed were often negative and nonrecruiting (Figure 1A). Maximal amplitudes were obtained, normally at a stimulation intensity between 50-60 V. (Figure 1B).

\section{Comparison of Responses}

We compared the recruiting responses of control animals to those of epileptic animals (Figure 2A). Responses qualitatively similar to those seen in control animals were elicited at similar electrode depths, and the characteristic response latencies were also unchanged (Table 1 and Figure 1). However, $1^{\text {st }}$ response waves from the epileptic animals were smaller than those in control responses, with the difference significant following RE stimulations (Table 2). Fourth response waves were also significantly smaller at both stimulation sites. Recruiting responses were significantly attenuated but not eliminated in epileptic animals; $4^{\text {th }}$ responses were $2-3$ times larger than $1^{\text {st }}$ waves in that group. A comparison in the amplitude differences between the $1^{\text {st }}$ and $4^{\text {th }}$ responses, a measure of the strength of the recruiting response, was found to be significantly different for both MD and RE stimulation sites (Figure 2B and 2C). Morphology and latency measurements were similar to controls. 
To examine the evolution of this attenuation following SE, we examined recruiting responses at 2, 7 and 14 days following SE, all points prior to the onset of spontaneous seizures. At 2 days and 14 days following SE, we saw a decline in the response amplitudes, whereas we saw an increase at 7 days, (Table 2 and Figure 2). All responses in those groups were similar in both morphology and latency measurements.

In contrast to the decrease in response found in epileptic animals, recruiting responses in the kindled animals showed an increase in amplitude, producing the largest recruiting responses seen in this experiment. The change was significantly different compared to both epileptic and control animals (Table 2 and Figure 2). Again, latency measurements and morphology were similar to controls (Table 1).

\section{Seizure Recordings}

In order to determine if differential involvement of the PFC in seizure activity could have influenced group differences, specifically between kindled and epileptic animals, we recorded spontaneous seizures in epileptic animals as well as stimulated seizures in kindled animals in the PFC. In both groups we observed, although the exact seizure onset point was unclear, that there was a build-up in activity in the PFC within several seconds following hippocampal onset (Figure 3). In kindled animals, recruitment of the PFC in seizure activity occurred very early in the kindling process, usually by the first or second stimulation. The recording shown in Figure 3B and C was recorded after 5 stimulations, prior to the development of a motor component in the animal's seizure, although the PFC was involved by the second stimulation.

The observation of PFC involvement in both kindled and epileptic animals suggests that the presence or absence of seizure activity in the PFC in either model did not play a role in the differences seen between PFC responses in epileptic and kindled animals, as this area was consistently involved in hippocampal seizures in both groups.

\section{Anatomy}

It has been shown in previous studies that cell loss occurs in the MD and RE after status epilepticus, but not in our kindling model (Bertram et al. 2001). We used silver 
staining to determine if there was comparable cell loss in the PFC, as cell loss in this region could contribute to our results. We found that there was significant cell death specific to the MD and RE nuclei in the 7 Day animals, as expected (Figure 4). Positive staining was observed to be specific to the MD and RE nuclei within the midline thalamus, indicating that they were the nuclei most strongly affected by SE (see Figure 4 E-H). For each animal, cells unstained by silver were also seen in those nuclei, suggesting that cell loss in these nuclei was incomplete, and that there were still cells that could project to destinations such as the PFC.

By contrast, positive neurons in the PFC were rarely observed after SE (see Figure 4 A-D). Within the same coronal slices of SE rats, positive staining was observed in the piriform and olfactory nuclei, which are areas already known to be damaged by SE (Brandt et al. 2003). We found positive scattered staining in the PFC in only one of the five 7 Day post-SE animals, seen in layers I, III and VI.

\section{Discussion}

In this study, we found that there was a significant attenuation of thalamicallyevoked responses in the PFC of epileptic rats. The attenuation evolved over a period of weeks during the post-SE period. Differences in seizure activity in the PFC are not thought to be responsible for the change, as seizure activity was found in the PFC in both kindled and epileptic animals. Neuronal loss in the PFC was not found, although neuronal loss in the thalamus was confirmed. These findings have potential implications for prefrontal cortical function in TLE.

The recruiting response is a thalamically-induced cortical response that is specific to stimulation of the midline thalamic nuclei. Although the precise mechanisms of the recruiting response are largely unknown, the current theory is that the response is a result of the overlapping, or "non-specific" connectivity of the midline thalamus (Morison and Dempsey 1943, Verzuano et al. 1953, Schlag and Chillet 1962). The midline nuclei send monosynaptic projections to the entire medial PFC, with each nucleus overlapping in their termination fields, forming a diffuse and interconnected network. These projections 
terminate in layers I, III and VI of the PFC (Barbas et al. 1991, Hoover and Vertes 2007, Van der Werf et al. 2007, Negyessy et al. 2005). Reciprocal connections return from the PFC from layers II and V (Vertes 2004, Freedman et al. 1991). The theory is that the recruiting response is generated as this overlapping, reciprocal connectivity is able to incorporate and synchronize additional thalamic nuclei with each additional stimulation in a train. This is not to be confused with the similar "augmenting" response described in sensory and motor pathways, which is thought to be primarily cortically based (Herkenham 1986, Castro-Alamancos and Connors 1996). Also, it is possible that other structures in the limbic system that share connections with both the PFC and midline thalamus (such as the amygdala and hippocampus) may contribute to recruiting responses, but that possibility is, as yet, untested.

The changes we found in the PFC responses are likely related to the thalamic neuronal loss, but the mechanism is unclear at this time. The altered responses changed over time, which parallels the many changes that occur following an acute neuronal injury. Cell death and recovery is an evolutionary process which occurs in several brain regions in our epilepsy model (Cavalheiro et al. 1981, Ben-Ari et al. 1982, Dinocourt et al. 2003, Brandt et al. 2003). The process involves the loss of neurons, loss of synaptic input, local reinnervation and a spectrum of pre- and post-synaptic changes that alter individual and population excitability. The evolution of these changes have been studied extensively and in great detail in hippocampal and amygdalar circuits, among others, in epilepsy (Mangan and Bertram 1998, Sun et al. 2007, Sloviter et al. 2006, Kumar and Buckmaster 2006, Lehmann 2000). PFC neurons do not appear to die in our model of MTLE, but cell death in the midline nuclei likely causes a loss of first-line synaptic input, which, in turn, could lead to a level of deinnervation hypersensity (Koyama et al. 1993, Carlson et al. 2007, Viisanen et al. 2007, Iizuka et al. 2002) and alterations in postsynaptic channel and receptor expression. During the subsequent weeks, reinnervation takes place and post-synaptic properties stabilize and return to an equilibrium state, causing the response to decrease. This evolution timeline would explain the rise in recruiting response that takes place after 7 Days, and the subsequent decline. The exact mechanisms that cause these shifts in excitation are unknown. 
The basis for the change following kindling is also unclear. As mentioned, the process of cell dell that occurs in our epileptic model does not occur in our kindling model. (Racine 2002, Represa 1993). Changes in kindled animals may include postsynaptic alterations as well as other mechanisms which allow for a better recruitment of the other thalamic nuclei that contribute to the recruiting response.

The finding that the peak latencies were not significantly different among groups in this study reflects some minor variance in the geometry of the peak from rat to rat, the slight variation in rise time to high and low amplitude peaks, and may also reflect the differences in post-synaptic properties to which we have alluded. The lack of any significant difference between onset latencies among groups suggests that latency is not a truly variable factor in observations.

The effect that the alteration in responses has on the normal function of the PFC, if any, is also unclear. The PFC is involved in various memory functions, including autobiographical, spatio-temporal and working memory (Macrae et al. 2004), as well as emotional processing, executive functioning and decision-making (Price 2005, Barbas et al. 1995). Lesions in the PFC have given mixed but interesting results depending on the animal and the method used (Deacon et al. 2003, Lacroix et al. 1998), and abnormalities in the PFC have been implicated as underlying factors in some psychiatric disorders, such as schizophrenia (Mitelman et al. 2005). Although the deficits in learning and memory that have been observed in patients with TLE (Glowinski 1973, Viskontas et al. 2000, Voltzenlogel et al. 2006) have been rightly attributed to cellular damage in the hippocampus and other limbic structures, our results suggest that the effects of that damage may extend into the physiology, and perhaps functionality, of structures like the PFC. This may even be the case in the kindled animals, in which some memory deficits have been suggested (Hanneson et al. 2001). More detailed studies on this subject will be difficult, as it will not be easy to attribute a specific memory deficiency to the PFC or any other limbic structure.

In conclusion, we have found an attenuation in PFC responses to midline thalamic stimulation in epileptic rats, suggesting an impairment in their connection. This attenuation evolves following thalamic cell injury incurred by status epilepticus and may 
be attributable that cell loss. Other factors, including inputs to the PFC from other structures and possible pre- and post-synaptic modifications may also contribute to the change in response. It is possible that this physiological attenuation may be an indication of some form of functional deficiency in the PFC, with implications for its involvement with learning and memory processes. These data reinforce our current understanding that a variety of higher-order functions require a complex circuitry, incorporating diverse groups of brain structures.

\section{Acknowledgements}

This study was funded by NIH Grant NS25605. We thank John Williamson for his excellent technical support. 


\section{$\underline{\text { Tables }}$}

\begin{tabular}{|c|c|c|c|}
\hline \multirow{2}{*}{\multicolumn{4}{|c|}{$\begin{array}{l}\text { A. Electrode Depths (in } \\
\text { mm) } \\
\quad \text { MD Stimulation }\end{array}$}} \\
\hline & & & \\
\hline Stimulation & $5.1 \pm 0.1$ & $5.2 \pm 0.1$ & $5.3 \pm .1$ \\
\hline Recording & $2.6 \pm 0.2$ & $2.2 \pm 0.2$ & $2.7 \pm .2$ \\
\hline \multicolumn{4}{|l|}{$\underline{\mathrm{RE} \text { Stimulation }}$} \\
\hline Stimulation & $6.5 \pm 0.2$ & $7.0 \pm 0.1$ & $6.7 \pm 0.1$ \\
\hline Recording & $3.1 \pm 0.2$ & $2.8 \pm 0.4$ & $3.1 \pm 0.2$ \\
\hline \multicolumn{4}{|l|}{ B. Latencies (in ms) } \\
\hline \multicolumn{4}{|l|}{ Onset Latency } \\
\hline MD stim & $11.6 \pm 2.1$ & $9 \pm 1.4$ & $11.1 \pm 1.9$ \\
\hline RE stim & $11.3 \pm 1.7$ & $8.5 \pm 1.7$ & $7.6 \pm 1.0$ \\
\hline \multicolumn{4}{|l|}{ Peak Latency } \\
\hline MD stim & $27.11 \pm 3.1$ & $21.72 \pm 1.1$ & $25.29 \pm 2.8$ \\
\hline RE stim & $26.36 \pm 3.4$ & $21.13 \pm 1.5$ & $22.23 \pm 1.0$ \\
\hline
\end{tabular}

Table 1: Maximum Response Depths and Latencies in Control, Epileptic and Kindled Animals.

(A) Average depths for recording and stimulating electrodes at which maximal recruiting responses were obtained, in $\mathrm{mm}$ below surface. (B) Latencies for both the onset and peak of the response waveforms at maximum amplitude, in $\mathrm{ms}$ following the stimulation. Differences between groups were not statistically significant in either $A$ or $B(P>.05$ by t-test).

\begin{tabular}{|c|c|c|c|c|c|c|}
\hline \multicolumn{7}{|c|}{ Absolute Amplitudes (mV) } \\
\hline Stim \# & Control $(n=12)$ & 2 Day (n=5) & 1 Week $(n=6)$ & 2 Week $(n=6)$ & Epileptic $(\mathrm{n}=6)$ & Kindling $(\mathrm{n}=6)$ \\
\hline \multicolumn{7}{|c|}{ Max Responses-MD } \\
\hline Stim 1 & $3.81 \pm 2.23$ & $3.68 \pm 1.26$ & $3.38 \pm 1.69$ & $4.62 \pm 1.27$ & $2.60 \pm 0.54$ & $6.43 \pm 1.26 *$ \\
\hline Stim 4 & $13.39 \pm 6.72$ & $9.18 \pm 2.17$ & $18.13 \pm 3.52 \dagger$ & $8.73 \pm 1.84$ & $5.25 \pm 0.50 *$ & $24.28 \pm 2.81 * \dagger$ \\
\hline \multicolumn{7}{|c|}{ Max Responses-RE } \\
\hline Stim 1 & $5.12 \pm .827$ & $4.58 \pm 1.185$ & $5.52 \pm 2.37$ & $5.15 \pm 8.71$ & $1.93 \pm 0.61 *$ & $8.85 \pm 1.60 \dagger$ \\
\hline Stim 4 & $15.94 \pm 1.83$ & $10.45 \pm 2.94$ & $18.8 \pm 4.50 \dagger$ & $10.38 \pm 13.73$ & $6.52 \pm 1.02 *$ & $29.35 \pm 5.26 \dagger^{*}$ \\
\hline
\end{tabular}


Table 2- Comparison of Max Responses

Average amplitudes (in $\mathrm{mV}$ ) of the $1^{\text {st }}$ and $4^{\text {th }}$ response waves to MD and RE stimulation for each group of animals studied, with their standard errors.

Differences across groups were significant $(p<.001$ by ANOVA, $d f=40, F=6.95$ for $\mathrm{MD}, 5.57$ for RE). Post-hoc SNK method revealed pairwise differences. * indicates $\mathrm{P}<.05$ compared to control, $\dagger$ indicates $\mathrm{P}<.05$ compared to epileptic.

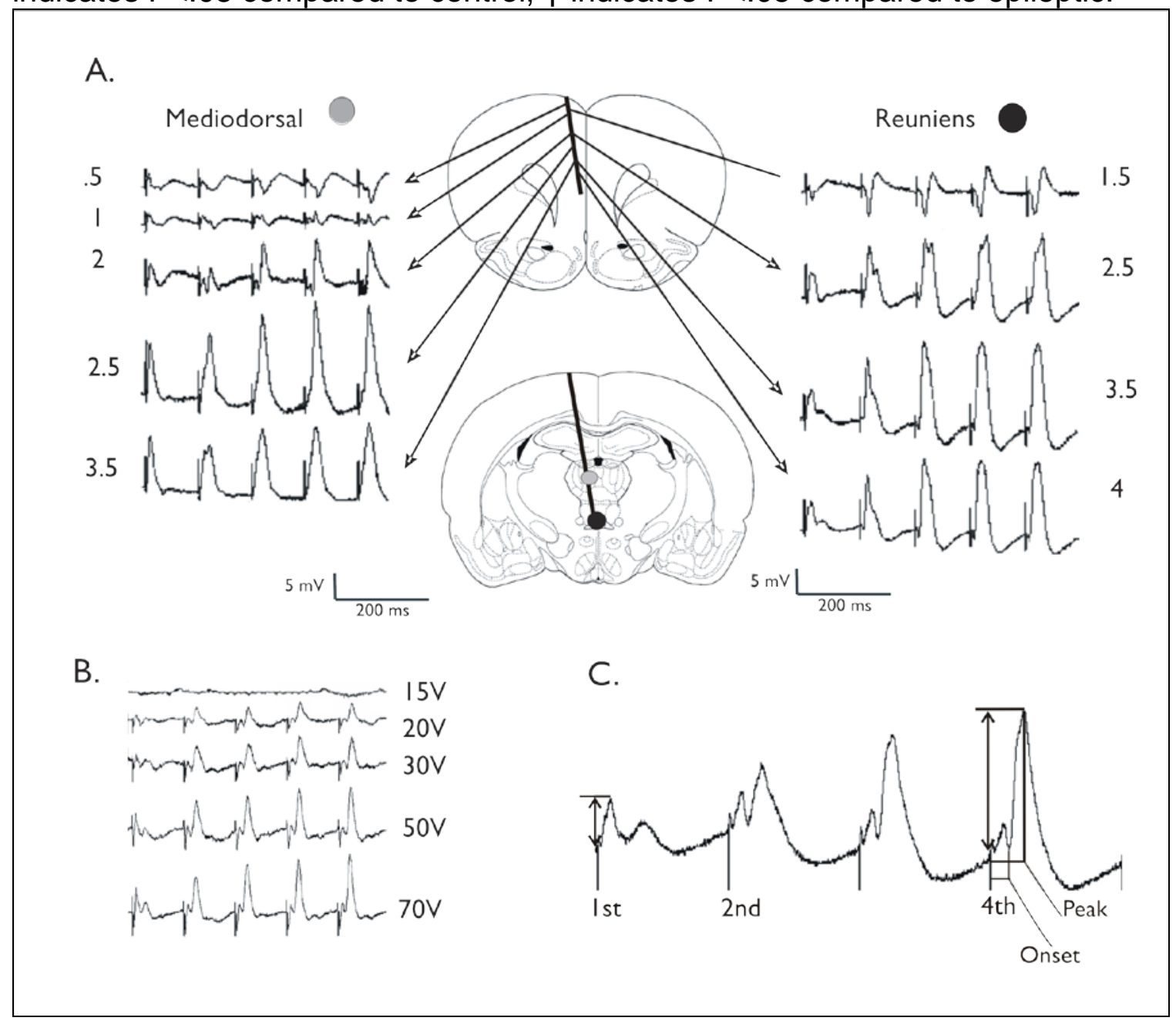

Figure 1: Figure 1- Recruiting Responses

(A) Typical responses in the medial PFC to midline thalamic stimulation in control animals. Responses on left were elicited from MD stimulation (gray circle), while those on the right were elicited from RE stimulation (black circle). Arrows indicate depths at which responses were obtained. The recording electrode was moved from 1-4 $\mathrm{mm}$ below the cortical surface in order to identify areas of maximal response (see Table 1). Notice that the amplitude of the response increases until the $4^{\text {th }}$ stimulation and then plateaus. (B) Responses recorded at stimulation intensities ranging from 15-70 V. Maximal responses did not significantly increase with higher stimulation intensities after 50V. (Images of sections from Paxinos and Watson 1984). (C) Demonstration of how parameters were measured. $1^{\text {st }}$ and $4^{\text {th }}$ response wave amplitudes were 
measured from the baseline previous to the stimulation, and then compared. Latencies were measured from the stimulation itself. Onset latency was measured to the start of the wave, and peak latency was measured to the highest point of the positive recruiting response.

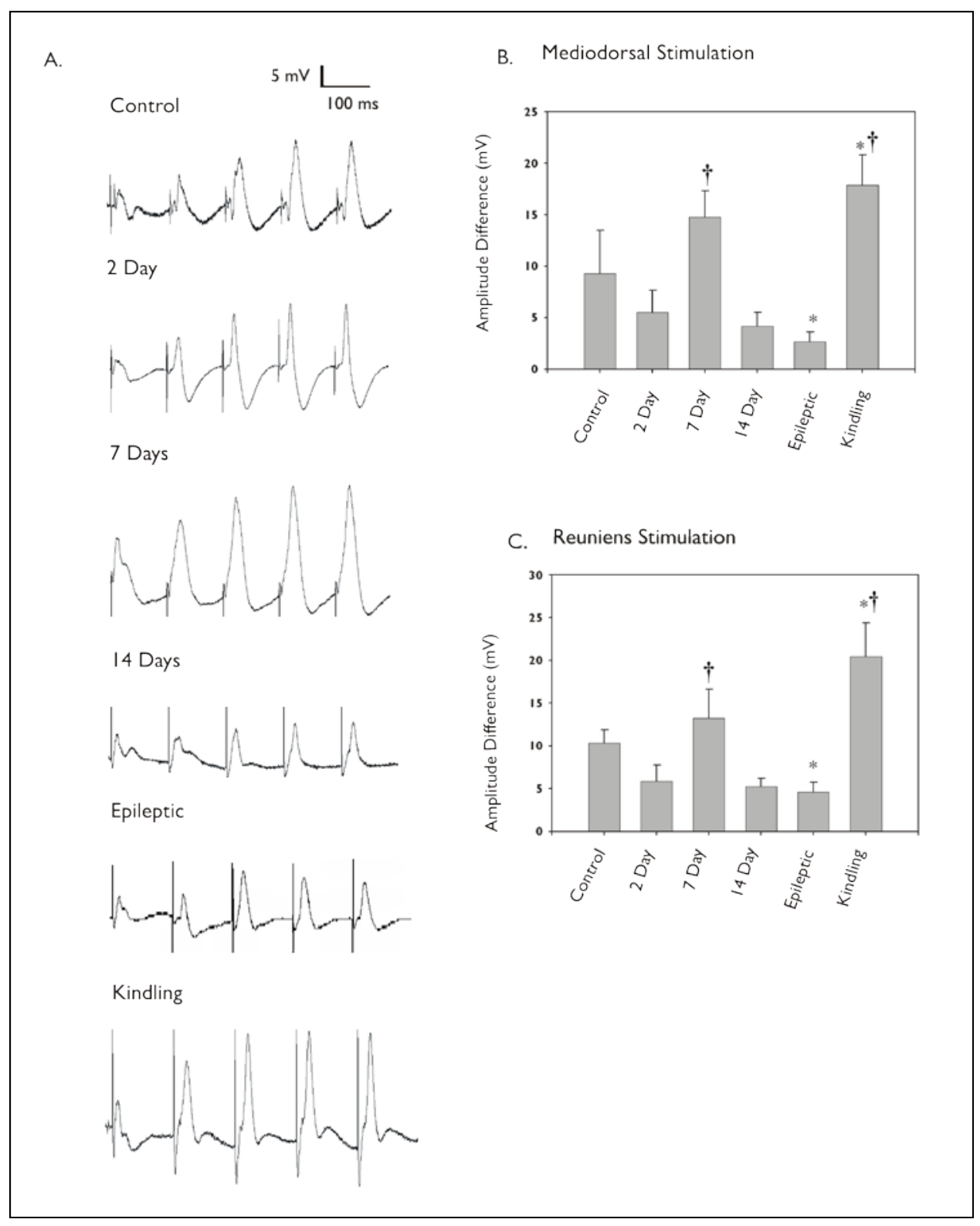

Figure 2- Time Course and Kindling Responses

(A) Representative maximal responses to RE stimulation from animals at different time points following status epilepticus. ( $B$ and $C$ ) Comparisons of the amplitude differences between the $1^{\text {st }}$ and $4^{\text {th }}$ response waves to MD (B) and RE (C) stimulation from each of the 6 different groups studied, demonstrating changes in the recruiting response. Notice the increase after 7 days, followed by a decline to epileptic. Also notice the increase in the kindled animals compared to both controls and epileptics. Differences across groups were significant $(p<.001$ by ANOVA, $\mathrm{df}=39, \mathrm{~F}=6.95$ for MD, 5.57 for RE). Post-hoc Student-Newman-Kools (SNK) method revealed 
pairwise differences. * indicates $\mathrm{P}<.05$ compared to control, $\uparrow$ indicates $\mathrm{P}<.05$ compared to epileptic.

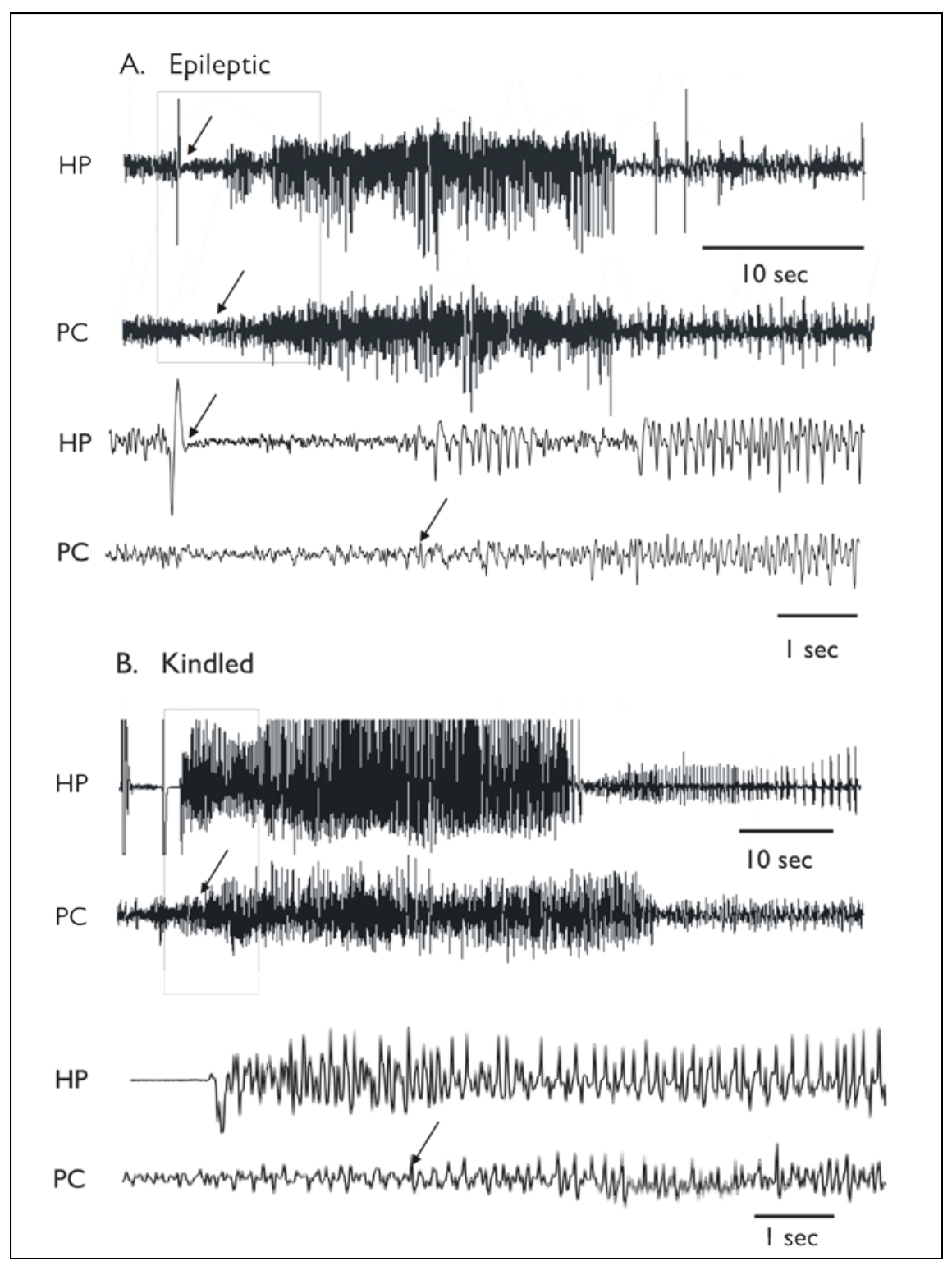

Figure 3-Seizures in the Medial PFC in both Epileptic and Kindled Rats

(A) Seizure recording from an epileptic rat implanted with electrodes in CA1 of the hippocampus and in the medial prefrontal cortex. (B) Recording from a kindled animal with electrodes in the same regions as those in the epileptic animal shown. Arrows indicate point of seizure onset. Recordings indicate that the PFC may be an early target in the spread of limbic seizures 
Control

A

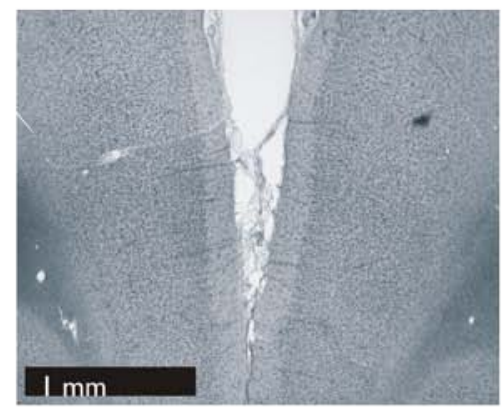

C

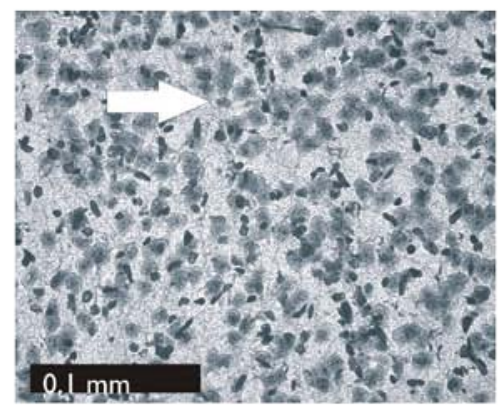

$\mathrm{E}$

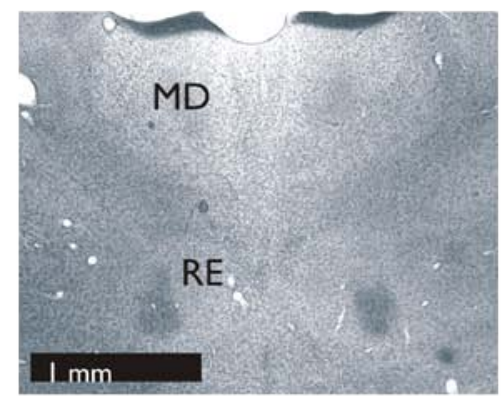

G

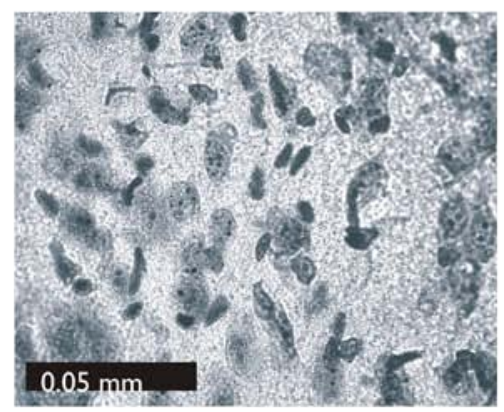

B

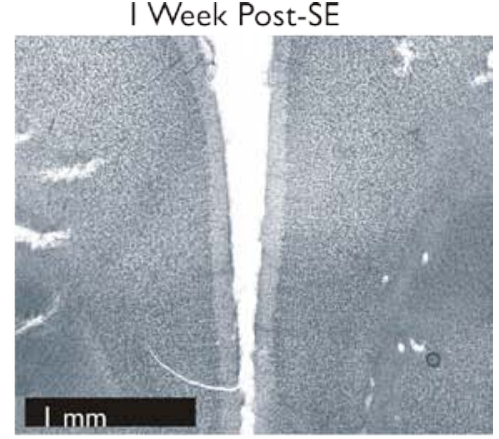

D

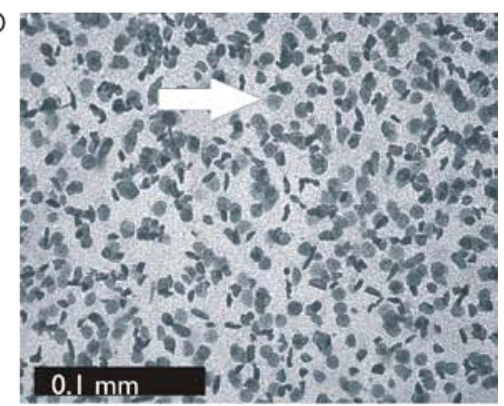

F

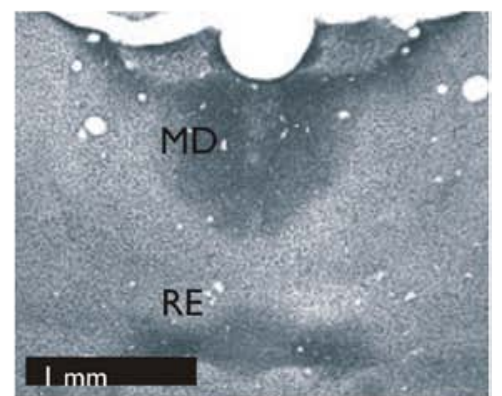

$\mathrm{H}$

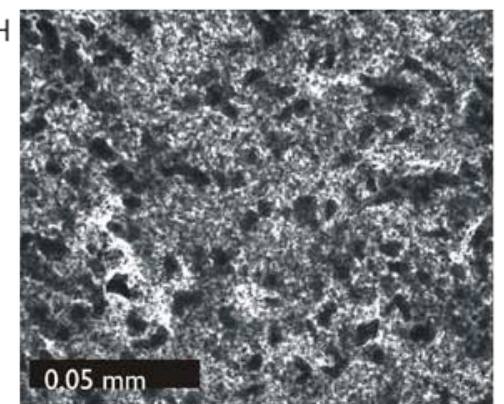

\section{Figure 4- Silver Staining of Prefrontal and Thalamic Areas}

(A) and (B) are from a Control and 1 Week post-SE animals, respectively, at the PFC at $4 \mathrm{X}$ magnification. (C) and (D) show layer III of each at 40X. No identifiable change was observed in the prefrontal staining. (E) and $(F)$ are from the same animals. Note the pronounced staining of the MD and Re in the 1 week animal in $(F) .(G)$ and $(H)$ are 100X images of the MD of each. Note the increased dark staining due to positive staining of the 1 Week animal in $(F)$ and $(H)$. Scale bars in $A, B, E, F=1 \mathrm{~mm}, C$ and $D=.1 \mathrm{~mm}, G$ and $H=.05 \mathrm{~mm}$. 


\section{Chapter III}

Excitatory Amplification Through Divergent-Convergent Circuits: The Role of the Midline Thalamus in Limbic Seizures

Summary

Introduction: The midline thalamus is an important component of limbic seizures. Although the anatomic connections and excitatory influences of the midline thalamus are well known, its physiological role in limbic seizures is unclear. We examined the role of the midline thalamus on two circuits that are involved in limbic seizures: (a) the subiculum-prefrontal cortex (SB-PFC), and (b) the piriform cortexentorhinal crextex (PC-EC).

Methods: Ev ked field potentials for both circuits were obtained in anesthetized rats, and the :ikely direct monosynaptic and polysynaptic contributions to the responses were identified. Seizures were generated in both circuits by $20 \mathrm{~Hz}$ stimulus trains. Once stable seizures and evoked potentials were established, the midline thalamus was inactivated through an injection of tetrodotoxin (TTX), and the effects on the evoked responses and seizures were analyzed.

$\underline{\text { Results: }}$ Inactivation of the midline thalamus suppressed seizures in both circuits. Seizure suppression was associated with a significant reduction in the late thalamic component but no significant change in the early direct monosynaptic component. Injections that did not suppress the seizures did not alter the evoked potentials.

Conclusions: Suppression of the late thalamic component of the evoked potential at the time of seizure suppression suggests that the thalamus facilitates seizure induction by extending the duration of excitatory drive through a divergent- 
convergent excitatory amplification system. This work may have broader implications for understanding signaling in the limbic system.

\section{Introduction}

The mechanisms by which seizures initiate and spread within the limbic network remain unclear. Defining the role of the different regions of the limbic network in seizure activity is important for determining the key components that may be targets in clinical therapies.

One component that is critical to limbic seizures is the midline thalamus. This region has extensive, overlapping, reciprocal connections to much of the limbic network (Ray et al. 1992, Van der Werf et al. 2002, Berendse and Groenewegen 1991, Hoover and Vertes 2007), and experimental evidence has shown that these nuclei play a major role in seizure initiation and spread (Cassidy and Gale 1998, Bertram et al. 2001, 2008). While the anatomic and experimental evidence is clear, the actual physiological role of the midline thalamus in limbic seizures has not yet been defined. The complexity of the limbic network, in which each region receives and sends connections to many others, makes it difficult to isolate the contributions of any single region, but such studies are important to understanding how seizure activity operates within the network.

Because the thalamus receives afferents from many limbic regions, and sends strong, excitatory efferents to many of the same regions, a framework exists for an accessory excitatory pathway through the thalamus, a "divergent-convergent circuit”, capable of enhancing the direct region-to-region monosynaptic excitatory connections throughout the limbic network. Two such possible pathways are (a) the subiculum (SB) to the medial prefrontal cortex (PFC), and (b) the piriform cortex (PC) to the entorhinal cortex (EC). In both cases, there is a monosynaptic connection that forms the primary direct path, as well as a secondary, disynaptic or polysynaptic connection through the thalamus. The pathway through the midline thalamus may provide additional excitatory input that has the potential to amplify the direct monosynaptic signal. During a seizure, this secondary thalamic influence may help drive seizure activity in both pathways. 
In this study, we hypothesized that the midline thalamus provides a second excitatory input that helps to drive seizure activity in limbic circuits. To evaluate the physiological influence of thalamic inputs on activity in the limbic circuits, we evoked field potentials from both SB-PFC and PC-EC pathways. We identified which components of these field potentials were influenced through polysynaptic pathways and then evaluated how these components changed with pharmacological inactivation of the thalamus. Our results suggest a role for the thalamus in initiating and spreading seizures in limbic circuits through divergent-convergent amplification of excitatory drive.

\section{Materials and Methods}

\section{Animal Preparation and Electrode Placement}

All animals were used in accordance with protocols approved by the Animal Care and Use Committee at the University of Virginia. All stereotactic coordinates were derived from the rat brain atlas of Paxinos and Watson (1998). Young adult male Sprague-Dawley rats (250-300 g) were used for all experiments.

Rats were anesthetized with urethane (1.2g/kg, i.p.) and placed in a multi-armed stereotactic frame (Kopf Instruments, CA, USA). The skull was exposed and holes were drilled for electrode placement. Electrical stimulation was performed with bipolar, insulated, twisted pair stainless steel electrodes with tip separation of approximately 1 $\mathrm{mm}$, and responses were recorded through a pulled glass micropipette with an approximate $1 \mathrm{M} \Omega$ tip resistance, filled with $0.9 \%$ saline and $1 \%$ Fast Green dye. The recording electrode was connected to the system through a silver-silver chloride wire. Following the experiment, the animal was killed while still under anesthesia and the brain was placed in $4.0 \%$ paraformaldehyde. Frozen sections confirmed electrode placements. Electrode positions were then correlated to the success of the experiment.

\section{Electrode Placement: for Subiculum to Prefrontal Cortex (SB-PFC)}

The subiculum has a monosynaptic connection to the medial prefrontal cortex, and there is a potential disynaptic pathway through the medial dorsal thalamic nucleus. A 
stimulating electrode was placed in the dorsal SB (from bregma, in mm): AP -6.3 to 7.0, ML 3.8 to 4.0, DV -2.5 to -3.0 . The recording electrode was placed in the medial ipsilateral PFC: AP +2.7 to 3.2, ML 0.5, DV 2.0-3.5, 2.0 angle lateral to vertical (Figure 1A)

Electrode Placement: for Piriform Cortex to Entorhinal Cortex (PC-EC)

The piriform cortex has a major monosynaptic input to the entorhinal cortex. In addition, there is a potential disynaptic pathway through the medial dorsal nucleus. The stimulating electrode was placed in the anterior PC: AP +1.8 to 2.5 , ML 4.0 to 4.5 , DV -6.5 . The EC recording electrode was placed AP -6.8 to -7.3, ML 4.5 to 5.0, DV -6.3 to -7.0 (Figure $1 \mathrm{~A})$.

\section{Electrode Placement: Thalamus}

Stimulation or recording electrodes were placed in the ipsilateral mediodorsal nucleus: AP -1.8 to 2.3 , ML 0.5 , DV -4.8 to $-5.5,5.0^{\circ}$ lateral to vertical.

\section{Stimulation Protocols}

Acquisition: Stimulus trains were delivered using a Winston timer connected to a constant-voltage stimulus isolation unit (SIU). Responses were recorded using an Axon Smartprobe connected to a Cyberamp. Responses were digitized at a rate of $10 \mathrm{kHz}$ through a Digidata 1440 digitizer and analyzed using Axoscope 8.0 software (Molecular Devices, CA, USA). Responses were filtered with a lowpass filter of $1 \mathrm{kHz}$ and highpass filter of $10 \mathrm{~Hz}$.

Evoked responses: Because we are interested in the potential thalamic component of the evoked responses, it was necessary to ensure that we obtained a maximal response that resulted from activation of the mediodorsal nucleus. Because direct stimulation of the mediodorsal nucleus results in increasing response amplitudes in the target region with repeated stimulation (recruiting response) we used a standard recruiting response stimulation protocol (Sloan and Bertram 2009, Dempsey and Morison 1942, Verzeano et 
al. 1953, Bazhenov et al. 1998). Stimulation consisted of $0.17 \mathrm{~ms}$ pulses at an interstimulation interval (i.s.i.) of $120 \mathrm{~ms}$ ( 8.3 Hz). Responses were evoked by delivering stimulus trains to the SB, PC or thalamus. A minimum of five trains of six pulses were delivered and averaged every ten minutes. Electrode depths were adjusted to obtain maximal responses (Figure 1). Maximal responses were obtained by increasing stimulus intensity stepwise until the response amplitudes plateaued or until an intensity of 70V was reached (maximal current: 2.3 to $4.7 \mathrm{~mA}$, at a typical stimulating electrode resistance of $15-30 \mathrm{k} \Omega$ ). If the responses had not plateaued at $70 \mathrm{~V}$, or did not show the standard morphology, we found that one or both electrodes were off target and such data were discarded.

Measurements of evoked response amplitude and latency of the individual peaks were made with reference to the baseline prior to the stimulation pulse for amplitude and from onset of the stimulation pulse for latency. Because the thalamic component of the evoked potential increased with each repeated stimulation pulse before plateauing, all field potential measurements were made using the fourth stimulation. For the graphs in Figure 5, our analysis also included the differences in amplitudes between peaks within the waveform as a means of comparing changes in early and late components. Statistical analyses were performed using SigmaStat 3.0 software. Differences in average amplitudes and latencies before and after TTX injection, as well as LTP-induced changes, were compared using paired t-test, while comparisons of amplitude differences between early and late components used student's t-test. Significance was set at the $\mathrm{p}<.05$ level.

Seizure Induction: Seizure afterdischarges were elicited by a $20 \mathrm{~Hz}$ train consisting of monophasic pulses of 0.17 ms duration for five seconds at the same intensity that was used for the evoked potentials (range 50-70V, up to $4.7 \mathrm{~mA}$ ). Stimuli were given at standard ten minute intervals immediately following each evoked response stimulus session. Seizures were identified as large, continuous spike bursts beginning at the end of the stimulus. Seizure termination was determined by the end of the last regularly occurring spike burst. For each experiment, a minimum of ten stimulus trains were required to induce seizures of a consistent duration. Once a stable seizure duration was 
achieved, defined as a minimum of three seizures of consistent duration of a minimum of 10 seconds duration with less than $30 \%$ variation between them. This seizureinduction method allows for controlled initiation of seizures, allowing for accurate timing for drug injection protocol.

\section{Identification of Components of Evoked Potentials}

Because these field potentials are complex evoked responses that are composed of postsynaptic potentials that are driven by mono-, di- and potentially polysynaptic sources, it is necessary to estimate the likely time windows in which different inputs are likely to have their contribution to the overall field potential.

Input/Output Protocol: As monosynaptic components of a field response are more likely to appear at lower stimulation intensities, we performed an input/output protocol to determine which waveform components appeared at lower stimulation intensities, and which appeared as the stimulation intensities were increased. The stimulations were initiated with a train of $10 \mathrm{~V}$ pulses with 5 trains averaged per intensity. The intensity was increased stepwise by $10 \mathrm{~V}(0.33 \mathrm{~mA})$ until a plateau was achieved, or to a maximum of $70 \mathrm{~V}(4.3 \mathrm{~mA})$. This protocol ensured that maximal responses were obtained for each animal.

Long-term potentiation (LTP): Because LTP is generally considered a monosynaptic process, we induced LTP in both circuits to help determine which components were more likely monosynaptic. We used previously established protocols (Laroche et al 1990, Degenetais et al. 2003, Kawashima et al. 2006) Recordings of two pulses (120 i.s.i.) were made every three minutes at $50 \%$ of the maximal stimulus intensity. LTP induction was achieved through 12 bursts of $200 \mathrm{~Hz}$ trains at maximum voltage, with each train lasting for $250 \mathrm{~ms}$ with a 1 second inter-train interval. Two LTP induction sessions were applied, with ten minutes rest between them. Recordings were continued every three minutes for a half-hour following the second train, after which pulses were recorded every five minutes for another twenty minutes. A minimum of five trains, with a ten 
second inter interval between trains, was averaged for each recording. We analyzed the significant peaks, early and late, to determine which ones had changed and how. The short trains and sub-maximal stimulations were used in order to isolate the monosynaptic components and reduce polysynaptic components as much as possible.

Polysynaptic Component Construct: To identify a minimum latency for the thalamic polysynaptic component to appear in the field potentials, we recorded from two points in the potential disynaptic pathway in both circuits (e.g., SB-Thalamus and Thalamus-PFC) and used these data to estimate a potential time window (earliest likely time of occurrence) for a contribution of a disynaptic response from the thalamic pathway. By temporally aligning the responses from the two sequential pathways in and out of the medial dorsal nucleus, we estimated an approximate minimum latency for thalamic involvement in our evoked potentials.

\section{TTX Drug Delivery}

Prior to injection of TTX, all animals had to have a baseline seizure duration and evoked potential morphology, as described above, that was consistent for 30 minutes after the placement of the canula into the thalamus, but prior to injection.

To deliver drugs to the thalamus, a 25 ul Hamilton syringe was loaded with either $0.1 \mathrm{mM}$ tetrodotoxin (TTX) or $0.9 \%$ saline solution for vehicle control. The tip of the syringe was placed into the ipsilateral mediodorsal nucleus (AP -1.8 to 2.3, ML 0.5, DV 4.8 to $5.5,2.0^{\circ}$ medial to vertical). Confirmation of canula placement was included during confirmation of electrode placement. Previous work has shown that injections of TTX outside of the midline thalamic nuclei have no effect on seizures, so proper placement was judged initially by seizure suppression. A rest period of ten minutes was allowed after the injection prior to resuming stimulations to permit sufficient spread of the drug and to minimize the possibility of mechanical effects from the injection. The standard TTX injection was $0.75 \mu \mathrm{l}(0.1 \mathrm{mM})$, released slowly over three minutes. An effect seen only after 20 minutes post-injection was interpreted as being an injection outside of the target zone. Seizure suppression persisted for 40 to 80 minutes before 
recovering. Seizure duration had to be reduced by 75\% from baseline for longer than 20 minutes to be considered a successful suppression.

If either the electrodes or canula were afterwards found to be outside of their designated regions, the data were discarded.

\section{Results}

\section{Evoked Field Potentials}

Trains of evoked potentials with consistent morphologies were recorded from both the Subiculum to Prefrontal Cortex (SB-PFC) $(n=6)$ and Piriform Cortex to Entorhinal Cortex (PC-EC) ( $\mathrm{n}=5$ ) circuits (Figure 1). Because the morphology of the wavefroms varied by recording depth, electrodes were adjusted to ensure a standard morphology of the responses (Figure 1E). The average depth of recordings used in the PFC was $2.5+/-0.5 \mathrm{~mm}$, and the average depth used in the EC was $6.3+/-0.3 \mathrm{~mm}$.

Both sets of field potentials were complex, containing multiple positive and negative peaks at maximal stimulus intensity. Amplitudes of all peaks increased in complexity between the $1^{\text {st }}$ and $4^{\text {th }}$ stimulation of the train. The $4^{\text {th }}$ stimulation of the train was used for analysis because all peak amplitudes had plateaued by that point, and because the likelihood of a disynaptic pathway through the thalamus being incorporated into the response was increased (see Methods). In the case of the SB-PFC responses, the responses consisted of two positive (P1 and P2) and two negative (N1 and N2) waves. In the case of the PC-EC circuit, the field potential included a $3^{\text {rd }}$ positive wave (P3).

When stimulus intensity was increased stepwise, all peaks in the responses from both circuits increased in amplitude with stronger stimulus intensities (Figure 2). In the SB-PFC responses, P1 did not change significantly, and N1 plateaued by the time it reached $75 \%$ stimulation. P2 and N2 did not plateau until the max stimulation. In the PC-EC, the P1, N1 and P3 increased significantly in amplitude until they plateaued at max stimulation. Maximal stimulus intensities were used to ensure that all peaks were present at their maximum amplitudes. 
Confirmation of Early Monosynaptic Component: LTP

Prior to attempting to isolate polysynaptic components through the thalamus, the monosynaptic components had to be identified. The changes that occurred in the responses as stimulus intensities were increased, in which earlier peaks required less intensity then later peaks, suggested that earlier peaks are more likely to represent direct monosynaptic inputs (Figure 2). To obtain further evidence that the early components represented a monosynaptic connection, we used long-term potentiation (LTP), which is generally considered to be a monosynaptic phenomenon.

In order to minimize the influence of a potential thalamic recruiting response or LTP effects through polysynaptic circuits, we stimulated at half-maximal intensity and reduced the stimulus train to a paired pulse. Because of these changes in stimulation parameters, the responses were less robust compared to the maximal multiple pulse stimulus train responses. The $2^{\text {nd }}$ response of the pair was evaluated for the potential effect of the potentiating stimulation. We emphasize that peak latencies in these responses, due to the low stimulation intensity and the lack of a train, are not precise correlates to peaks from the control responses described (see Figure 2 for differences between maximal and half-maximal stimulations).

The LTP protocol caused an increase in peak amplitudes in both sets of responses (Figure 3). In the SB-PFC circuit ( $\mathrm{n}=5)$, at half-maximal stimulation, the N1 with a latency of $21 \mathrm{~ms}$ increased in amplitude by approximately 100\% after potentiation (Figure 3) and didn't change for the duration of the experiment (35 min). In the PC-EC circuit ( $\mathrm{n}=7)$, the $\mathrm{N} 2$ at a latency of $32 \mathrm{~ms}$ increased by approximately 40\% (Figure 3B) and held for the duration of the experiment. Both of these peaks occur within the range considered to form the "early" component of the response, and the experiment suggests that these peaks primarily represent the monosynaptic input. The lack of change in P1 in both circuits suggests that the peak may represent an axon volley, rather than a synaptic response. The effect of the LTP protocol and the latencies of the affected peaks correlate with previous findings in these circuits (Laroche et al. 1990, Degenetais et al. 2003, Kawashima et al. 2006, Hamam et al. 2007). 


\section{Composition of Field Potentials}

In order to estimate approximate time windows for the appearance of a possible thalamic component to the overall responses, we needed to identify the minimum time required for thalamically-mediated post-synaptic potential to appear.

To determine a minimum latency for a signal passing through the thalamus, we recorded evoked responses in the medial dorsal nucleus after stimulation in either the SB or PC. We also stimulated in the mediodorsal nucleus and recorded in the PFC or EC. We then combined the two sets of data to estimate the transit time of a signal from the primary stimulation site (SB or PC) through the thalamus and from the thalamus to the recording site in a circuit that includes at least two synapses. Responses were generated by single stimulations. The first peak occurring after $6 \mathrm{~ms}$ in each of the responses was used for the alignment. The results of this alignment are shown in Figure 3. Key aspects of the responses include:

For SB-Thal-PFC disynaptic pathway

Subiculum to Thalamus: Responses were very short and of low amplitude, consisting of a sharp early peak, and then a larger negative peak which gradually recovers to baseline.

Thalamus to Prefrontal Cortex: Responses have one primary peak, sometimes followed by a secondary peak, followed by a gradual recovery. These responses are large, and, were they stimulated in a train, would greatly increase in amplitude over the course of a train (Bertram and Sloan 2009).

For PC-Thal-EC disynaptic circuit

Piriform Cortex to Thalamus: Begins with a short negative, followed by a positive peak, followed by a negative wave that recovers. The positive peak is considered the primary signal. 
Thalamus to Entorhinal Cortex: Consists of a sharp early peak, a small negative peak, a large positive peak and a deep negative wave that recovers gradually. The early peak is probably an axonal volley.

By adding the latencies of the first peak in each recording in the disynaptic pathway, we came to an estimated minimum latency for a thalamic component to appear in the primary recording site, as shown in Figure 4. These results, combined with the LTP and stimulus intensity results (in which a greater stimulus intensity was required to generate later peaks), as well as the thalamus-like augmentation of late responses over the course of the train, we considered the "early" component of the responses (before $35 \mathrm{~ms}$ ) to primarily contain the monosynaptic direct signal and the "late" component (after 35 ms) to be multisynaptic and to contain the contribution of the thalamus.

\section{Seizures and Effect of Thalamic Inactivation}

Seizures were reliably induced from both initiating sites by $20 \mathrm{~Hz}$ stimulations (Figure 5). Seizures were defined as repetitive bursts of spikes. Seizure termination was determined to occur after the last regular burst discharge, followed by suppression of the EEG amplitude compared to the prestimulation baseline duration and was defined as the interval between the end of stimulation and the end of the last burst discharge. Baseline seizure durations were between 15 and 25 seconds for both circuits, although PC-induced seizures tended to be shorter than those induced in the SB.

Injection of TTX into the midline thalamus caused a reduction or complete suppression of seizure activity in both circuits (Figure 5). A seizure considered suppressed when the seizure duration was reduced by at least 70\% from the baseline duration for at least 30 minutes, if any seizure was present. The effect of the drug lasted, on average, 40-80 minutes, during which the seizures gradually recovered. This process of seizure reduction and recovery has been demonstrated previously (Cassidy and Gale 1998, Bertram et al. 2008). We considered a successful reduction in seizure activity as a marker indicating that the canula was correctly placed in the thalamus and that inactivation of the thalamus was successful. Injections of saline in the mediodorsal 
nucleus showed a brief effect on field potentials, but this effect never lasted longer than ten minutes. Failed injections caused no change in either seizure duration or evoked potentials (Figure 7B).

\section{Effects of Thalamic Inactivation on Evoked Potentials}

All injections of TTX into the midline thalamus that were sufficient to cause a reduction of seizure activity also altered evoked field potentials in both circuits, particularly in the late components in both sets of responses (Figure 6). In the case of the SB-PFC circuit, all peaks except P1 were affected by the drug, but peaks in the late component were the most significantly reduced (Late component, N2-P2, reduced from $1.94+/-0.30 \mathrm{mV}$ to $0.50+/-0.10 \mathrm{mV}$ ). In the case of the PC-EC circuit, the P3 peak was significantly reduced, while the other peaks remained close to control levels (P3 reduced from $1.44+/-0.25 \mathrm{mV}$ to $0.30+/-0.13 \mathrm{mV}$ ). Latencies remained similar to control measurements. Injection of TTX outside of the borders of the mediodorsal nucleus did not yield any changes to seizure duration or evoked potential amplitudes (Figure 7C). Changes to the evoked responses persisted for the same amount of time as seizure suppression, and recovered as seizures recovered.

\section{Discussion}

In this paper, we used a combination of evoked field potentials and kindled seizures in two different limbic pathways to identify a role for the thalamus in the initiation and spread of limbic seizures. Evoked potentials were reliably obtained by stimulating in the subiculum or piriform cortex and recording in the prefrontal or entorhinal corticies, respectively. Both pathways had direct, monosynaptic connections as well as a potential indirect, polysynaptic pathway through the thalamus. Pharmacological blockade of the mediodorsal nucleus of the thalamus suppressed seizures generated in both pathways, and concurrently reduced the late polysynaptic

components of the evoked potentials. That the loss of the polysynaptic pathway through the thalamus is associated with attenuated seizure activity and loss of the late component 
of the field potentials suggests that thalamically-mediated excitation may provide an additional excitatory drive that prolongs the duration of excitation.

The thalamus has pathways that may be critical in facilitating communication between different regions throughout the brain. A divergent-convergent circuit is one in which two pathways emerge from a starting point and reconvene at the same target. Although the functional value of the D-C circuits discussed has not yet been established, they have the potential function of prolonging depolarization at the target by sending two time-staggered excitatory signals, thereby amplifying the signal. For example, in sensory cortex, afferent neurons extending from layer $\mathrm{V}$ have been shown to drive a circuit through the thalamus that will reinforce direct cortical-cortical signals to other cortical regions (Reichova and Sherman 2004, Llano and Sherman 2009, Theyel et al. 2010). In a theoretical neural network, a D-C circuit can prolong the initial depolarization from a direct monosynaptic signal, thereby amplifying and prolonging the total period of excitation. Although such means of extending the period of excitation on a neuron may be important for some forms of normal function, the same prolonged excitation from a D-C circuit may induce pathological states such as seizures.

Furthermore, focal or regional seizure activity that would not be intense enough to spread out to other limbic structures may be reinforced by the D-C excitatory amplification through the thalamus, thus permitting the recruitment of other structures. A simple model of how these D-C circuits may be constructed in the limbic network and how they may function in seizure activity is presented in Figure 8. That such circuitry may be key to seizure spread is not a new idea; thalamocortical circuits have been shown to be centrally involved in generalized seizures (Avoli and Gloor 1982, Meeren et al. 2002, 2009). However, the exact role they may play in the initiation and spread of limbic seizures has not been clear until now.

The anatomic connections that form the basis of the two D-C limbic circuits used in this study, and which give rise to the evoked potentials shown, are well known. Subiculum and CA1 efferents exit from its shallow layers (alveus), through the fimbria, fornix and lateral septum to the medial prefrontal, most strongly innervating the infralimbic and prelimbic regions. (Swanson 1981, Jay and Witter 1991, Degenaitus et al. 
2003) Subicular afferents also travel to the midline thalamus (Herkenham 1978, Namura et al. 1994 McKenna and Vertes, 2004, Cavdar et al. 2008). All midline thalamic nuclei, including the mediodorsal nucleus, innervate the medial PFC, with each midline thalamic nucleus overlapping its target fields in the PFC with the others. This thalamic afferents are excitatory (Van der Werf et al. 2002, Berendse and Groenewegen 1991, McFarland and Haber 2002, Hoover and Vertes 2007).

The shallow layers (II and III) of the piriform cortex project to the superficial layers of the medial and lateral entorhinal cortices, as well as to the mediodorsal nucleus (Cornwall and Phillipson 1988, Chapman and Racine 1997, Kerr et al. 2007). Several midline nuclei, including the paraventricular, parataneial and reuniens as well as the mediodorsal send excitatory projections to the both the medial and lateral EC (Van de Werf et al. 2002, Kerr et al. 2007). Stimulation of the mediodorsal has been shown to elicit excitatory potentials in the EC (Zhang and Bertram 2002). Both structures are considered susceptible to and participants in limbic seizure activity (McIntyre and Gilby, 2008).

There are other factors that must be considered in the interpretation of our results. First, while the TTX injections were placed directly into the midline thalamus, and the injection sites were verified visually, the proximity of the midline thalamus to a number of fiber tracts raises the possibility that the drug could have exerted its effect by blocking transmission of other pathways that pass near the injection site. Although this confounding factor cannot be completely excluded, the observation that injections outside of the mediodorsal region had no effect on the seizures or the evoked potentials make this explanation less likely.

The complex nature of the waveforms, as well as the tightly interconnected nature of the limbic network, suggests that multiple afferent pathways could contribute to the late evoked potential components in both pathways. Because of the potential contribution to the response from other sites that would likely appear around the same time as the thalamic component, we cannot say with any certainty that the late component is exclusively thalamic in origin. However, the observation that the late component changes with inactivation of the thalamus strongly suggests that a significant part of that 
component is from the thalaumus. Similarly, the peaks of the early component probably represent multiple local firing, including contributions from local interneurons. We suggest that the early component is initiated by a monosynaptic signal, and not that each peak represents an individual monosynaptic signal. No attempt was made to determine if signals represented depolarization or hyperpolarization.

This study suggests that limbic seizures initiate and spread through the enhanced excitation of divergent-convergent excitatory pathways, specifically those that pass through the midline thalamus. Although the monosynaptic path from the stimulus site to the target site induces a normal excitatory response, it is the additive contribution of the later-arriving multisynaptic thalamic excitatory drive, prolonging the monosynaptic excitation, that may be necessary to drive the network into a seizure. This possible role for the thalamus supports the theory that limbic epilepsies, in general, evolve in a hyperexcitable limbic network in which any structure within the network can act as the initiating site of a seizure (Bertram 1997, 2009, Bertram et al. 1998).

Although the focus of this study is the role of the midline thalamus in seizure activity, there are broader implications for this work in understanding the normal function of the thalamus in communication between cortical and subcortical structures. For example, Lopez et al. recently suggested that the role of the thalamus in hippocampalPFC communication may be important for remote spatial memory processing (Lopez et al. 2009), contributing to one of the many roles that the hippocampal-PFC pathway is thought to play (Laroche et al. 1990, Muldor et al. 1997, Degenetais et al. 2003, Kawashima et al. 2006). Furthermore, Van der Werf and colleagues (2003) suggested that the general role of the midline nuclei in declarative memory is to help focus on important information with regards to the contents and state of memory processes. The possible role of the thalamus in PC-EC communication has been studied less, although it may be posited that the thalamus assists with the communication of important primary olfactory information for application in memory processes (Kerr et al. 2007, Dickerson and Eichenbaum 2010). Our results suggest that, in studying the role of the thalamus in cortico-cortical or subcortical communication, the strong influence of divergentconvergent excitatory circuitry through the thalamus should not be ignored. 
In conclusion, the thalamus may serve to amplify excitatory input between limbic structures, and this ability may be an essential component of an enhanced excitatory drive necessary to drive limbic seizure activity.

Acknowledgements: This work was supported by NIH grant: NS25605. We thank John Williamson for his technical support. 


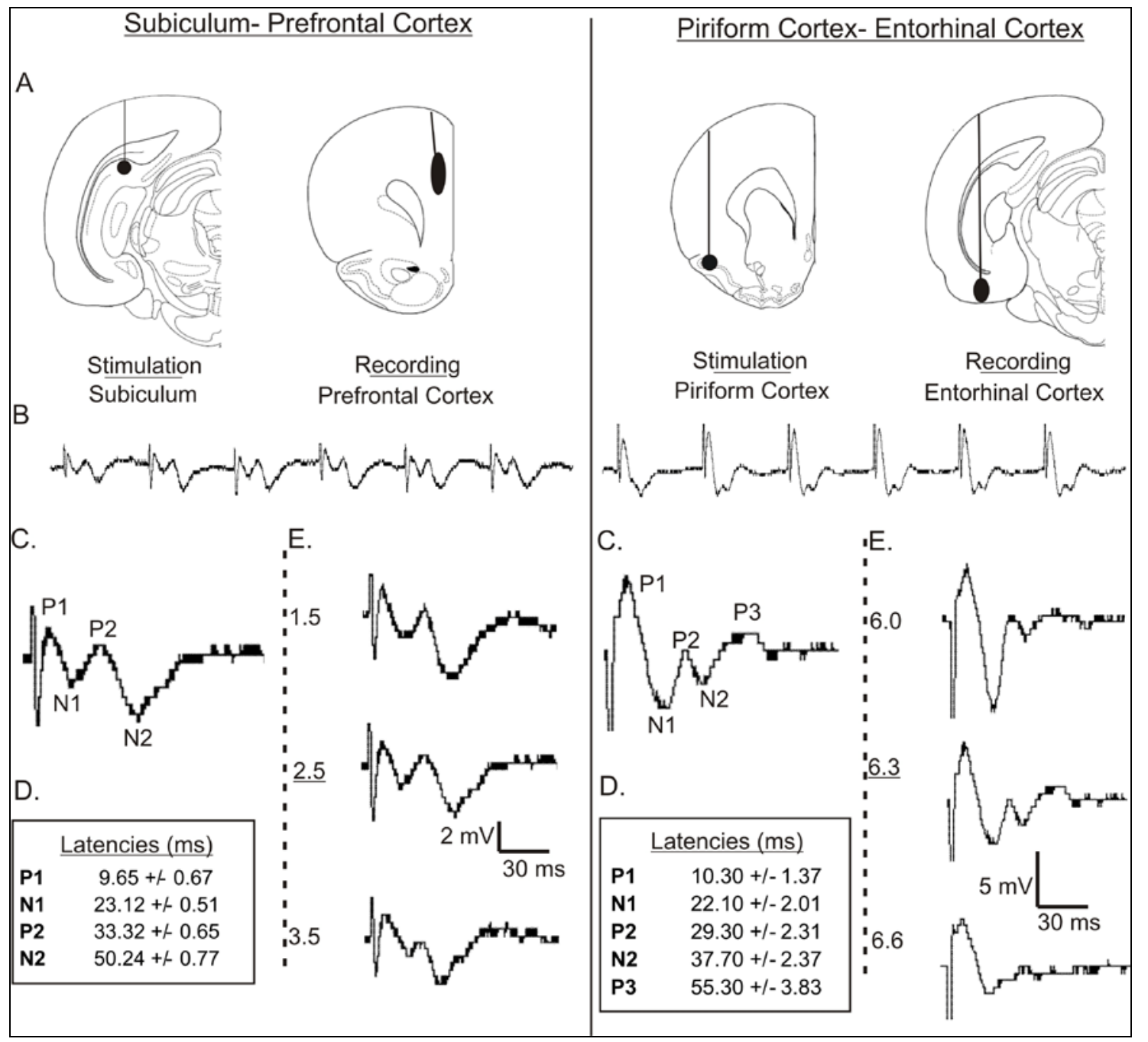

Figure 1: Evoked Potentials From Two Limbic Circuits

(A) Electrode placement for SB-PFC and PC-EC responses (Brain section illustrations from Paxinos and Watson 1996). (B) Typical $120 \mathrm{~ms}$ i.s.i. train from the stimulation-recording pairs that shows the increasing amplitudes of the responses, plateauing by the $4^{\text {th }}$ stimulation. (C) Typical $4^{\text {th }}$ response from each train, with major peaks labeled. (D) Average latencies for each peak of the $4^{\text {th }}$ responses. (E) Responses change with electrode depth. Position used in this study is underlined. 


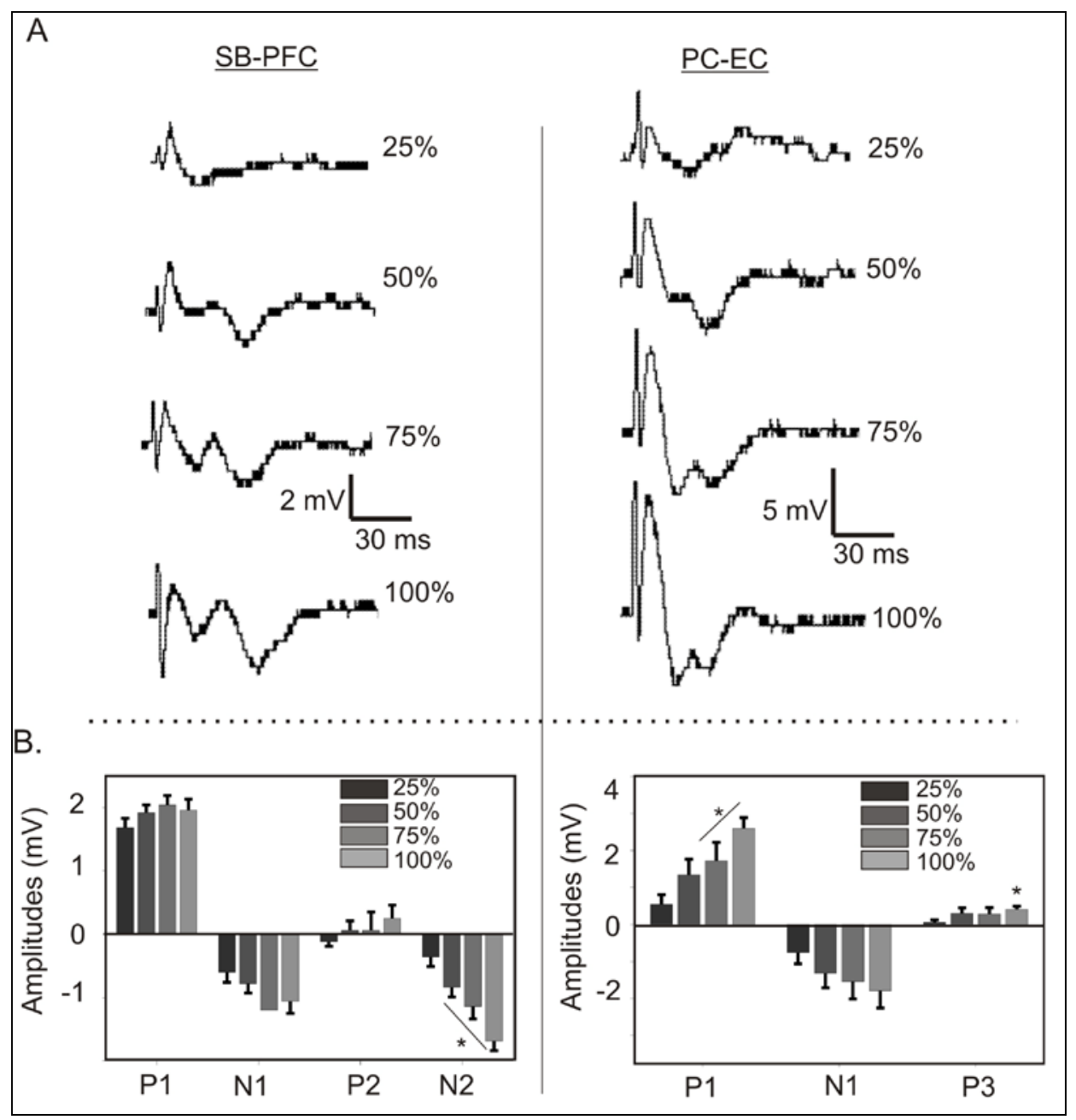

Figure 2: Effect of Increased Stimulation on Evoked Responses

To define maximal responses, as well as to determine the relative stimulus intensity needed to elicit each wave in the responses of both SB-PFC and PC-EC circuits, stimulus intensity was increased stepwise until maximum response. (A) Change of morphology and peak onset as intensities are increased, by increments of $25 \%$ of maximal stimulation. Note that responses at $50 \%$ intensity are different than those at $100 \%$, and that all peaks are fully formed at maximum intensity. (B) Average peak amplitudes as intensities are increased. ( ${ }^{*} \mathrm{P}<.05$ by student's t-test compared to $25 \%$ intensity). 


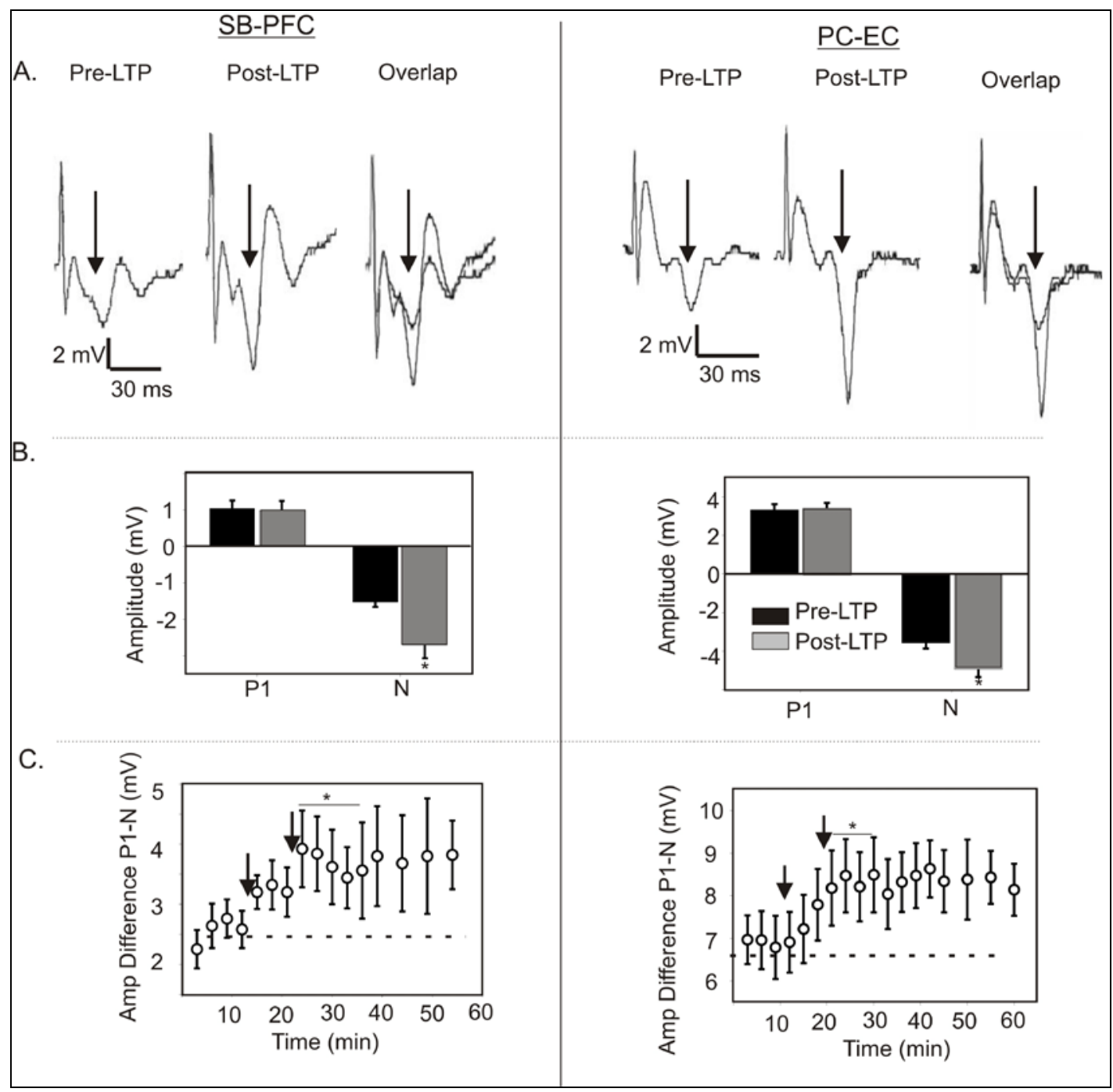

Figure 3: Long-Term Potentiation in Both Circuits Identifies Early Monosynaptic Component

(A) $2^{\text {nd }}$ responses from paired pulses in both pathways, taken at half-maximal intensity, before and after LTP was induced. Because of the low stimulation intensity, the peaks shown are not directly comparable to those in Figure 1 . An overlay of the waveforms shows that the first primary negative wave has increased in amplitude. (B) Amplitudes of the positive and negative peaks. In both cases, the negative peak increases significantly ( ${ }^{*} P<.05$ by student's t-test). (C) Changes in the amplitude of the negative peak over the course of the experiment. Note the rise in amplitude after the LTP stimuli were given. The latency of these peaks occurs earlier than the probable thalamic component of the response, and fall within the range of the monosynaptic component (see Figure 4). 


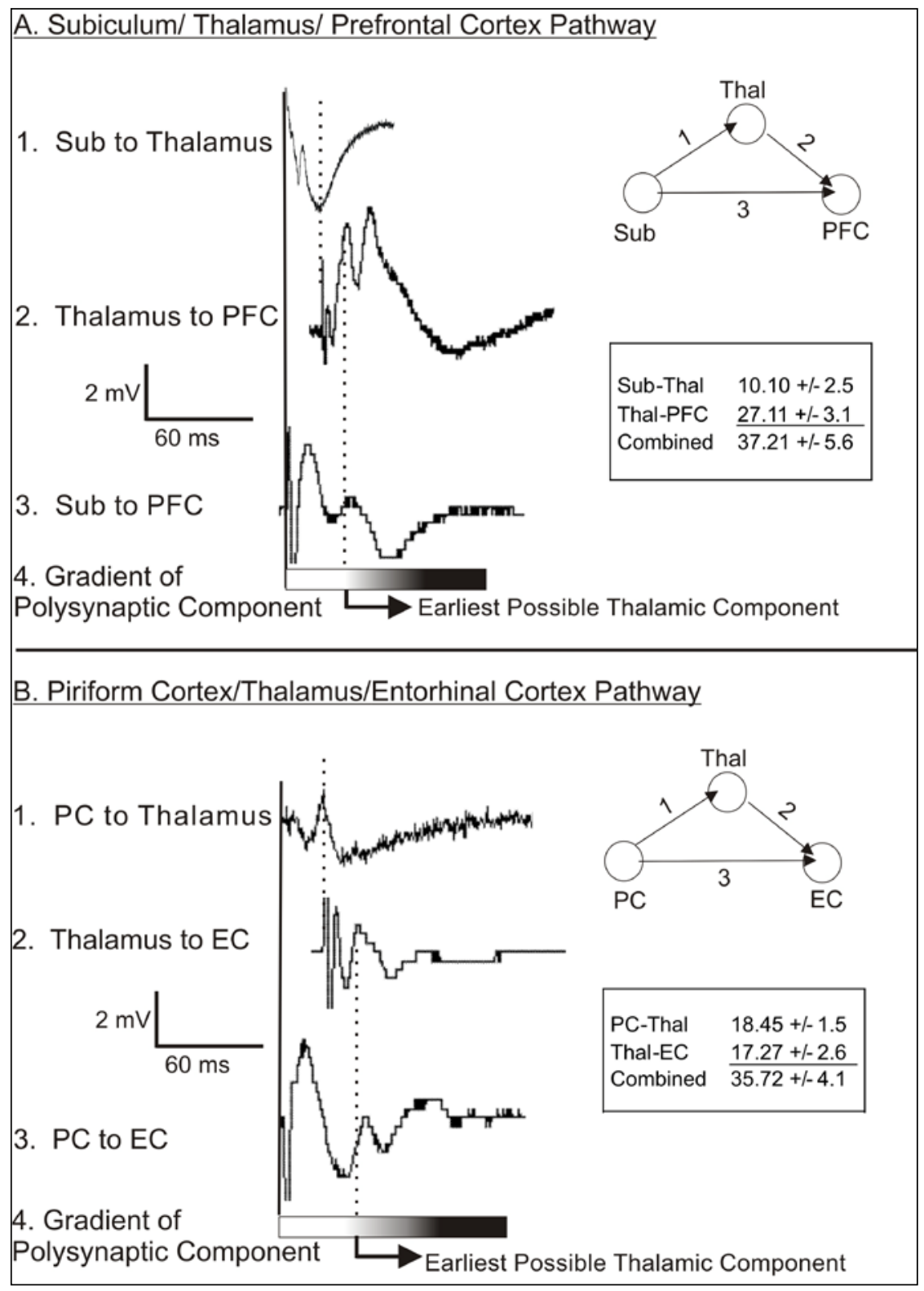

\section{Figure 4: Estimation of Onset of Thalamic Component}

To determine the approximate minimum time for the appearance for thalamic influence responses in the (A) SB-PFC and (B) PC-EC pathways, responses from the initiating site to the thalamus and the from the thalamus to the target site were obtained. Responses are aligned (1-3) and their latencies added to arrive at a minimum latency for thalamic involvement. The first major peak is treated as the monosynaptic response. The gradient bar (4) beneath the direct responses is placed to suggest that the onset of polysynaptic components occurs gradually within that suggested timeframe. The individual and combined latencies are quantified in the table inset. This figure is to be used and interpreted as a guide, and not an exact demonstration of thalamic influence in the direct response. 


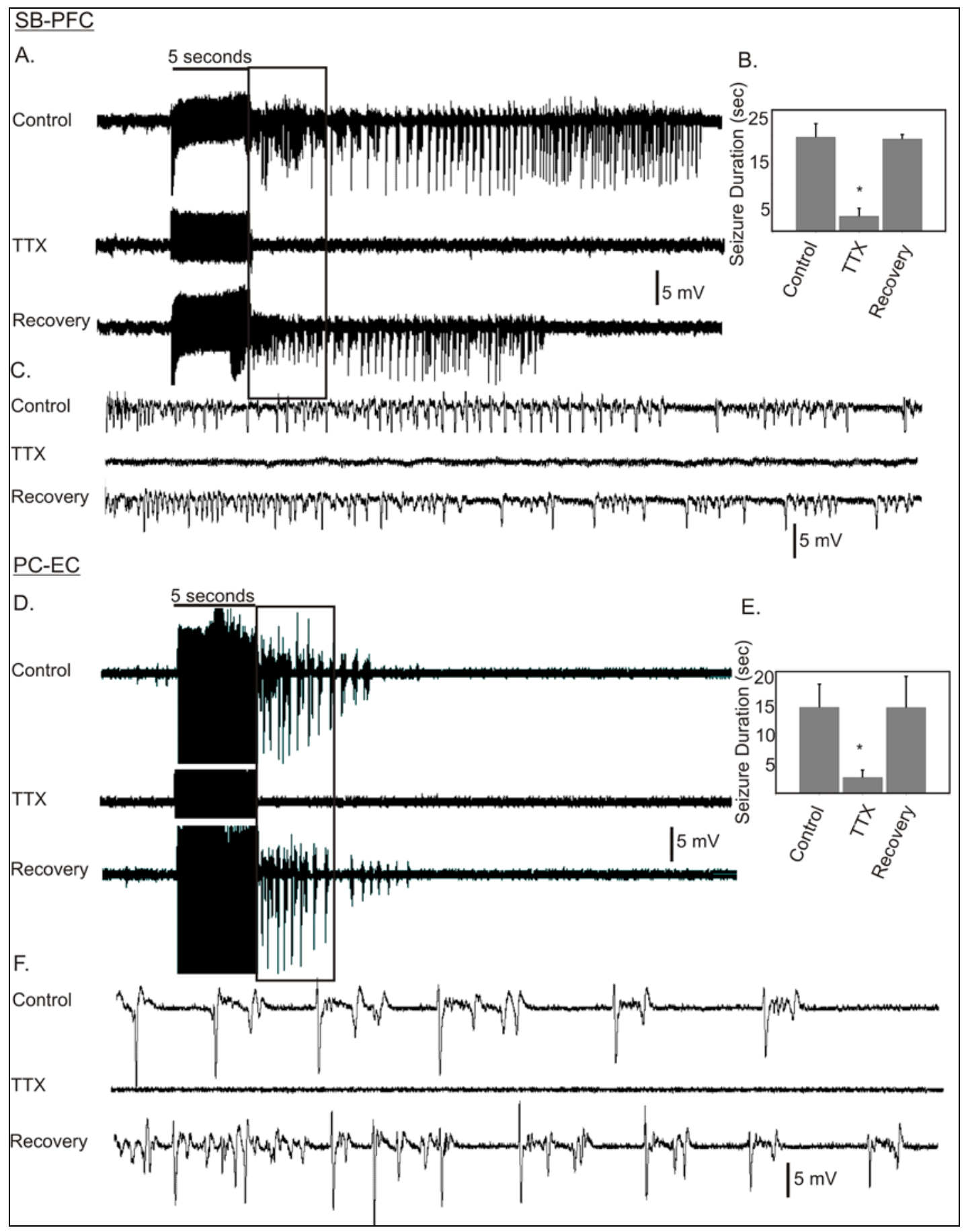

Figure 5: Limbic Seizures Affected by TTX in Midline Thalamus

(A) Limbic seizure afterdischarges before and after injection of TTX into the midline thalamus. Note that seizure activity is greatly reduced with TTX injection in both sets of responses. (B.) Average seizure durations before, during and after TTX with subsequent recovery. (C) Fivesecond samples from each trace (the boxed area on the full traces). 


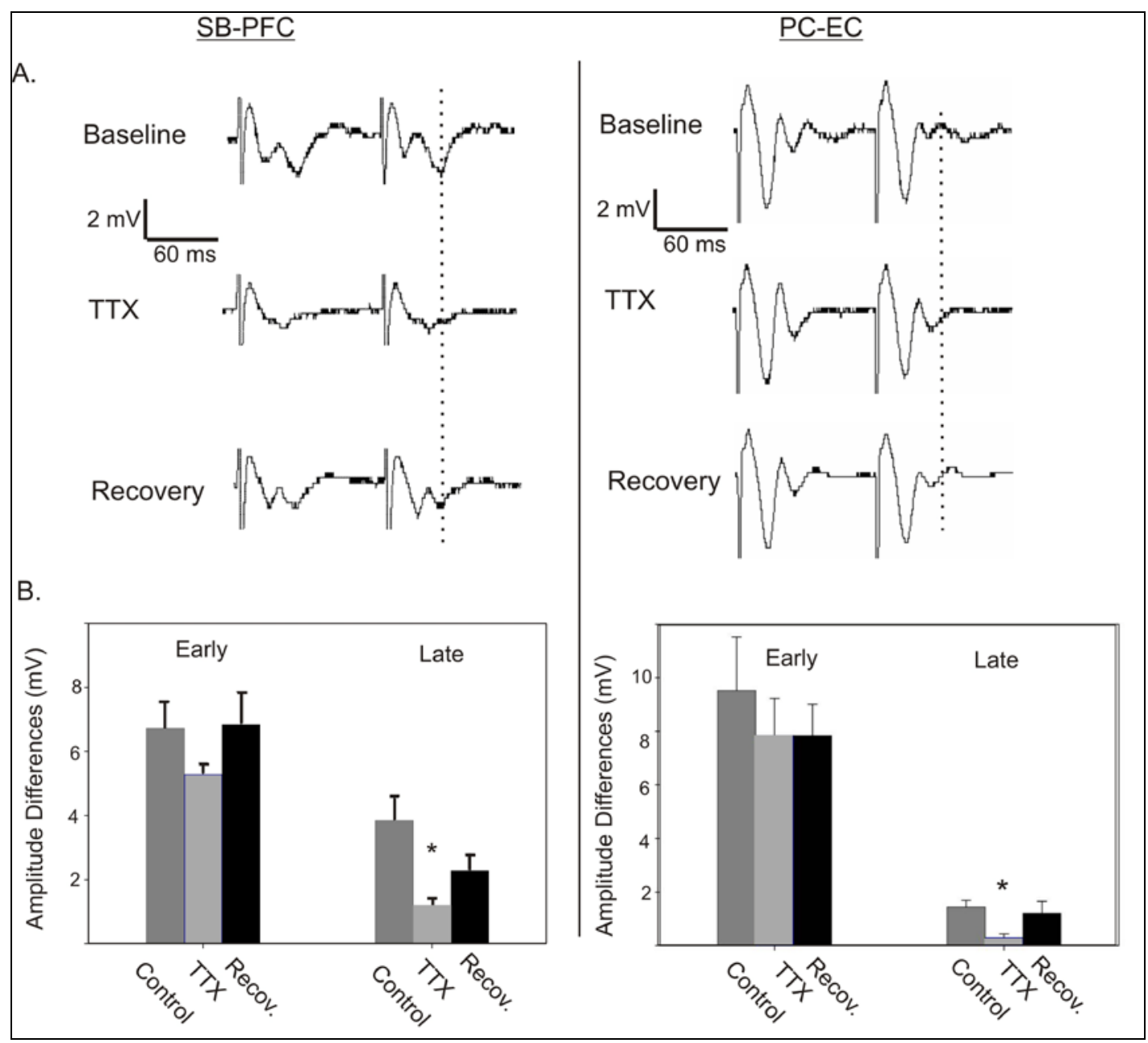

Figure 6: Evoked Potentials Affected by TTX in Midline Thalamus

(A) Sample averaged responses are shown for the SB-PFC $(n=6)$ and PC-EC $(n=5)$ circuits $\left(3^{\text {rd }}\right.$ and $4^{\text {th }}$ stimulations), before injection of TTX into the midline thalamus, after injection and after recovery. In both cases, the late component of the response is reduced in amplitude, and then recovers. (B) Amplitude differences between the peaks in the early (P1-N1) and late (P2-N2 in SB-PFC, P3-Baseline for PC-EC) components of the waveforms from the $4^{\text {th }}$ stimulation in both pathways, and how they change with TTX injection in the thalamus. In both cases, the changes in the late components of the response are significantly reduced. ${ }^{*} \mathrm{P}<.05$ by paired t-test. 


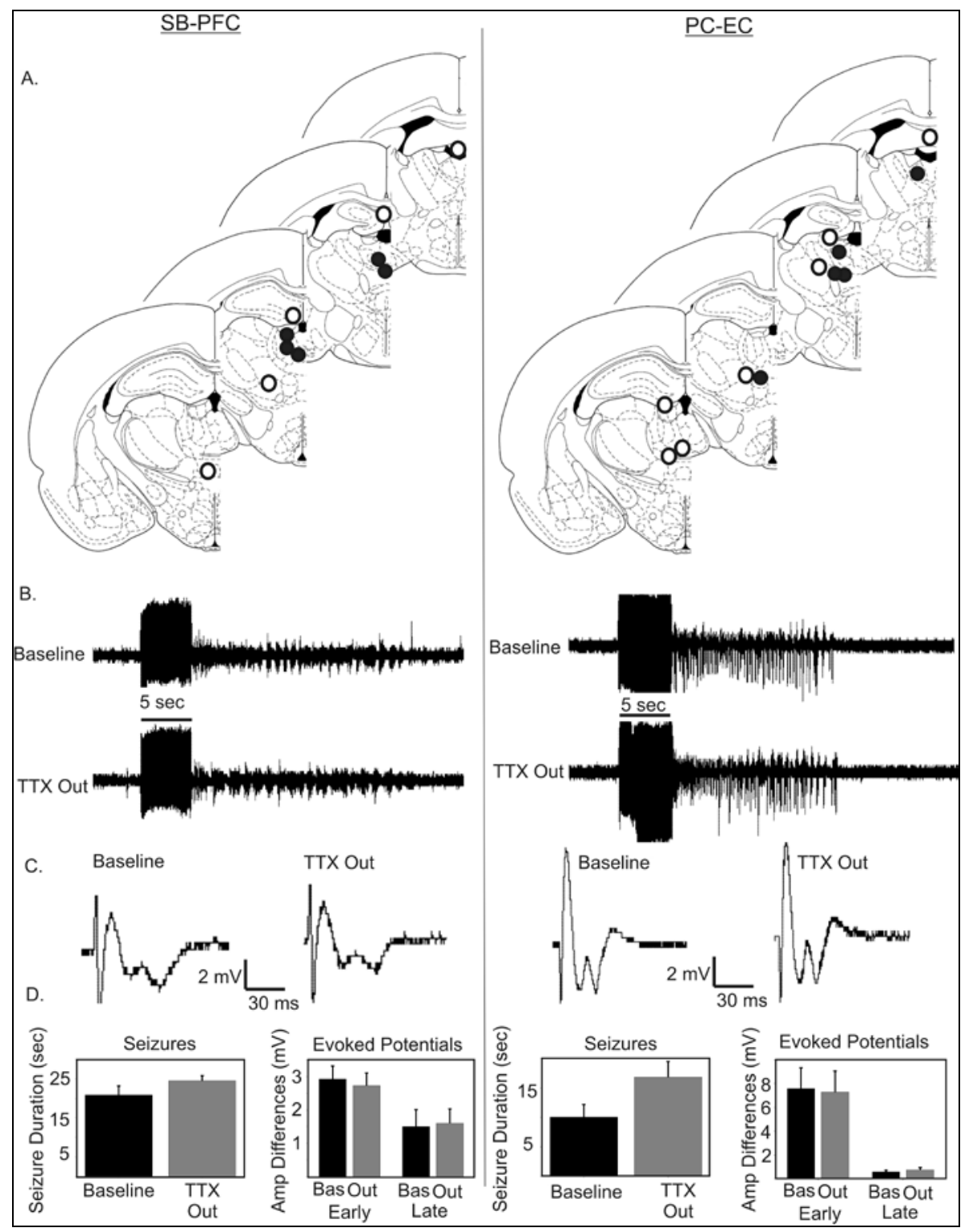

Figure 7: Position Specificity of Effect of TTX on Seizures and Evoked Potentials

To show that the effects shown are specific to TTX injections in the mediodorsal nucleus, injections within and without that region are compared. (A) Both effective (filled circles) and ineffective (open circles) injections of TTX into the area of the midline thalamus are shown for both SB-PFC and PC-EC pathways. (Brain section illustrations from Paxinos and Watson 1996). (B) Sample seizure from an ineffective injection outside of the mediodorsal nucleus, showing a seizure before injection, and 30 minutes post injection. No change is duration is seen. (C) Evoked responses corresponding to the seizures shown, with no change seen. (D) Summary of average seizure durations and evoked potential peak amplitudes in both sets of animals with injections outside the MD ( $n=5$ for SB-PFC, $n=6$ for PC-EC). 


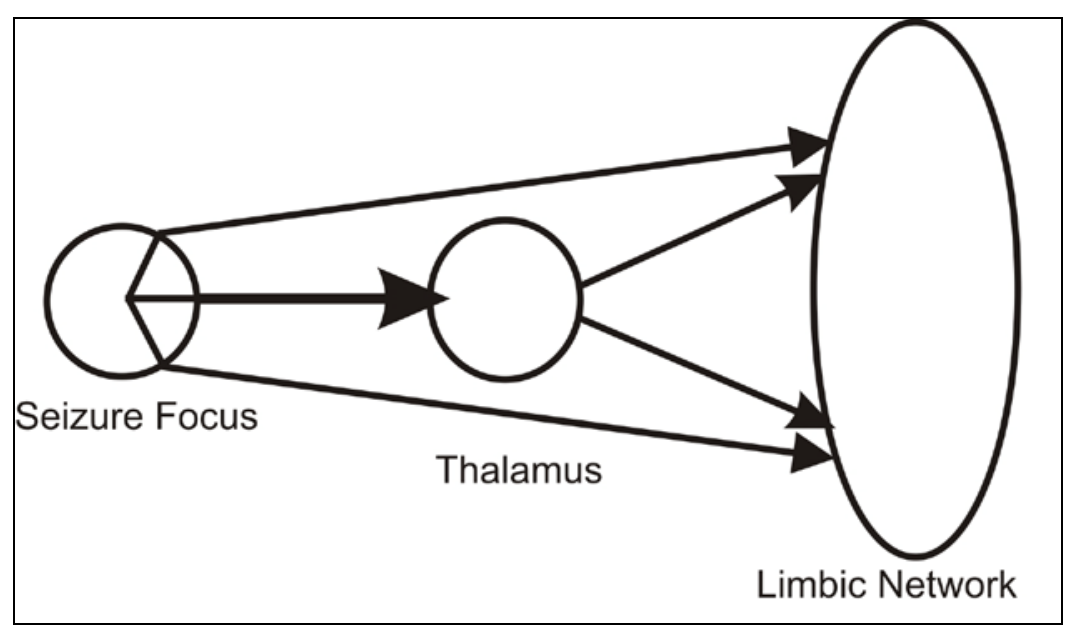

Figure 8: Representation of Divergent-Convergent Excitatory Circuits in the Limbic System

Model of a proposed role for the thalamus in limbic seizure circuits. A seizure is initiated in a region in the network (focus). Excitatory activity affects signaling to all the monosynaptic afferent targets of the region, including the midline thalamus. The thalamus, in turn, sends an additional, excitatory signal to all of the monosynaptic targets of the focus. This additional excitation assists in recruiting and driving the target structures into seizure activity by prolonged excitation. 


\section{Chapter IV}

\section{Increased GABA-ergic Inhibition in the Midline Thalamus Affects Signaling and Seizure Spread in the Hippocampus-Prefrontal Cortex Pathway}

Summary

Introduction: The midline thalamus is an important component of the circuitry in limbic seizures, but it is unclear how synaptic modulation of the thalamus affects that circuitry. In this study, we wished to understand how synaptic modulation of the thalamus can affect inter-regional signaling and seizure spread in the limbic network.

Methods: We examined the effect of GABA modulation of the mediodorsal (MD) region of the thalamus on responses in the prefrontal cortex (PFC) by stimulation of the subiculum (SB). Muscimol, a GABA-A agonist, was injected into the MD, and the effect on local responses to subiculum stimulation were examined. Evoked potentials were generated in the PFC by low frequency stimulation of the SB, and seizures were generated in the subiculum by repeated $20 \mathrm{~Hz}$ stimulations. The effect of muscimol in the MD on the evoked potentials and seizures was measured.

Results: Thalamic responses to stimulation of the subiculum were reduced in the presence of muscimol. Reduction of the amplitudes of evoked potentials in the MD resulted in an attenuation of the late, thalamic components of the responses in the PFC, as well as of seizure durations.

Conclusion: Modulation of inhibitory synapses in the midline thalamus not only causes changes within the thalamus, but has broader effects on the limbic network. This work provides further evidence that synaptic modulation within the midline thalamus alters system excitability more broadly and reduces seizure activity. 


\section{Introduction}

Mesial temporal lobe epilepsy (mTLE) is a common neurological disorder characterized by seizures arising in the limbic network. At present, about 30\% of TLE patients don't respond to medications and require surgery to control their seizures (Kwan and Sperling 2009). For more effective therapies to be developed, a better understanding of the underlying circuitry of seizure activity in the limbic system is needed.

The midline thalamus is a potential candidate for direct clinical intervention in mTLE, because it is connected to most limbic structures involved in mTLE (Ray et al. 1992, Van der Werf et al. 2002, Berendse and Groenewegen 1991, Hoover and Vertes 2007), and work to date has shown that it is a critical component in the initiation and spread of seizure in animal models of mTLE. For example, injection of GABAergic drugs into the thalamus alters seizure activity in animal models (Patel et al. 1988, Miller and Ferendelli 1990, Cassidy and Gale 1998, Bertram et al. 2001, 2008). It has long been assumed that the reason that targeting the thalamus with these drugs is so effective is because it causes a two step reaction: a local change in neuronal circuitry, which, in turn, leads to broader effects to its afferent connections. However, the details of why this intervention suppresses seizures have been unclear.

To resolve this question, we sought to identify both the local and widespread effects of GABAergic modulation of the midline thalamus. We hypothesized that modifying synaptic responses in the MD would reduce its excitatory effect on components of the seizure circuit.

We pursued this hypothesis by stimulating the subiculum, (the output region of the hippocampus), and recording in both the thalamus and the prefrontal cortex as the GABA-A agonist muscimol was injected into the MD. The subiculum-prefrontal cortex (SB-PFC) pathway was chosen for these experiments for several reasons. First, both the hippocampus and PFC have been shown to participate in both chronic and kindled models of TLE (Sloan and Bertram 2009). Second, there is a known monosynaptic pathway between the subiculum and the PFC, as well as a potential polysynaptic path that 
connects the two regions through the midline thalamus. This forms a potential divergent-convergent circuit that has been shown to be involved in limbic seizure activity.

The goal of this study was to evaluate whether there were measurable circuit effects after modulation of the mediodorsal nucleus by the GABA agonist muscimol.

\section{Materials and Methods}

All animals were used in accordance with protocols approved by the Animal Care and Use Committee (ACUC) at the University of Virginia. Naïve adult male SpragueDawley rats (Hilltop Laboratories, 250-350 g) were used for all experiments.

\section{SB-Thalamus Pathway}

Electrode and Cannula Placement in SB-Thalamus Pathway

We first sought to show the effect of a GABA agonist on synaptic responses in the thalamus. Animals were put under urethane anesthesia (1.2 g/kg, i.p.) and placed in a multi-arm stereotactic frame. Body temperature was maintained at $37^{\circ} \mathrm{C}$ by a water blanket controlled with a rectal thermistor. The stimulating and recording electrodes and the cannula for drug perfusion in this study were inserted stereotactically using target coordinates derived from the rat brain atlas of Paxinos and Watson (1998). One bipolar, stainless steel stimulating electrode was placed into the ipsilateral subiculum (-6.3 to -7.0 AP, 4.0-5.5 ML, 2.5 to $8.0 \mathrm{~mm} \mathrm{DV}$ ). To find optimal responses, a glass micro-pipette recording electrode ( $1 \mathrm{M} \Omega$ tip resistance) was filled with $0.9 \% \mathrm{NaCl}$ and $1 \%$ Fast Green dye and placed in the midline thalamus (from Bregma, in mm: -1.8 to -3.2 AP, 0.4 to 0.8 ML, -4.5 to -5.3 DV below dura). The depths of the stimulating and recording electrodes were adjusted stepwise to achieve a consistent and maximal response (see Figure 1).

For local drug injection into the thalamus, a custom holder was used to hold the glass recording electrode and a stainless steel perfusion cannula (25G) close together in the same sagittal plane. Both the electrode and cannula tips were placed 5.0-5.8 mm 
below the dura so that the tips were less than $1 \mathrm{~mm}$ apart. The recording electrode was vertical to the brain surface, while the cannula tip was inserted at an angle (5-12 $)$ toward the recording electrode so.

\section{Recording and Drug Delivery in SB-Thalamic Pathway}

Evoked potentials in the thalamus were induced by single (0.1-0.2 ms duration monophasic square wave) stimulation of the subiculum. Five responses, from stimulations delivered every 10 seconds, were averaged at each stage of the experiment and used for analysis.

We first obtained a stable maximal amplitude response, and began the infusion of vehicle (ACSF: 127 mM NaCL, 2 mM KCl, 1.5 mM CaCl2, 1.5 mM MgSO4, 25.7 mM NaHCO3, $1.1 \mathrm{mM}$ KH2PO4 and $10 \mathrm{mM}$ Dextrose) for at least $30 \mathrm{~min}$. at 0.1-0.2 $\mu \mathrm{l} / \mathrm{min}$. Muscimol (dissolved into ACSF) was infused through the same cannula by changing syringes using a fluid switch. $0.5 \mathrm{mM}$ muscimol was infused for 30 minutes near the tip of the recording electrode, after which ACSF flow was resumed. Recovery was measured after a minimum of 30 min of wash.

At the end of each experiment, the electrode position was marked for histological confirmation by iontophoresing Fast Green dye from the recording electrode into the surrounding tissue by using a DC current (20-50 $\mu \mathrm{A}$, 5-15 min). Positive DC current (10V for 5-10 sec) was passed through the negative tip of the stimulating electrode to deposit iron from the electrode into the surrounding tissue. The positive DC current was also passed through the drug infusion cannula to confirm the cannula position. Then, the animals were decapitated while still under anesthesia, and the brains were removed and placed into a fixative consisting of $1 \%$ potassium ferrocyanide and $4.0 \%$ paraformaldehyde. Brains were sectioned to confirm electrode placement. For injection studies, only experiments with the electrodes in the designated areas were included in the analysis.

Measurements of the evoked responses include the peak amplitude of the population spike and post-synaptic potential (PSP) from the baseline and the latency of 
the population spike peak. Responses were compared using student t-test and paired ttest. Significance was set at the $\mathrm{p}<.05$ level.

\section{SB-PFC Pathway}

\section{Evoked Potentials}

In order to determine whether synaptic modulation of the MD caused a change in a limbic pathway, we evoked field responses in the PFC by SB stimulation. Animal preparation for these experiments was as described above. The stimulating electrode was placed in the dorsal SB (From bregma, in mm: -6.3 to -7.0 AP, 4.0 to $-4.5 \mathrm{ML},-2.7$ to 3.5 DV), corresponding to the site used in the SB-Thalamus pathway experiments. The glass recording electrode was placed in the ipsilateral medial PFC (2.8 to 3.2 AP, $0.5 \mathrm{ML}$, -1.5 to $-3.5 \mathrm{DV})$. This recording site was chosen based on previous work that demonstrated strong afferent connections from both the SB and midline thalamus to the PFC, so that there was a likely combination of mono- and polysynaptic components in the response to SB stimulation.

Electrode positions were adjusted to find maximal response amplitudes. Field potentials were obtained through an Axon Smart Probe and a Cyberamp 380. Signals were digitized through a Digidata 1440A-and analyzed using Axoscope 8.0 software (Molecular Devices, CA, USA).

Field potentials were elicited by a $120 \mathrm{~ms}$ inter-stim interval (i.s.i.) ( 8.3 Hz) train of stimulations. This i.s.i. was chosen in order to maximize the likelihood of involvement of the thalamus in the field potential. Direct stimulation of the thalamus at this frequency elicits a thalamus-specific "recruiting response” in which field potential peaks become increasingly augmented (Sloan and Bertram 2009, Dempsey and Morison 1942, Verzeano et al. 1953, Bazhenov et al. 1998). To ensure that all peaks were fully formed, and because response amplitudes increased over the repeated stimulations of the train until plateauing, the $4^{\text {th }}$ stimulation of the train was used for all analyses. Amplitudes for each peak were measured from the baseline value occurring immediately prior to the stimulation. 
Waveforms generated by this stimulation protocol consisted of multiple peaks: two positive (P1 and P2) and two negative (N1 and N2) peaks. The composition of these evoked field responses is complex, and likely originates from multiple circuits. Because determining a potential thalamic component of these EP's in necessary, we wished to identify a minimal latency in which a polysynaptic response throughout the thalamus would appear in the PFC. We added field response latencies derived from stimulusrecording experiments in the SB-MD pathway from this paper $(10.10+/-2.5 \mathrm{~ms})$, and the MD-PFC pathway from previous work (27.11 +/- $3.1 \mathrm{~ms}$ ) (Sloan and Bertram 2009). These latencies suggest that a thalamic influence would appear in a PFC field response to subiculum stimulation after approximately $35 \mathrm{~ms}$. Therefore, for the purposes of this study, peaks occurring prior to $35 \mathrm{~ms}$ were considered to contain the monosynaptic signal, and those occurring after 35 ms were considered to contain a thalamic influence, among other possible polysynaptic influences (Fig 3C).

Stimulations were given at a maximal amplitude of 70V (maximal current 2.3-4.7 $\mathrm{mA}$, at a typical stimulating electrode resistance of $15-30 \mathrm{k}$ ). The stimulus intensity was increased until a maximum response was gained, or until 70V. A minimum of five trains, each lasting 1 second, were averaged with each acquisition. Recordings were acquired every ten minutes.

\section{Seizures}

In the course of this experiment, there was a standard relationship between the evoked potential and the induced seizures. For each evoked potential train that was recorded, seizure afterdischarges were elicited immediately afterward by a $20 \mathrm{~Hz}$ train of monophasic pulses of $0.17 \mathrm{~ms}$ duration for five seconds at the same intensity that was used for the evoked potentials. Seizures were identified as large, repetitive spike bursts beginning immediately following the end of the stimulus train. Seizure termination was determined by the end of the last spike burst. For each experiment, a minimum of ten stimulus trains were required to induce seizures of a consistent duration. Once a stable seizure duration was achieved, defined as a minimum of three consecutive seizures with 
durations longer than 10 seconds with less than 30\% variation between them, drug was injected.

For analysis, three evoked potential/ seizure pairs were used for each rat. The first was the recording made just prior to injection of drug (baseline value). The second was the recording with the maximal reduction in seizure duration occurring at least 10 minutes after injection, (to allow for adequate drug diffusion). The third was the recording taken a minimum of 20 min after the seizure had returned to a baseline duration.

\section{Muscimol Suppression of Seizures}

Muscimol was injected into the midline thalamus using a Hamilton $25 \mu 1$ microsyringe with a flat tip. The tip was placed into the ipsilateral mediodorsal nucleus (-1.8 to -2.8 AP, 0.5 ML, -5.0 to -6.0 DV). All drug injections were performed after a baseline of evoked potentials and kindled afterdischarges were achieved. $5.0 \mathrm{mM}$ muscimol, with a standard injection volume of $0.75 \mu$ l over five minutes. The concentration used here was higher than that used in the local SB-Thal experiments to ensure that more of the thalamus was exposed to an effective concentration of the drug. Injections were made slowly over the course of three minutes. A successful injection was one in which seizure durations were suppressed by more than 50\%.

At the end of the experiment, electrode placements were determined as described above and compared to the results of the injection. Injections outside of the specified region of the MD were ineffective, and were grouped separately from effective injections within the region.

\section{Results}

\section{Effect of Local Muscimol in Midline Thalamus on SB-Thal Responses}

Evoked field responses to SB stimulation in the midline thalamus were recorded. Both stimulation and recording electrodes were adjusted within the structures. The most consistent maximal responses were acquired when the stimulating electrode was in the 
dorsal region of the subiculum (between -2.5 to -3.5 mm below dura), and when the recording electrode was in the central to dorsal MD (Figure 1). Standard evoked potentials with this pairing consisted of very brief positive and negative spikes, followed by a much larger and longer negative post-synaptic potential of an average latency of $10.10+/ 2.5 \mathrm{~ms}$.

We injected muscimol and recorded the change in the evoked potentials. The muscimol injections caused a marked reduction of the post-synaptic potential which recovered as the drug washed out (Figure 2).

\section{Effect of GABAergic Modulation of Thalamus on SB-PFC Seizures}

In order to ensure that the drug delivery to the MD was both on target and sufficient to stop seizures, we induced seizures in the subiculum (the dorsal region used in the previous experiment) and recorded them in the PFC, at an average electrode depth of $2.5 \mathrm{~mm}$ (Figure 3A). Seizures were induced by applying regular $20 \mathrm{~Hz}$ stimulations at 10 minute intervals. These stimulations caused seizures of an average duration of 23.48 $+/-2.12$ sec. It has been shown in multiple studies that the effect of drug injections on seizures is largely site-specific (Bertram et al. 2008, Patel et al. 1988, Miller and Ferendelli 1990, Cassidy and Gale 1998). Therefore, we could judge the accuracy and effectiveness of an injection by the effect that it had on seizure duration. We confirmed the accuracy of these injections histologically.

Successful injections of muscimol caused a reduction of seizure durations (Figure 2) which lasted for 30-50 minutes before recovering to baseline duration. Successful modulation of seizure duration was considered an indication of an injection within the midline thalamus. Injections outside the region did not induce changes in seizure duration or evoked potentials, but were used as controls for site specificity (Figure 2A).

\section{Effect of GABAergic Modulation of Thalamus on SB-PFC Evoked Potentials}

To determine the network effects of increased inhibition of the MD, we examined the effect of muscimol injection into the MD on evoked potentials in the SB-PFC pathway (Figure 3). It has been shown previously that stimulation of the subiculum in 
trains between 7-9 $\mathrm{Hz}$ at maximal stimulation intensity yields a complex waveform that is reproducible. This frequency was chosen to maximize the likelihood of thalamic involvement in the response, as stimulation of the thalamus at that frequency is known to induce a progressive response amplification, or "recruiting response” (Sloan and Bertram 2009, Dempsey and Morison 1942, Verzeano et al. 1953, Bazhenov et al. 1998).

The waveforms from these trains can be approximately divided into early and late components; the early components primarily contain the monosynaptic signal, while the late components contain any additional polysynaptic influences. We used the $4^{\text {th }}$ response of the stimulus train because the waveforms are completely formed and at their largest amplitudes by that point in the train. The waveforms consist of two positive (P1 and P2) and two negative (N1 and N2) peaks. We grouped these peaks as "early” (P1 and N1, before $35 \mathrm{~ms}$ ) and "late” (P2 and N2, after $35 \mathrm{~ms}$ ), and considered the late component to be the more likely to contain a thalamic influence than the early component, as described in the methods (Fig 3C).

Injection of muscimol into the mediodorsal nucleus also caused a reduction in late component of the response, although to a lesser degree and for a longer period of time. The late component, especially the N2 peak, was preferentially affected (Figure 3 D and E). Again, injections that were outside the midline thalamus, and that did not affect seizure durations, did not affect the evoked potentials (Figure 3F). These results demonstrate that local modulation of the thalamus has a widespread, network-level effect.

\section{Discussion}

This study provides a network-level mechanism for the observation that application of drugs acting on GABA-A receptors in the midline thalamic region modulate limbic seizures. The drugs act by increasing synaptic inhibition, reducing the responsiveness of the thalamus, which subsequently causes the thalamic excitatory drive on other target regions to be reduced. This excitatory drive may be a significant component in the initiation and spread of limbic seizures. 
The results of this study, in conjunction with a number of other studies, suggest that the midline thalamic nuclei may be a key component of a divergent-convergent circuit in which at least two pathways connect one region to another: a direct monosynaptic connection and an indirect, polysynaptic connection that passes through the thalamus (Figure 5). This divergent-convergent circuitry has the capacity to amplify excitation at the target region by prolonging depolarization. Similar circuits have been shown in non-limbic thalamocortical circuits (Reichova and Sherman 2004, Llano and Sherman 2009, Theyel et al. 2010). The convergence of afferent hippocampal and thalamic signals in the PFC has been shown in the PFC (Floresco and Grace 2003). In seizures, this circuitry may facilitate the spread of seizure activity between two regions.

To our knowledge, this is the first study demonstrating that GABAergic modulation of the midline thalamus has a network-level effect that explains the seizure modulation observed in previous experiments. The thalamus has long been known to participate in generalized seizures (Pellegrini et al. 1978), but explorations into its physiological role in limbic seizures required animal models, as well as a better knowledge of the potential circuit components, that were not available until relatively recently. Injection of muscimol and other GABA-ergic drugs into the thalamus to alter seizure activity in various animal models was a common, and often successful, experiment between the years 1987-1995 (Patel et al. 1988, Lee et al. 1989, Miller and Ferrendelli 1990, Garant et al. 1993). The work of Cassidy and Gale furthered this work and showed that a site-specific effect of midline thalamic muscimol injection in a model of limbic seizures, in which the seizures were directly initiated in the piriform cortex (Cassidy and Gale 1998). Similar work was also performed recently in a model of awake, kindled rats with seizures generated in the hippocampus (Bertram et al. 2008).

Until now, no work had yet come forward to demonstrate why these experiments worked, although it was posited by Miller and Ferrendelli that the thalamus "acts to modify the excitability of other structures more directly involved in the process [of seizure origination and spread]” (1990), and despite the mounting evidence that the thalamus is a critical component of limbic seizure circuitry. The present study provides a mechanism for the success of those experiments. Additionally, this study provides a 
rationale for findings that structures that send GABAergic projections into the midline thalamus, such as the reticular nucleus (Cavdar 2008, Nanobashvili et al. 2003) and the ventral forebrain (Churchill 1996, Daransart et al. 1999), have the ability to modulate seizures. This study suggests that synaptic modulation in the thalamus may have clinical applications.

Although much work is yet to be done to prove that the midline thalamus is a useful clinical target in human TLE patients, evidence from both animal models and clinical studies has been encouraging. The corroborating evidence that the midline thalamus plays a role in mTLE seizures in humans is building (Blumenfeld 2004, Bonilha 2005, Guye et al 2006, Mueller 2009). Investigations involving deep brain stimulation of the thalamus have already begun in human patients (Fisher et al. 2010). This work adds evidence that direct pharmacological interventions may also be feasible. However, we stress that this study, along with many previous studies, demonstrates that any injections or interventions must be in precisely the correct location in the thalamus, and that interventions that are outside of the critical midline thalamic region- even if they are close- may be ineffective (Cassidy and Gale 1998, Bertram et al. 2008, Sloan et al 2010submitted). Site-specificity is a critical consideration for any future clinical therapies involving the midline thalamus.

There are potential limitations in this study. Field potentials, by definition, include responses from many neurons, making it difficult to distinguish between excitatory and inhibitory activity. The exact effects of thalamic modulation on individual cells or sub-populations in the PFC were not resolved. The diversity of cells that receive both hippocampal and thalamic inputs has been shown (Floresco and Grace 2003). For the purposes of this study, an examination of SB-PFC connectivity was sufficient to demonstrate the influence and importance of the midline thalamus on its signaling. In conclusion, modulation of thalamic inhibition has effects outside of the thalamic region; it has the potential to indirectly reduce normal signaling between cortical regions of the limbic network. This physiological effect should be taken into consideration in studies of basic seizure circuitry. 


\section{Acknowledgements}

This work was supported by NIH grant: NS25605. We thank John Williamson for his technical support. None of the authors has any conflict of interest to disclose.

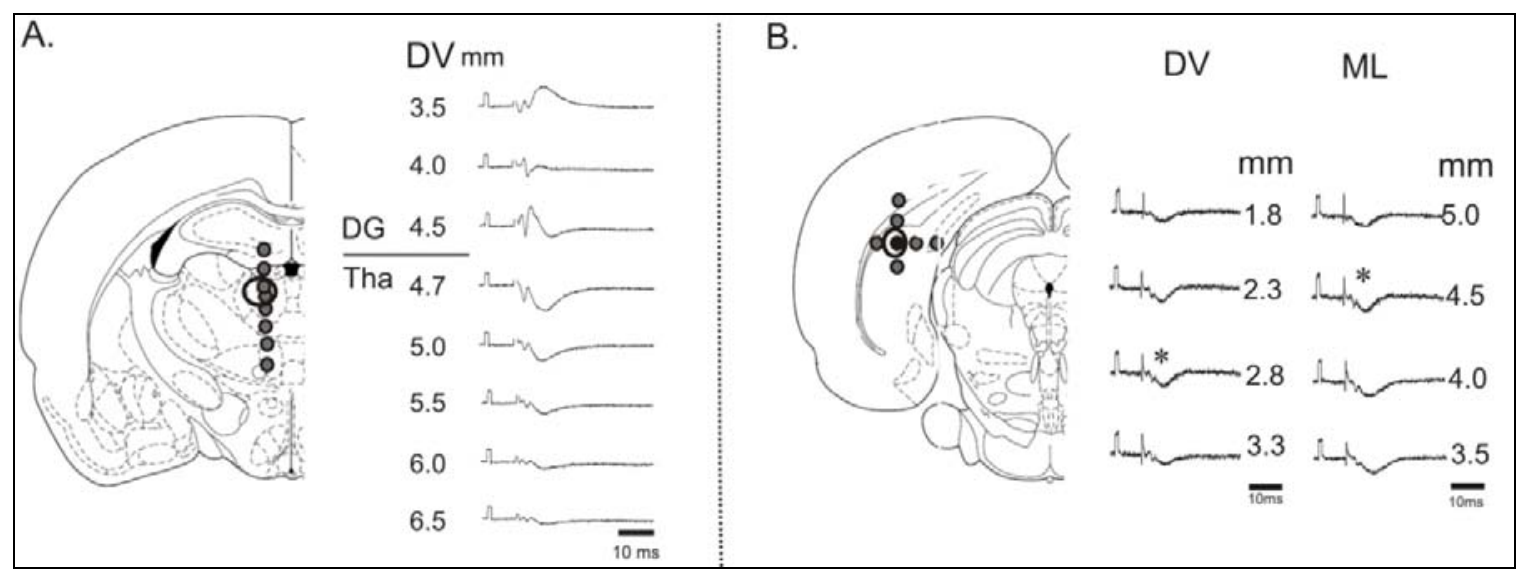

Figure 1: Stimulation of Subiculum Induces Field Responses in Mediodorsal Thalamus To find optimal responses, pairs of electrode placement depths were compared. (A) The optimal thalamic recording site was found in the mediodorsal nucleus ( $\sim 5 \mathrm{~mm}$ below dura) (B) The optimal stimulation site in the subiculum was found in the dorsal region ( $\sim 2.8$ below dura). The optimal site was found in the dorsal-most region of the subiculum.

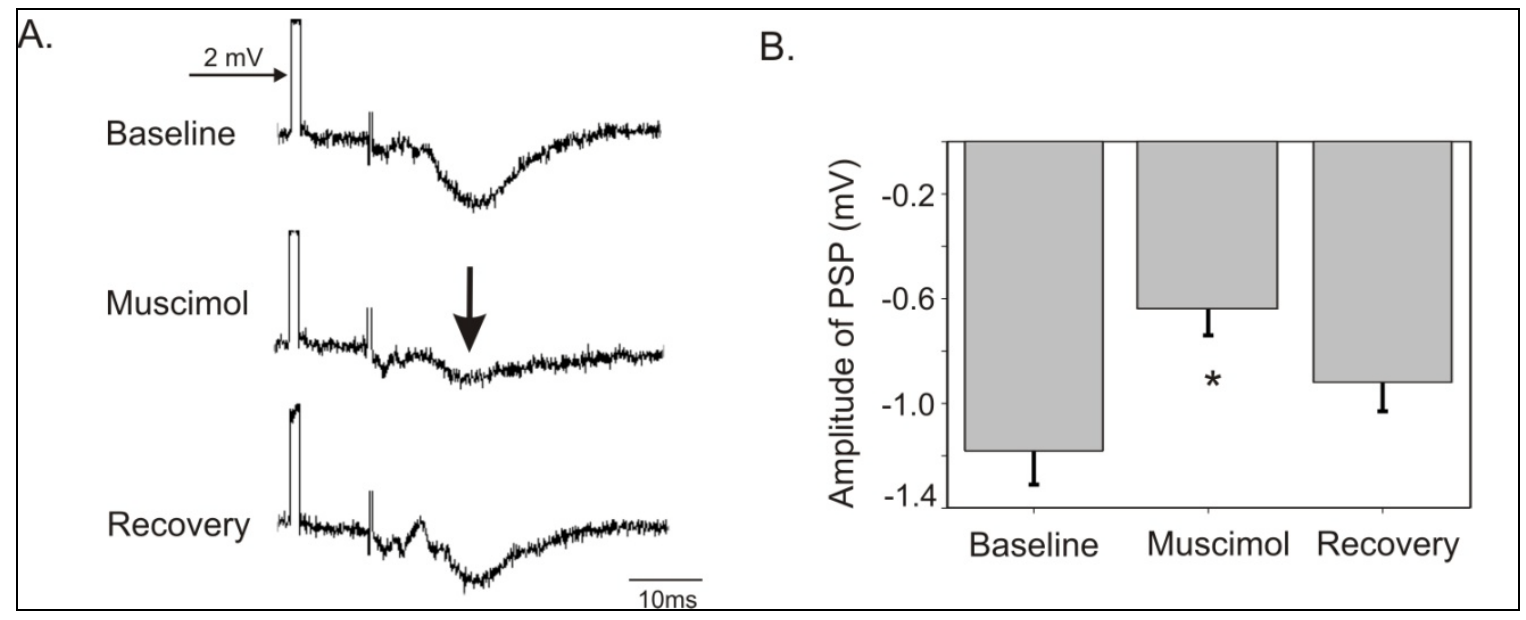

Figure 2: Responses in the Midline Thalamus to Subiculum Stimulation Reduced With Local Injection of Muscimol

(A) Injection of muscimol into the MD caused a reduction in the local response to subiculum stimulation, which recovered after wash. (B) Average amplitude reduction and recovery with muscimol injection $(n=7)$. $P<0.01$ by paired t-test. 


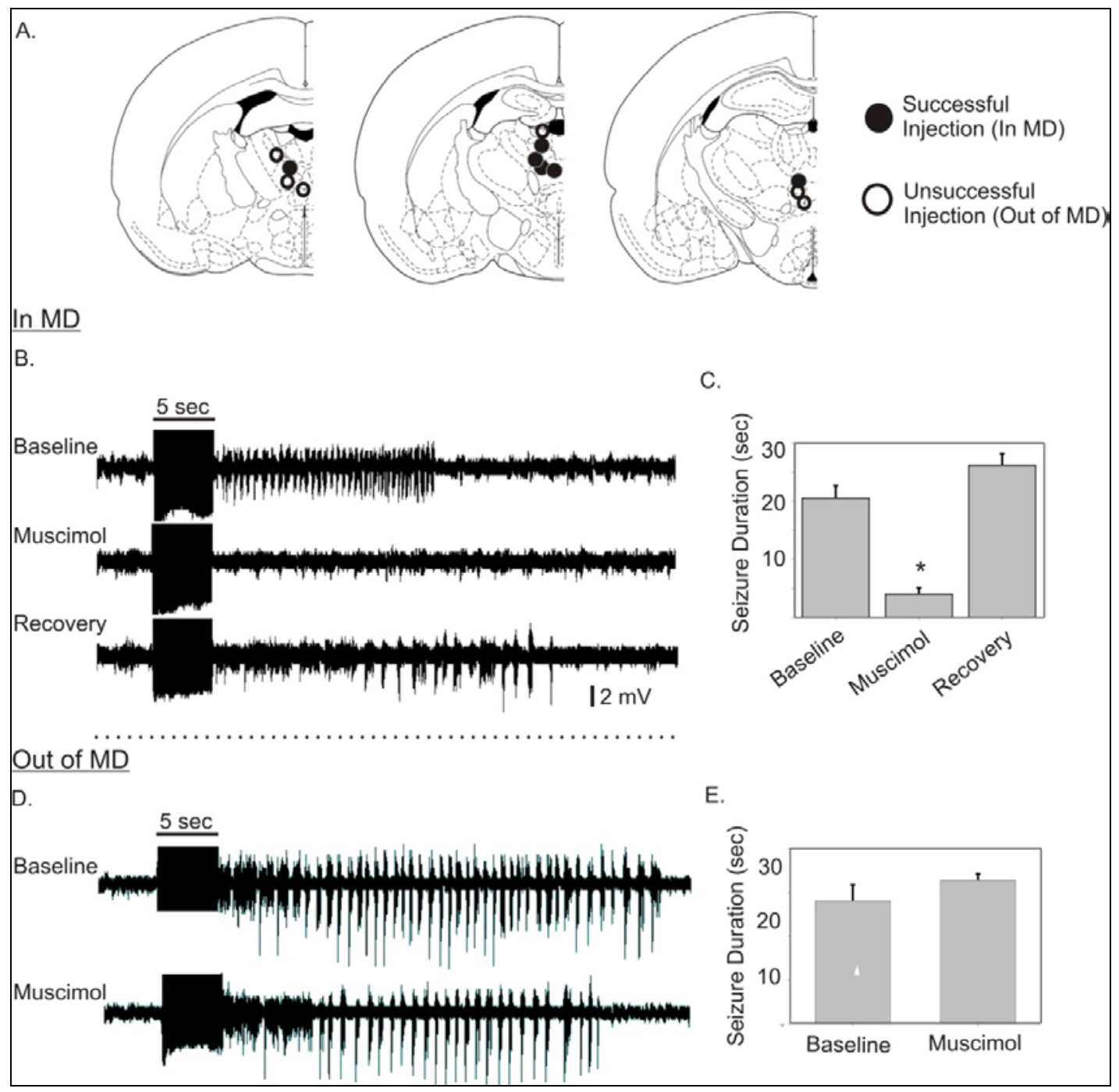

Figure 3: Effect of Muscimol injection into the MD on Seizures Generated in the Subiculum.

Injection of muscimol into the MD caused a reduction or ablation of seizures that were generated in the subiculum and recorded in the PFC. (A) Map of injection sites, including successful (seizure durations affected) and unsuccessful (no effect on seizures) injections. Note that successful injections were clustered in and around the MD. (B) Sample seizure showing reduction in seizure duration with muscimol application, and subsequent recovery. (C) Average seizure changes before, during and after a successful injection of muscimol in MD $(n=7)$, showing significant reduction in duration. $\mathrm{P}<0.05$ by paired t-test. (D) Sample unsuccessful injection, in which seizure duration did not change. (E) Average seizure durations before and after unsuccessful injections, showing no significant change $(n=5)$. 


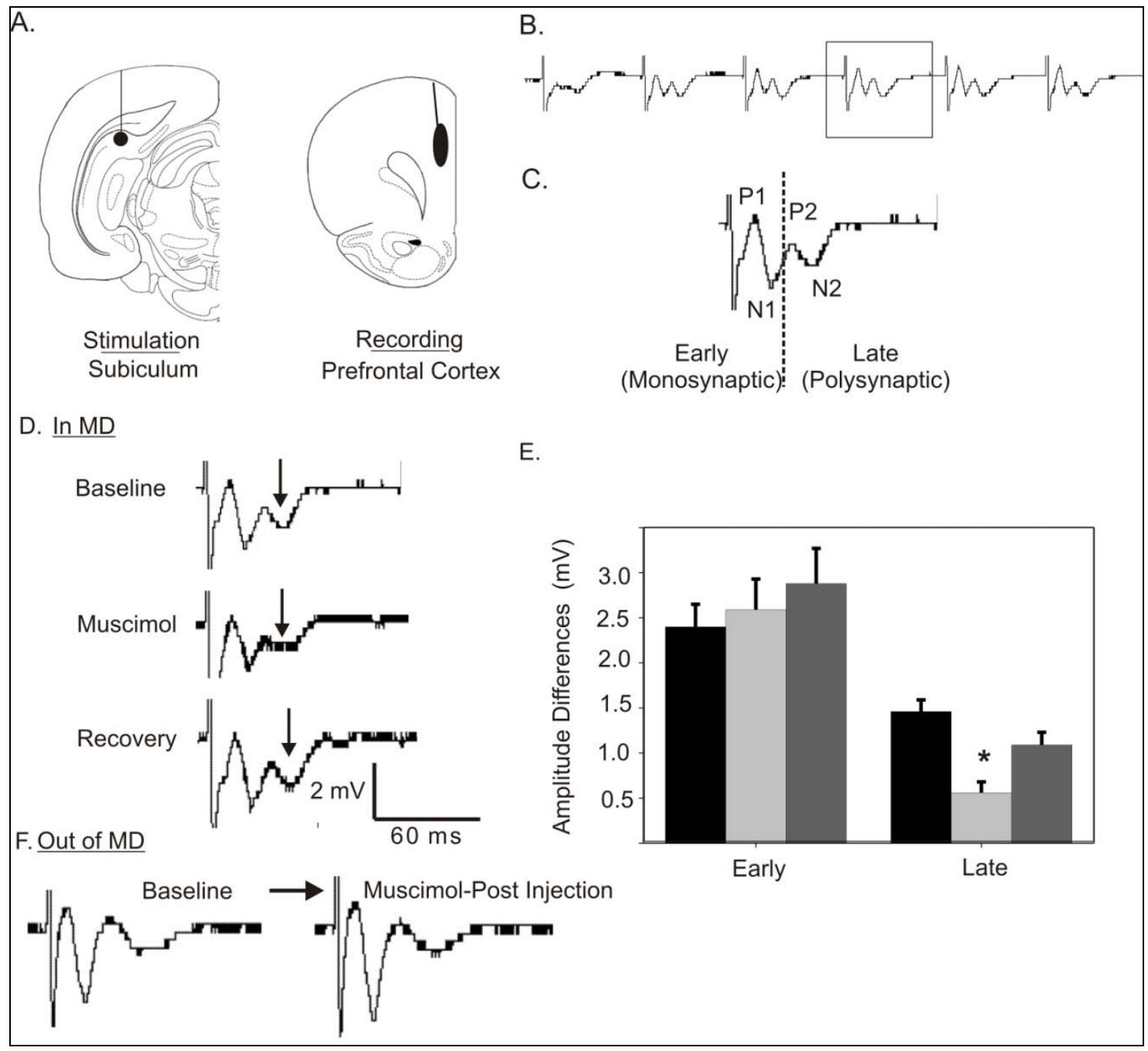

Figure 4: PFC Responses to Subiculum Stimulation Reduced By Injection of Muscimol into MD.

(A) Stimulation and recording sites for SB-PFC experiments. (B) Stimulation in a train yielded complex field responses in the medial PFC. (C) Average field latencies from polysynaptic circuit suggest a minimal thalamic influence on PFC responses to be approx. $35 \mathrm{~ms}$, suggesting that the "late" component of the response most likely contains influence from the thalamus (see Methods) (D) Injection of muscimol into the MD altered the late component of SB-PFC field potentials. (E) Graph of the differences between P1-N1(early) and P2-N2 (late). The early component did not change significantly, while the late peak was significantly reduced. $P<0.05$ by paired t-test. ( $F$ ) Sample responses from an injection outside of the MD, which did not affect seizures (see Figure 3). Note that there was no change in response amplitudes in either component. 


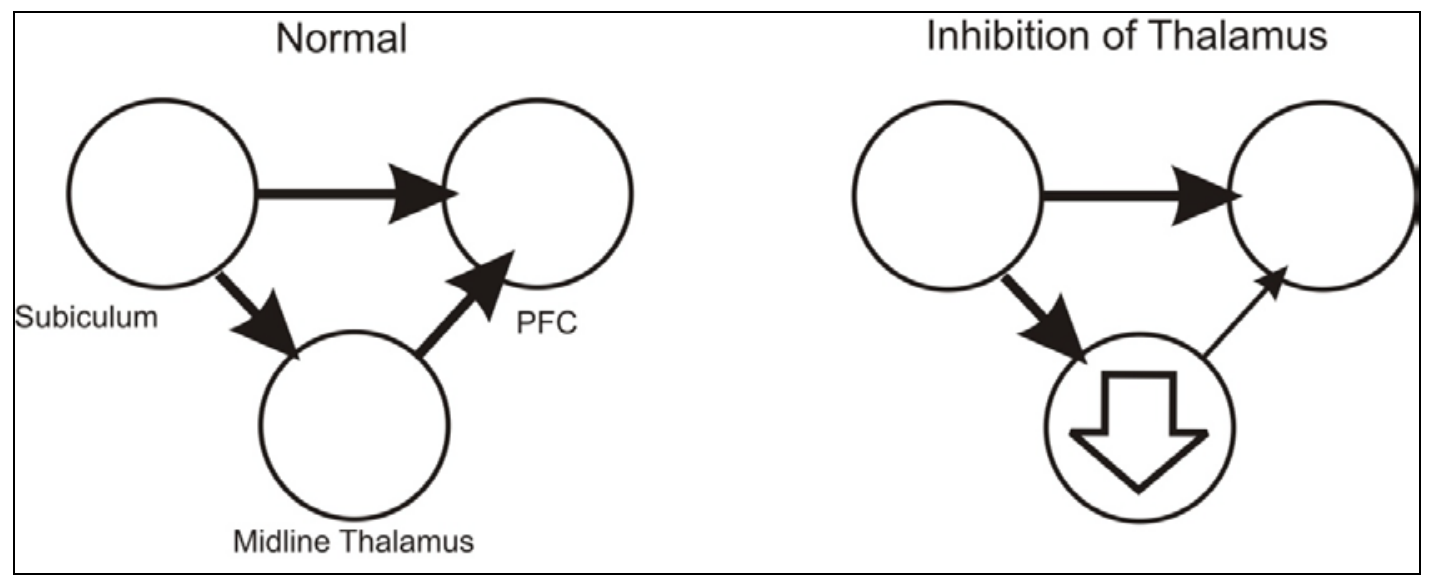

Figure 5: Model of Effect of Increased Inhibition in Midline Thalamus on SB-PFC signaling.

In the normal circuit, there is a direct monosynaptic connection between the hippocampus and the PFC. There is also a disynaptic connection that terminates in the thalamus before reconverging in the PFC. When the thalamic response is reduced, the thalamic output is weakened. This attenuates the functional role of the accessory excitatory thalamic output to the PFC, and also reduces the capacity for seizures to be generated in the SB-PFC pathway. 


\title{
Chapter V: Conclusions
}

\author{
Review of Findings
}

As stated in the Introduction, the work in this thesis had 3 main goals:

(1) Identify how signaling between the midline thalamus and a cortical target is altered in limbic epilepsy.

(2) Identify the role of the midline thalamus in limbic seizures.

(3) Identify the effect of modulation of the thalamus on distinct limbic circuits.

The results of the thesis, in Chapters II-IV, address these issues.

Chapter II showed that signaling between the midline thalamus and the PFC of the rat is altered in both kindled and chronic seizure models. It showed that the change in the chronic model could be attributed to the emergence of cell death in the thalamus, and not as a consequence of limbic seizures themselves. This work demonstrates that the effects of changes in the thalamus can extend beyond the limbic network and could facilitate spread to other circuits. It also suggested that behavioral abnormalities in animal models, and perhaps in human mTLE patients, could be partially attributed to altered thalamic output to regions such as the prefrontal cortex.

Chapter III showed that the midline thalamus is part of an excitatory signal amplification system through a divergent convergent circuitry with different limbic circuits. This work suggests that the midline thalamus facilitates seizure activity by prolonging depolarization at target structures, providing an additional excitatory drive during a seizure.

Chapter IV showed shows that GABAergic modulation of the mediodorsal nucleus causes a reduced responsiveness in thalamic neurons, which results in a diminished contribution to signaling and seizure spread in its divergent-convergent connections (specifically in the subiculum-prefrontal cortex pathway). Chapters III and 
IV further emphasize that the effect of the midline thalamus is very site specific, centering on the mediodorsal nucleus, since pharmacological manipulations of seizures outside of the mediodorsal region had little no effect.

\section{Role of the Midline Thalamus}

The findings in this thesis, in conjunction with previous studies, begin to suggest a model of how the midline thalamus influences limbic circuitry generally, and how that influence can translate into a role in seizure initiation and spread (Discussion Figure).

In the normal brain, the thalamus interacts with region-to-region signaling throughout the limbic network. It serves not only as a relay of information, but as an excitatory signal amplifier through multiple divergent-convergent pathways. This thalamic influence has a functional significance that may contribute to any number of limbic functions, including those involved in learning and memory.

In models of chronic seizures, evidence suggests that several things happen to the limbic network to create an operant seizure circuitry. In kindled seizure models, synapses are strengthened and become hypersensitive. The influence of the thalamus on other limbic circuits becomes more powerful. In chronic seizure models, cell death throughout the network causes reduced signaling, but surviving cells may form inappropriate connections in an attempt to compensate for the loss. The result is net hyperexcitability in the network. In the midline thalamus, cell loss throughout the nuclei impedes the ability of the thalamus to effectively interact with other circuits as it once did. However, the surviving thalamic cells become themselves hyperexcitable (Bertram et al. 2001), and would therefore still contribute to spreading and synchronizing seizure activity with their remaining connections.

Essentially, the theoretical role of the thalamus in seizures is two-fold: first, its normal, widespread connectivity ensures that any time the thalamus participates in seizures, the rest of the limbic network is affected by the seizure signal. Second, its influence as an amplifier allows it to help drive other structures over any threshold that would impede it from participating in seizure activity. In this way it acts as a distributor

and facilitator. This is the current working model of why the thalamus is so important to 


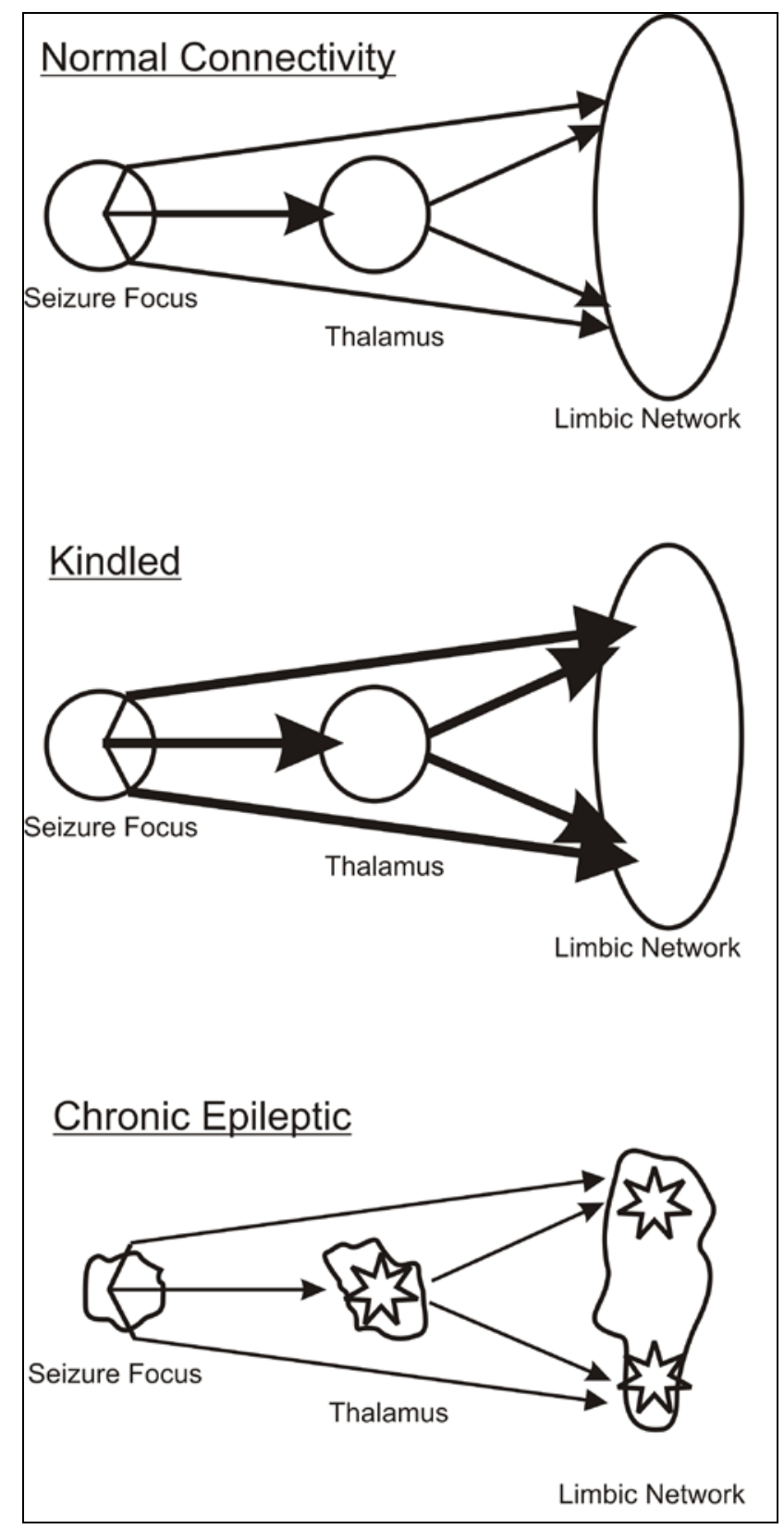

\section{Discussion Figure- Role of the Midline Thalamus in Limbic Seizures}

(Modified from Figure 8 of Chapter 3 ) In the normal connectivity of the midline thalamus and limbic network, the thalamus has direct connections with multiple limbic structures, and forms divergent-convergent circuits with distinct limbic pathways. In the kindled rat, synaptic strengthening allows all excitatory pathways to more easily facilitate seizure activity when overstimulated. In the chronic epileptic rat, much of the limbic network is damaged, and local changes cause hyperexcitability everywhere. Surviving cells maintain the connectivity of the network. Although signal strength is depleted, the network-wide excitability lowers the threshold for seizure activity enough that the existent thalamic circuitry is sufficient to facilitate seizures. 
limbic seizures, but there is clearly more work to elaborate on an clarify the details of this model.

The criticality of these thalamic roles — and they are critical—implies that the midline thalamus would make an important target for clinical interventions in mTLE patients. Based on this work, the effectiveness of such interventions would be based on several factors. First, these results show that the critical thalamic circuitry is very sitespecific; the mediodorsal should be targeted and hit in preliminary studies. Second, the functional importance of the thalamus, and its integrated role in normal limbic operations, suggests that it cannot be safely removed or lesioned without widespread consequences. Interventions should be as subtle as possible. Third, there are already ways of suppressing midline thalamic activity without external pharmacology (although it may be effective). There are many modulatory influences on the midline thalamus that may be targeted, as will be discussed.

\section{Future Research}

This thesis suggests several intriguing possibilities for further work.

First, the effect of thalamic inhibition/modulation on subiculum output to the PFC suggests that the thalamus has a large functional role in hippocampal signaling to the limbic network. A study could be undertaken to demonstrate the range and relative strength of thalamic influences on hippocampal targets. For example, field responses could be measured in other regions to which the hippocampus and midline thalamus both project, such as the amygdala and nucleus accumbens, and experiments similar to those carried out in Chapter 4 could be done. To find that the thalamus has a widespread influence on hippocampal outputs indirectly would be a powerful finding considering the tremendous role of the hippocampus on learning and memory.

Second, the study of thalamic modulation in Chapter 4 could be followed up by measuring the effect of stimulating structures that send GABAergic projections to the midline thalamus, thereby replicating, in a way, the effect of muscimol. As mentioned in the Discussion in Chapter 4, two such structures are the reticular nucleus of the thalamus, and certain regions of the ventral forebrain (specifically the globus pallidus, ventral 
pallidum and diagonal band.) Research into the effect of the reticular nucleus on the thalamus, and on seizures, is ongoing by other members of our lab. Work on these forebrain structures has not yet been done in limbic seizure models, although some conflicting but interesting results have been derived from other structures associated with the basal ganglia (Iadarola and Gale 1982, Bonhaus 1991, Shi et al. 2007). In some ways, the forebrain structures are more intriguing targets because they are bigger and easier to hit (although some experimentation would have to be done to find the most effective inhibitory region within and among those structures). If it is found that, clinically, the thalamus is not a logistically feasible target, perhaps stimulation of an auxiliary region is a possibility.

Third, the physiological relationship between neurons in different midline nuclei must be examined. Each nuclei sends overlapping projections throughout the network, and each nucleus receives afferents from many of the same sources. What is not known is if, and how, information is passed within and between the midline nuclei directly. Double retrograde labeling studies (including those done by the author) suggest that there are very few cells that project to more than one region. Rather, there seem to be discrete cells for each region spread throughout the nuclei. How those sparse groups interact with each other is unknown. A surprisingly small amount of published research has attempted to study relationships between thalamic neurons, and most of what has been done examines the relationship between certain nuclei and the reticular nucleus (Lozadi 1995, Lee et all 2010). The proximity of different nuclei may make such experiments logistically challenging, but if they were successful, they would provide a clearer understanding of how signals from the limbic network are processed through the thalamus.

Fourth, almost every circuit-based study in normal rats, including those in this thesis, must be re-considered in epileptic rats. As has been shown, the physiological dynamics of the limbic network change as the chronic model develops. The rules governing how the thalamus interacts with the network also change. There may be a resistance to certain drugs in epileptic animals, or a different effect, or a modified interaction downstream. A solid understanding of how the network operates normally is 
essential to understanding how seizures work, but that understanding falls short unless all the changes to that network in the seizure models are also understood and compared. With specific reference to this thesis, experiments demonstrating the influence of the thalamus in the SB-PFC and PC-EC pathways in the chronic epilepsy models could be undertaken.

It should also be noted that there are several avenues of research that have not been explored at all in this thesis, but which may have critical influences on the outcomes of the experiments recorded herein. For example the role of glia in the epileptic midline thalamus has not been examined, although there is evidence suggesting that alterations in both astrocytes and microglia may be important in epilepsy models (Fang et al. 2010, Yang et al. 2010). It is not known from the silver staining in Chapter 2, Figure 4, if the cell death observed was neuron-specific, or if local glia population numbers or interactions were altered. Furthermore, extracellular levels of neurotransmitters and ionic concentrations (glutamate, potassium, chloride, etc.) were not examined, although there is plenty of evidence for local chemical imbalances in the extracellular milieu also being important in seizure activity (Eid et al. 2008, Gorji et al. 2006). These factors may govern how the connectivity and physiological influence of the midline thalamus actually functions, and may complicate the model of seizure as discussed above, (although the fact that these factors were not examined does not change the accuracy or applicability of the experiments conducted in this thesis). The importance of future (and ongoing) studies of the cellular and molecular properties of thalamic cells, the extracellular thalamic space and thalamic targets in models of epilepsy should not be overlooked. 


\section{References}

Avoli M and Gloor P (1982) Interaction of cortex and thalamus in spike and wave discharges of feline generalized penicillin epilepsy. Exp Neurol 76: 196-217.

Barbas H, Haswell Henion TH, Dermon CR (1991) Diverse thalamic projections to the prefrontal cortex in the rhesus monkey. J Comp Neurol 313:65-94.

Barbas $\mathrm{H}$ (1995) Anatomic basis of cognitive-emotional interactions in the primate prefrontal cortex. Neurosci Biobehav Rev 19:499-510.

Bazhenov M, Timofeev I, Steriade M, Sejnowski TJ (1998) Cellular and network models for intrathalamic augmenting responses during10-Hz stimulation. J Neurophysiol 79:2730-2748.

Behrens TEJ, Johansen-Berg H, Woolrich MW, Smith SM, Wheeler-Kingshott CAM, Boulby PA, Barker GJ, Sillery EL, Sheehan K, Ciccarelli O, Thompson AJ, Brady JM and Mathews PM. (2003) Non-invasive mapping of connections between human thalamus and cortex using diffusion imaging. Nature Neuro 7: 750- 757.

Bell ML, Rao S, So EL, Trennery M, Kazemi N, Steed SM, Cascino G, Marsh R, Meyer FB, Watson RE, Giannini C and Worrell GA (2009) Epilepsy surgery outcomes in temporal lobe epilepsy with a normal MRI. Epilepsia 50: 2053-2060.

Ben-Ari Y, Tremblay E, Riche D, Ghilini G, Naquet R (1981) Electrographic,clinical and pathological alterations following systemic administration of kainic acid, bicuculline or pentrazole: metabolic mapping using deoxyglucose method with special reference to the pathology of epilepsy. Neuroscience 6:1361-1391.

Berendse HW, Groenwagen HJ (1991) Restricted cortical termination fields of the midline and interlaminar thalamic nuclei in the rat. Neuroscience 42:73-102.

Bertram EH (1997) Functional anatomy of spontaneous seizures in a rat model of epilepsy. Epilepsia 38:95-105.

Bertram EH, Cornett JF (1994) The evolution of a rat model of chronic spontaneous limbic seizures. Brain Res 661:157-162.

Bertram EH, Mangan PS, Zhang DX, Scott CA, Williamson JM (2001) The midline thalamus: alterations and a potential role in limbic epilepsy. Epilepsia 42:967-978.

Bertram EH, Scott C (2000). The pathalogical substrate of limbic epilepsy: neuronal loss in the medial dorsal thalamic nucleus as the consistent change. Epilepsia, 41: S3-S8.

Bertram EH, Williamson JM, Cornett JF (1997) Design and construction of a long-term continuous video-EEG monitoring unit for simultaneous recording of multiple small animals. Brain Res Protoc 2:85-97.

Bertram EH and Zhang DX (1999) Thalamic excitation of hippocampal CA1 neurons: a comparison with the effects of CA3 stimulation. Neuroscience 92: 15-26.

Bertram EH, Zhang DX, Mangan P, Fountain N and Rempe D (1998) Functional anatomy of limbic epilepsy: a proposal for central synchronization of a diffusely hyperexcitable network. Epilepsy Res 32: 194-205. 
Bertram EH, Zhang DX, Mangan P, Fountain N and Rempe D (1998). Functional anatomy of limbic epilepsy: a proposal for central synchronization of a diffusely hyperexcitable network. Epilepsy Res 32: 194-205.

Bertram EH, Zhang D, Williamson JM (2008) Multiple roles of midline dorsal thalamic nuclei in induction and spread of limbic seizures. Epilepsia 49:256-268.

Blackwood DHR, Kapoor V and Martin MJ (1981) Regional changes in cerebral glucose utilization associated with amygdaloid kindling and electroshock in the rat. Brain Res 224: 204-208.

Blumenfeld H, McNally KA, Vanderhill SD, Paige AL, Chung R, Davis K, Norden AD, Stokking R, Studholme C, Novotny EJ Jr, Zubal IG, Spencer SS.(2004) Positive and negative network correlations in temporal lobe epilepsy. Cereb Cortex 14: 892-902.

Bonhaus DW, Russell RD and McNamara JO (1991) Activation of substantia nigra pars reticulate neurons: role in the initiation and behavioral expression of kindled seizures. Brain Res 545: 4148.

Bonilha L, Rorden C, Castellano G, Cendes F, Li LM (2005) Voxel-based morphometry of the thalamus in patients with refractory medial temporal lobe epilepsy. Neuroimage 25: 1016-1021.

Brandt C, Potschka H, Loscher W, and Ebert U (2003) N-methyl-D-aspartate receptor blockade after status epilepticus protects against limbic brain damage but not against epilepsy in the kainate model of temporal lobe epilepsy. Neuroscience 118:727-740.

Carlson JD, Maire JJ, Martenson ME, Heinricher MM (2007) Sensitizing pain-modulating neurons in the ventral rostromedial medulla after peripheral nerve injury. J Neurosci 28:1322-13231.

Cassidy RM and Gale K (1998) Mediodorsal thalamus plays a critical role in the development of limbic motor seizures. J Neurosci 18:9002-9008.

Castro-Alamancos MA, Connors BW (1996) Short-term plasticity of a thalamocortical pathway dynamically modulated by behavioral state. Science $272: 274-277$.

Cavalheiro EA, Riche DA, Le Gal La Salle G (1982) Long-term effects of intrahippocampal kainic acid injection in rats: a method for inducing spontaneous recurrent seizures. Electroencephalogr Clin Neurophysiol 53:581-589.

Cavazos JE and Cross DJ (2006) The role of synaptic reorganization in mesial temporal lobe epilepsy. Epilep and Behav 8: 483-493.

Cavdar S, Onat FY, Cakmak YO, Yananli HR, Gulcebi M and Aker R (2008) The pathways connecting the hippocampal formation, the thalamic reuniens nucleus and the thalamic reticular nucleus in the rat. J Anat 212: 249-256.

Chapman CA and Racine R (1997) Converging inputs to the entorhinal cortex from the piriform cortex and medial septum: facilitation and current source density analysis. J Neurophys 78 : 2602-2615.

Churchill L, Zahm DS and Kalivas PW (1996) The mediodorsal nucleus of the thalamus in rats- I. Forebrain gabaergic innervation. Neuroscience 70: 93-102. 
Cornwall $\mathrm{J}$ and Phillipson OT (1988) Afferent projections to the dorsal thalamus of the rat as shown by retrograde lectic transport: I. The mediodorsal nucleus. Neuroscience 24: 10351049.

Cruikshank SJ, Urabe H, Nurmikko AV and Connors BW (2010) Pathway-specific feedforward circuits between thalamus and neocortex revealed by selective optical stimulation of axons. Neuron 65: 230-245.

Deacon RC, Penny C, Rawlins JN (2003) Effects of medial prefrontal cortex cytotoxic lesions in mice. Behav Brain Res 139:139-155.

Degenetais E, Thierry AM, Glowinski J and Gioanni Y (2003) Synaptic influence of hippocampus on pyramidal cells of the rat prefrontal cortex: an in vivo intracellular recording study. Cerebral Cortex 13: 782-792.

Dempsey EW, Morison RS. (1942) The electrical activity of a thalamocortical relay system. Am J Phys 138:283-291.

Deransart C, Riban V, Lê BT, Hechler V, Marescaux C, Depaulis A (1999) Evidence for the involvement of the pallidum in the modulation of seizures in a genetic model of absence epilepsy in the rat. Neurosci Lett. 265: 131-134.

Dickerson BC and Eichenbaum H (2010) The episodic memory system: neurocircuitry and disorders. Neuropsychopharmacology 35: 86-104

Dinocourt C, Petanjek Z, Freund TF, Ben-Ari Y, Esclapez M (2003) Loss of interneurons innervating pyramidal cell dendrites and axon initial segments in the CA1 region of the hippocampus following pilocarpine-induced seizures. J Comp Neurol 459:407-425.

Dolleman-Van der Weel MJ, Lopes de Silva FH and Witter MP (1997) Nucleus reuniens thalami modulates activity in hippocampal field CA1 through excitatory and inhibitory mechanisms. $J$ Neurosci 17: 5640-5650.

Eid T, Williamson A, Lee TS, Petroff OA, de Lanerolle NC (2008) Glutamate and astrocytes-key players in human mesial temporal lobe epilepsy? Epilepsia Suppl 2: 42-52.

Elger CE (2000) Semeiology of Temporal Lobe Seizures. Intractable Focal Epilepsy. Eds. Oxbury J, Polkey C and Duchowny M. Harcourt Publishers.

Engel J Jr. (1996) Introduction to temporal lobe epilepsy. Epilepsy Res 26:141-150.

Engel J Jr, Wolfson L and Brown L (1978) Anatomical correlates of electrical and behavioral events related to amygdaloid kindling. Ann Neurol 3: 538-544.

Fang M, Liu GW, Pan YM, Shen L, Li CS, Xi ZQ, Xiao F, Wang L, Chen D, Wang ZF (2010) Abnormal expression and spatiotemporal change of Slit2 in neurons and astrocytes in temporal lobe epileptic foci: A study of epileptic patients and experimental animals. Brain Res 1324: 1423.

Fisher R, (40 others) and the SANTE Study Group (2010) Electrical stimulation of the anterior nucleus of thalamus for treatment of refractory epilepsy. Epilepsia 51:899-908. 
Floresco SB and Grace AA (2003) Gating of hippocampal-evoked activity in prefrontal cortical neurons by inputs from the mediodorsal thalamus and ventral tegmental area. J Neurosci 23: 3930-3943.

Freedman LJ, Cassell MD (1991) Thalamic afferents of the rat infralimbic and lateral agranular cortices. Brain Res Bull 26:957-964.

Gabbott PLA, Warner TA, Jays PRL, Salway P, Busby SJ (2005) Prefrontal cortex in the rat: projections to subcortical autonomic, motor, and limbic centers. J Comp Neurol 492:145-177.

Gale K and Cassidy RM (1998) Mediodorsal thalamus plays a critical role in the development of limbic motor seizures. J Neurosci. 18: 9002-9009.

Garant DS, Xu SG, Sperber EF and Moshe SL (1993) The influence of thalamic GABA transmission on susceptibility of adult rats to flurothyl induced seizures. Epilepsy Res 15: 185192.

Garcia-Cabezas MA, Martinez-Sanchez P, Sanchez-Gonzalez MA, Garzon M, Cavada C (2009) Dopamine innervation in the thalamus: monkey versus rat. Cereb Cortex 19: 424-434.

Giguere M, Goldman-Rakic PS (1988) Mediodorsal nucleus: areal, laminar, and tangential distribution of afferents and efferents in the frontal lobe of rhesus monkeys. J Comp Neurol 277:195-213.

Glowinski H (1973) Cognitive deficits in temporal lobe epilepsy. J Nerv Ment Dis 157:120-137.

Goddard GV, McIntyre DC and Leech CK (1969) A permanent change in brain function resulting from daily electrical stimulation. Exp. Neurol. 25: 295-330.

Gorji A, Stemmer N, Rambeck B, Jurgens U, May T, Pannek HW, Behne F, Ebner A, Straub H, Speckmann EJ (2006) Neocortical environment in patients with intractable epilepsy: potassium and chloride concentrations. Epilepsia 47: 297- 310.

Guye M, Regis J, Tamura M, Wednling F, McGonical A, Chauvel P and Bartolomei F (2006) The role of corticothalamic coupling in human temporal lobe epilepsy. Brain 129: 1917-1928.

Hamam BN, Sinai M, Poirier G and Chapman CA (2007) Cholinergic suppression of excitatory synaptic responses in layer II of the medial entorhinal cortex. Hippocampus 17: 103-113.

Hanneson DK, Howland J, Pollock M, Mohapel P, Wallace AE, Corcoran ME (2001) Dorsal hippocampal kindling produces a selective and enduring disruption of hippocampally mediated behavior. J Neurosci 21:4443-4450.

Herkenham M (1978) The connections of the nucleus reuniens thalami: evidence for a direct thalamo-hippocampal pathway in the rat. J Comp Neuro 177: 589-610.

Herkenham M (1986) New perspectives on the organization and evolution of nonspecific thalamocortical projections. Cerebral Cortex. Jones EG, Peters A (Eds). Vol. 5. Platinum Publishers, New York, pp. 403-446.

Hoover WB, Vertes RP (2007) Anatomical analysis of afferent projections to the medial prefrontal cortex in the rat. Brain Struct Funct 212:149-179.

Hopkins A, Shorvon S and Cascino G, eds. (1995) Epilepsy. Pp 1-5. $2^{\text {nd }}$ Edition, Chapman and Hall. 
Hughes JR (2009). Absence seizures: a review of recent reports with new concepts. Ep and Behav 15:404-412.

Iadarola MJ, Gale K (1982) Substantia nigra: site of anticonvulsant activity mediated by gammaaminobutyric acid. Science 218: 1237-1240.

lizuka T, Sakai F, Suzuki N, Hata T, Tsukahara S, Fukuda M, Takiyama Y (2002) Neuronal hyperexcitability in stroke-like episodes of MELAS syndrome. Neurology 59:816-824.

Isaacson RI (2002) Limbic System. Encyclopedia of Life Sciences. Pps 1-4. John Wiley and Sons publishers. www.els.net.

Janszky J, Janszky I, Schulz R, Hoppe M, Behne F, Pannel HW and Ebner A (2005) Temporal Lobe Epilepsy with Hippocampal Sclerosis: Predictors for Long-Term Surgical Outcome. Brain 128: 395-404.

Jay TM and Witter MP (1991) Distribution of hippocampal CA1 and subicular efferents in the prefrontal cortex of the rat studied by means of anterograde transport of Phaseolus vulgarisleucoaglutinnin. J Comp Neuro 313: 574-586.

Jokeit H, Seitz RJ, Markowitsch HJ, Neumann N, Witte OW, Ebnar A (1997) Prefrontal asymmetric interictal glucose hypometabolism andcognitive impairment in patients with temporal lobe epilepsy. Brain 120:2283-2294.

Juhasz C, Nagy F, Watson C, da Silva EA, Muzik O, Chugani DC, Shah J, Chugani HT (1999) Glucose and [11C]flumazenil positron emission tomography abnormalities of thalamic nuclei in temporal lobe epilepsy. Neurology 53: 2037-2045.

Kawashima H, Yoshinori I, Grace AA, and Takita M (2006) Cooperativity between hippocampalprefrontal short term plasticity through associative long-term potentiation. Brain Res 1109: 3744.

Kerr KM, Agster KL, Furtak SC and Burwell RD (2007) Functional neuroanatomy of the parahippocampal region: the lateral and medial entorhinal areas. Hippocampus 17: 697-708.

Kim CH, Koo B-B, Chung CK, Lee J-M, Kim JS and Lee SK (2010) Thalamic changes in temporal lobe epilepsy with and without hippocampal sclerosis: a diffusion tensor imaging study. Epilepsy Res. (published online). doi:10.1016/j.eplepsyres.2010.03.002. 1-10.

Knopp A, Kivi A, Wozny C, Heinemann U and Behr J (2005) Cellular and network properties of the subiculum in the pilocarpine model of epilepsy. J Comp Neuro 482: 486-478.

Koyama S, Katayama Y, Maejima S, Hirayama T, Fujii M, Tsubokawa T (1993) Thalamic neuronal hyperactivity following transaction of the spinothalamic tract in the cat: involvement of N-methyl-D-aspartate receptor. Brain Res 612:345-350.

Kumar SS, Buckmaster PS (2006) Hyperexcitability, interneurons, and loss of GABAergic synapses in entorhinal cortex in a model of temporal lobe epilepsy. Neurobiol Dis 26:46134623.

Kwan P and Sperling MR (2009) Refractory seizures: try additional antiepileptic drugs (after two have failed) or go directly to early surgery evaluation? Epilepsia 50 (Suppl 8): 57-62. 
Lacroix L, Broerson LM, Weiner I, Feldon J (1998) The effects of excitotoxic lesions of the medial prefrontal cortex on latent inhibition, prepulse inhibition, food hoarding, elevated plus maze, active avoidance and locomotor activity in the rat. Neuroscience 82:431-442.

Laroche S, Jay TM and Thierry AM (1990) Long-term potentiation in the prefrontal cortex following stimulation of the hippocampal CA1/subicular region. Neurosci Letters 114: 184-190.

Lee S-C, Cruikshank SJ and Connors BW (2010) Electrical and chemical synapses between relay neurons in developing thalamus. J Physiol 588: 2403-2415.

Lehmann T-N, Gabriel S, Kovacs R, Eilers A, Kivi A, Schulze K, Lanksche WR, Meencke HJ, Heinemann $U$ (2000) Alterations of neuronal connectivity in area CA1 of hippocampal slices from temporal lobe epilepsy patients and from pilocarpine-treated epileptic rats. Epilepsia 41(Suppl. 6):S190-S194.

Lee RJ, Depaulis A, Lomax P and Olsen RW (1989) Anticonvulsant effect of muscimol injected into the thalamus of spontaneously epileptic Mongolian gerbils. Brain Res 487: 363-367.

Llano DA and Sherman SM (2009) Differences in intrinsic properties and local network connectivity of identified layer 5 and 6 adult mouse auditory cotricothalamic neurons support a dual corticothalamic projection hypothesis. Cerebral Cortex 19: 2810-2826

Lopez J, Wolff M, Lecourtier L, Cosquer B, Bontempi B, Dalrymple-Alford J and Cassel J-C (2009) The intralaminar thalamic nuclei contribute to remote spatial memory. J Neurosci 29:3302-3306.

Lothman EW, Bertram EH, Kapur J, Stringer JL (1990) Recurrent spontaneous hippocampal seizures in the rat as a chronic sequela to limbic status epilepticus. Epilepsy Res 6:110-118.

Lothman EW, Hatelid JM and Zorumski CF (1985) Functional mapping of limbic seizures originating in the hippocampus: a combined 2-deoxyglucose and electrophysiologic study. Brain Res 360: 92-100.

Lothman EW, Williamson JK (1993) Rapid kindling with recurrent hippocampal seizures: effect of stimulus frequency and train duration. Epilepsy Res 14:209-220.

Lozsadi DA (1995) Organization of connections between the thalamic reticular and anterior thalamic nuclei in the rat. $J$ Comp Neurol 358: 233-246.

Macrae CN, Moran JM, Heatherton TF, Banfield JF, Kelley WM (2004) Medial prefrontal activity predicts memory for self. Cereb Cortex 14:647-654.

Mangan PS and Bertram EH (1997) Shortened-duration GABA-A receptor-mediated synaptic potentials underlie enhanced CA1 excitability in a chronic model of temporal lobe epilepsy. Neuroscience 80: 1101-1111

Mangan PS and Bertram EH (1998) Ontogeny of altered synaptic function in a rat model of temporal lobe epilepsy. Brain Res 799:183-196.

Mangan PS, Scott CA, Williamson JM, Bertram EH (2000) Aberrant neuronal physiology in the basal nucleus of the amygdala in a model of chronic limbic epilepsy. Neuroscience 101:377391. 
Margerison JH, Corsellis JAN (1966) Epilepsy and the temporal lobes. A clinical, electroencephalographic and neuropathological study of the brain in epilepsy, with particular reference to the temporal lobes. Brain 89:499-530.

McFarland NR and Haber SN (2002) Thalamic relay nuclei of the basal ganglia form both reciprocal and nonreciprocal cortical connections, linking multiple frontal cortical areas. $J$ Neurosci 22: 8117-8132.

McIntyre DM and Gilby KL (2008) Mapping seizure pathways in the temporal lobe. Epilepsia 49 (supl. 3) 23-30.

McKenna JT and Vertes RP (2004) Afferent projections to nucleus reuniens of the thalamus. J Comp Neurol 480: 115-142.

Meeren HKM, Pijn JPM, van Lujtelaar ELJPM, Coenen AML, Lopes de Silva FH (2002) Cortical focus drives widewpread corticothalamic networks during spontaneous absence seizures in rats. J Neurosci 22: 1480-1495.

Meeren HKM, Veening JG, Moderscheim TAE, Coenen AML and van Luijtelaar G (2009) Thalamic lesions in a genetic rat model of absence epilepsy: dissociation between spike-wave discharges and sleep spindles. Exp Neurol 217: 25-37.

Mega MS, Cummings JL, Salloway S and Malloy P (1997) The limbic system: an anatomic, phylogenetic and clinical perspective. J Neuropsych 3:315-328.

Miller JW and Ferrendelli JA (1990) The central medial nucleus: thalamic site of seizure regulation. Brain Res 508: 297-300.

Mitchell AS, Baxter MG and Gaffan D (2007) Dissociable performance on scene learning and strategy implementation after lesions to magnocellular mediodorsal thalamic nucleus. $\mathrm{J}$ Neurosci. 27:11888-11895.

Mitelman SA, Brikman AM, Shihabuddin L, Newmark R, Chu KW, Buchsbaum SM (2005) Correlations between MRI-assessed volumes of the thalamus and cortical Brodmann's areas in schizophrenia. Schizophrenia Res 75:265-281.

Morel A, Magnin M and Jeanmonod D (1997) Multiarchitechtonic and stereotactic atlas of the human thalamus. J Comp Neurol 387: 588- 630.

Morimoto K, Fanestock M and Racine RJ (2004) Kindling and status epilepticus models of epilepsy: rewiring the brain. Neurobiology 73:1-60.

Morison RS and Dempsey EW (1943) Mechanism of thalamocortical augmentation and repetition. Am J Physiol 138:297-308.

Mueller SG, Laxer KD, Barakos J, Cheong I, Finlay D, Garcia P, Cardenas-Nicolson V and Weiner MW (2009) Involvement of the thalamocortical network in TLE with and without mesiotemporal sclerosis. Epilepsia. DOI 10.1111/j.1528-1167.2009.02413.x

Mulder AB, Arts MPM and Lopes de Silva FH (1997) Short and long-term plasticity of the hippocampus to nucleus accumbens and prefrontal cortex pathways in the rat, in vivo. Euro $\mathrm{J}$ Neuro 9: 1603-1611.

Nagaeva DV and Akhmadeev AV (2006) Structural organization, neurochemival charachteristics and connections of the reticular nucleus of the thalamus. Neuro and Behav Phys 36: 987-995. 
Namura S, Takada M, Kikuchi H, and Mizuno N (1994) Topographical organization of subicular neurons projecting to subcortical regions. Brain Res Bull 35: 221-231.

Nanobashvili Z, Chachua T, Nanobashvili A, Bilanishvili I, Lindvall O, and Kokaia Z (2003) Suppression of limbic motor seizures by electrical stimulation in thalamic reticular nucleus. Exp. Neurol. 181: 224-230.

Negyessy L, Goldman-Rakic PS. (2005) Morphometric characterization of synapses in the primate prefrontal cortex formed by afferents from the mediodorsal thalamic nucleus. Exp Brain Res 164:148-154.

Patel S, Millan MH and Meldrum BS (1988) Decrease in excitatory transmission within the lateral habenula and the mediodorsal thalamus protects against limbic seizures in rats. Exp Neurol 101: 63-74.

Paxinos G, Watson C. (1998) The rat brain in stereotaxic coordinates. 4th ed. Academic Press, San Diego, CA.

Pellegrini A, Musgrave J and Gloor P (1979) Role of afferent input of subcortical origin in the genesis of bilaterally synchronous epileptic discharges of feline generalized penicillin epilepsy. Exp. Neurol 64: 155-173.

Powell DA and Churchwell J (2002) Mediodorsal thalamic lesions impair trace eyeblink conditioning in the rabbit. Learning and Memory 9:10-17.

Price JL. (2005) Free will versus survival: brain systems that underlie intrinsic constraints on behavior. J Comp Neurol 493:132-139.

Racine RJ, Adams B, Osehobo P, Fahnestock M (2002) Neural growth, neural damage and neurotrophins in the kindling model of epilepsy. Adv Exp Med Biol 497:149-170.

Ray JP, Russchen FT, Fuller TA and Price JL (1992) Sources of presemuptive glutamatergic/apartic afferents to the mediodorsal nucleus of the thalamus in the rat. $\mathrm{J}$ Comp Neurol 32: 435-456.

Reichova I and Sherman SM (2004) Somatosensory corticothalmic projections: distinguishing drivers from modulators. J Neurophysiol 92: 2185-2197.

Represa A, Jorquera I, La Salle GLG, Yehezkel B-A (1993) Epilepsy induced collateral sprouting of hippocampal mossy fibers: does it induce the development of ectopic synapses with granule cell dendrites? Hippocampus 3:257-268.

Saddiqui AH and Joseph SA (2005) CA3 axonal sprouting in kainite-induced chronic epilepsy. Brain Res 1066: 129-146.

Schlag JD, Chaillet F (1962) Thalamic mechanisms involved in cortical desynchronization and recruiting responses. Electroencephalogr Clin Neurophysiol 15:39-62.

Shi L-H, Luo F, Woodward DJ, McIntyre DC and Chang J-Y (2007) Temporal sequence of ictal discharges propogation in the corticolimbic basal ganglia system during anygdala kindled seizures in freely moving rats. Epilepsy Res 73: 85-97. 
Sloan DM and Bertram EH. (2009) Changes in midline thalamic recruiting responses in the prefrontal cortex of the rat during the development of chronic limbic seizures. Epilepsia 50:556565.

Sloviter RS, Zappone CA, Harvey BD, Frotscher M. (2006) Kainic acid induced recurrent mossy fiber innervation of dentate gyrus inhibitory interneurons: possible anatomical substrate of granule cell hyperinhibition in chronically epileptic rats. J Comp Neurol 494:944-960.

So NK (1991) Depth electrode studies in mesial temporal epilepsy. Epilepsy Surgery. Luders J ed. Raven Press. New York. 371-384.

Steriade M, Domich L, Oakson G and Deschenes M (1987) The deafferented reticular thalamic nucleus generates spindle rhythmicity. J Neurophys 57: 260-272.

Sun C, Mtchedlishvili Z, Bertram EH, Erisir A, Kapur J (2007) Selective loss of dentate hilar interneurons contributes to reduced synaptic inhibition of granule cells in an electrical stimulation-based animal modelof temporal lobe epilepsy. J Neurophysiol 500:876-893.

Swanson LW (1981) A direct projection from ammon's horn to prefrontal cortex in the rat. Brain Res. 217: 150-154.

Takaya S, Hanakawa T, Hashikawa K, Ikeda A, Sawamoto N, Nagamine T, Ishizu K, Fukuyama $\mathrm{H}$. (2006) Prefrontal hypofunction in patients with intractable mesial temporal lobe epilepsy. Neurology 67:1674-1676.

Theyel BB, Llano DA and Sherman SM (2010) The corticothalamocortical circuit drives higherorder cortex in the mouse. Nature Neuro 13: 84-88.

Turski L, Cavalheiro EA, Sieklucka-Dziuba M, Ikonomidou-Turski C. Czuczwar SJ, Tusrski WA (1986) Seizures produced by pilocarpine: neuropathological sequelae and activity of glutamate decarboxylase in the rat forebrain. Brain Res. 398: 37-48.

Van der Werf YD, Witter MP, Groenwagen HJ. (2002) The intralaminar and midline nuclei of the thalamus: anatomical functional evidence for participation in processes of arousal and awareness. Brain Res Rev 39:107-140.

Van Der Werf YD, Jolles J, Witter MP and Uylings HBM (2003) Contributiions of thalamic nuclei to declarative memory functioning. Cortex 39: 1047-1062.

Van Elst LT, Krishnamoorthy ES, Baumer D, Selai C, von Gunten A, Gene-Cos N, Ebert D, and Trimble MR (2003) Psychopathalogical profile in patients with severe bilateral hippocampal atrophy and temporal lobe epilepsy: evidence in support of the Geschwind syndrome? Epilepsy Behav. 4: 291- 297

Vertes RP (2004) Differential projections of the infralimbic and prelimbic cortex in the rat. Synapse 51:32-58.

Vertes RP, Linley SB and Hoover WB (2010) Pattern of distribution of seratonergic fibers to the thalamus of the rat. Brain Struct Funct. E-pub ahead of print DOI 10.1007/s00429-010-0249$\mathrm{x}$.

Verzeano M, Lindsley DB, Magoun HW (1953) Nature of recruiting response. J Neurophysiol 16:183-195. 
Viisanen H, Perrovaara A (2007) Influence of peripheral nerve injury on response properties of locus coeruleus neurons and coeruleospinal antinociception in the rat. Neuroscience 146:1785-1794.

Viskontas IV, McAndrews MP, Moscovitch M (2000) Remote episodic memory deficits in patients with unilateral temporal lobe epilepsy and excisions. J Neurosci 20:5853-5857.

Vogt BA, Hof PR, Friedman DP, Sikes RW and Vogt LJ (2008) Norepinephrinergic afferents and cytology of the macaque monkey midline, mediodorsal, and intralaminar thalamic nuclei. Brain Struct Funct 21: 465- 479.

Voltzenlogel V, Despres O, Vignal J-P, Steinhoff BJ, Kehrli P, Manning L (2006) Remote memory in temporal lobe epilepsy. Epilepsia 47:1329-1336.

Weibe S, Blume WT, Girvin JP, Eliasziw M (2001) A randomized, control trial for surgery of temporal lobe epilepsy. New Engl J Med 345: 311-318.

Yang F, Liu ZR, Chen J, Zhang SJ, Quan QY, Huang YG, Jiang W (2010) Roles of astrocytes and microglia in seizure-induced aberrant neurogenesis in the hippocampus of adult rats. $J$ Neurosci Res 88: 519-529.

Yilmazer-Hanke DM, Wolf HK, Schramm J, Elger CE, Weistler OD,Blumcke I (2000) Subregional pathology of the amygdala complex and entorhinal region in surgical specimens from patients with pharmacoresistant temporal lobe epilepsy. J Neuropathol Exp Neurol 59:907-920.

Zhang DX and Bertram EH (2002) Midline thalamic region: widespread excitatory input to the entorhinal cortex and amygdala. J Neurosci 22:3277-3284. 